\title{
Cosmological solutions, a new wick-rotation, and the first law of thermodynamics
}

\author{
J. Gutowski, ${ }^{a}$ T. Mohaupt ${ }^{b}$ and G. Pope ${ }^{b}$ \\ ${ }^{a}$ Department of Mathematics, University of Surrey, \\ Guildford, GU2 7XH, U.K. \\ ${ }^{b}$ Department of Mathematical Sciences, University of Liverpool, \\ Liverpool, L69 7ZL, U.K. \\ E-mail: J.Gutowski@surrey.ac.uk, Thomas.Mohaupt@liv.ac.uk, \\ Giacomo@liv.ac.uk
}

ABSTRACT: We present a modified implementation of the Euclidean action formalism suitable for studying the thermodynamics of a class of cosmological solutions containing Killing horizons. To obtain a real metric of definite signature, we perform a "triple Wick-rotation" by analytically continuing all spacelike directions. The resulting Euclidean geometry is used to calculate the Euclidean on-shell action, which defines a thermodynamic potential. We show that for the vacuum de Sitter solution, planar solutions of Einstein-Maxwell theory and a previously found class of cosmological solutions of $\mathcal{N}=2$ supergravity, this thermodynamic potential can be used to define an internal energy which obeys the first law of thermodynamics. Our approach is complementary to, but consistent with the isolated horizon formalism. For planar Einstein-Maxwell solutions, we find dual solutions in Einstein-anti-Maxwell theory where the sign of the Maxwell term is reversed. These solutions are planar black holes, rather than cosmological solutions, but give rise, upon a standard Wick-rotation to the same Euclidean action and thermodynamic relations.

Keywords: Black Holes in String Theory, p-branes, Supergravity Models

ARXIV EPRINT: 2008.06929 


\section{Contents}

1 Introduction and motivation 1

2 Euclidean action formalism $\quad 5$

2.1 Gravitational and thermodynamic partition functions 5

2.2 Simple Wick-rotation 6

2.3 Triple Wick-rotation 8

$\begin{array}{lll}2.4 & \text { Surface gravity and temperature } & 10\end{array}$

3 Thermodynamics of the de Sitter solution $\quad 14$

$\begin{array}{lll}3.1 & \text { Static patch, single Wick-rotation } & 14\end{array}$

$\begin{array}{lll}3.2 & \text { Dynamic patch, triple Wick-rotation } & 16\end{array}$

4 Planar solutions to the Einstein-Maxwell theory 19

$\begin{array}{lll}4.1 \text { Static patch } & 19\end{array}$

$\begin{array}{ll}4.2 & \text { Dynamic patch } \\ & 20\end{array}$

$\begin{array}{lll}4.3 & \text { Euclidean action } & 21\end{array}$

5 Planar solutions to the STU model $\quad 24$

5.1 Dynamic patch 25

$\begin{array}{ll}5.2 \text { Euclidean action } & 28\end{array}$

6 Comparison to the isolated horizon formalism 32

6.1 Planar solutions of the STU model $\quad 32$

6.2 Planar solutions to Einstein-Maxwell theory 36

$\begin{array}{lll}7 & \text { Planar solutions to Einstein-anti-Maxwell theory } & 37\end{array}$

$\begin{array}{lll}7.1 \text { The solution } & 38\end{array}$

$\begin{array}{lll}7.2 & \text { Euclidean action } & 39\end{array}$

8 Discussion and outlook $\quad 42$

$\begin{array}{ll}\text { A Conventions } & 44\end{array}$

$\begin{array}{ll}\text { B Extrinsic curvature } & 46\end{array}$

$\begin{array}{ll}\text { C Charges and Hodge dualisation } & 48\end{array}$

D Kruskal extensions and classification of trapping horizons $\quad 50$

$\begin{array}{lll}\text { D.1 Black hole solutions } & 51\end{array}$

D.1.1 Defining Kruskal coordinates $\quad 52$

D.1.2 Calculation of expansions $\quad 55$

$\begin{array}{lll}\text { D.2 Cosmological solutions } & 58\end{array}$ 
D.2.1 Defining Kruskal coordinates $\quad 58$

$\begin{array}{ll}\text { D.2.2 Calculating the expansions } & 60\end{array}$

$\begin{array}{ll}\text { D.3 Eddington-Finkelstein coordinates } & 61\end{array}$

D.4 Asymptotic limits of planar Reissner-Nordström-like solutions 64

E Grand canonical ensemble (with fixed volume) 65

\section{Introduction and motivation}

The laws of black hole mechanics [1] were initially thought of as formal analogies of the laws of thermodynamics, but subsequent work has shown that they have a genuine thermodynamical interpretation. This suggests that the relation between classical and quantum gravity may be analogous to the relation between thermodynamics and statistical mechanics $[2-5]$. This is among the most compelling clues that we have about the nature of quantum gravity. It is therefore important to identify ever-larger classes of solutions to classical gravity which obey the laws of black hole mechanics, or variant versions thereof.

One setting in which the laws of black hole mechanics can be derived is static, asymptotically flat spacetimes containing a Killing horizon. ${ }^{1}$ Then the first law of black hole mechanics takes the form

$$
d M=\frac{\kappa}{8 \pi G} d A+\mu_{i} d \mathcal{Q}^{i}
$$

where $M$ is the mass, $\kappa$ is the surface gravity, $A$ is the area of the black hole horizon and $\mathcal{Q}^{i}, \mu_{i}$ are a set of conserved charges and their associated potentials. This statement does not involve any thermodynamics and is derived using geometrical reasoning. Yet, the seminal work by Bekenstein [2] and Hawking [3] has demonstrated that this relation can be interpreted as the first law of thermodynamics through identifying

$$
S=\frac{A}{4 G}, \quad T_{H}=\frac{\kappa}{2 \pi},
$$

where $S$ is the entropy and $T_{H}$ is the Hawking temperature. With these identifications, the first law of black hole mechanics becomes the first law of thermodynamics:

$$
d E=T_{H} d S+\mu_{i} d \mathcal{Q}^{i} .
$$

Here the internal energy $E$ is understood to be equal to the mass of the black hole solution, while the charges $\mathcal{Q}^{i}$ replace the particle numbers of a grand ensemble, as usual in relativistic thermodynamics.

One obstruction in generalising this statement to Killing horizons in more general spacetimes - in particular, those which are not asymptotically flat and not static — is

\footnotetext{
${ }^{1}$ This generalizes to the larger class of stationary spacetimes, where the first law also contains a term involving the angular momentum and rotation velocity of the spacetime. But since the solutions which we will consider in this paper are of the more restricted, static type, we will neglect this term from the beginning.
} 
the definition of the mass $M$, which takes the role of energy $E$. Diffeomorphism invariance prevents one from assigning a total momentum four-vector, and hence a mass to regions of spacetime in general. For asymptotically flat spacetimes the ADM construction can be used to define a total mass [6]. For Killing horizons with an asymptotically flat static region, this is equivalent to the Komar construction [7], where the mass is a conserved charge associated with a timelike Killing vector which becomes null on the horizon. In these constructions, the normalisation of the mass is implied by the 'natural' normalisation of this Killing vector field, which is that the Killing vector field has unit norm at infinity. Mass-like quantities can be defined in more general situations. For example, the quasi-local mass of Brown and York [8] is well-defined when the region is stationary, though the resulting mass parameter is necessarily position-dependent and thus does not have a natural normalisation. Wald's formalism $[9,10]$ allows one to associate conserved charges to closed surfaces in general diffeomorphism invariant theories of gravity, and provides a setting for deriving the first law. For space-times which are locally asymptotic to AdS spaces, conserved charges and thermodynamics can be defined using the variational principle and holographic renormalization, see [11] and references therein. In [12] it was stressed that the essential requirement to formulate black hole thermodynamics is to have a consistent variational principle, which then automatically takes care of the finiteness of conserved charges. This approach is not limited to boundary conditions which are locally asymptotically AdS. In particular, it was applied [12] to the STU-model of four-dimensional $\mathcal{N}=2$ supergravity with conical boundary conditions. Further work which applies variational principles to spacetimes with AdS asymptotics includes [13-15].

There also are approaches which formulate black hole thermodynamics strictly in terms of near horizon data. Where a comparison to approaches with asymptotic boundary conditions is possible, they agree up to an ambiguity associated with the normalization of the horizontal Killing vector field. One such approach is Ashtekar's isolated horizon formalism [16], which recasts the first law using only quantities defined locally on the horizon. This includes a mass-like parameter whose definition and normalization is fixed by imposing that the first law takes its standard form. More recently, there has been much interest in the near horizon behaviour of near-extremal black holes, in the context of studying nonintegrable conserved charges and scalar hair. In these approaches one uses the existence of an asymptotic $A d S_{2}$ factor to perform a reduction to two dimensions. The reduced system is largely determined by its symmetries, and related to the JT and SYK models, see for example [17-23].

In the present paper we study a class of spacetimes with Killing horizons, which has a causal structure complementary to black holes in the sense that the exterior region is non-static and asymptotic to a Kasner cosmology in the infinite past and future, while the interior region is static and terminates in a timelike curvature singularity. These spacetimes, which are solutions to $\mathcal{N}=2$ supergravity with vector multiplets, have planar symmetry and were found in [24], when attempting to generalise the black brane type solutions of [25]. The simplest member of this family, which is obtained by imposing that all scalar fields are constant, is the planar version of the Reissner-Nordström solution of Einstein-Maxwell theory. As explained in [24], the planar symmetry has the effect of preventing the existence 
of the static asymptotically flat regions familiar from the spherically symmetric ReissnerNordström solution. One is left with a dynamical region which is now the outer part, and a static inner region around the singularity. The resulting conformal diagram is that of a maximally extended Schwarzschild spacetime, rotated by 90 degrees, or equivalently, the conformal diagram of a spherical Reissner-Nordström solution with the asymptotically flat regions (called 'type I' in most references) removed, see figure 2.

While this situation is complementary to the usual set-up of black hole thermodynamics, we will show that it is nevertheless possible to define a mass-like quantity $E$ and to derive a relation which takes the form of the first law of thermodynamics, as well as a Smarr relation, with $E$ playing the role of energy. Since the static region terminates in a singularity, there are two options we will explore: the first is to work with the exterior, nonstatic region, the second is to use the isolated horizon formalism which only requires local near-horizon data. We find that both approaches lead to mutually consistent results. Most of the paper is devoted to adapting the Euclidean action formalism to the dynamic patch (non-static region). While applying the isolated horizon formalism is more straightforward, we will see that the Euclidean action formalism provides us with additional insights. In particular we obtain a thermal partition function, and we will discover an interesting 'duality' between the thermodynamics of cosmological solutions and the thermodynamics of planar black holes in theories where the sign of the Maxwell term has been flipped. ${ }^{2}$

Our adaptation of the Euclidean action approach works as follows. In the standard setting based on a static exterior region with a well behaved asymptotic boundary, one Wick-rotates the time coordinate and obtains a smooth, positive definite metric on a real slice of the complexification of the original solution. One then substitutes the Euclideanised solution into the action, thus obtaining a function which depends on the parameters of the solution. In this step of the procedure, boundary terms play a central role. The exponential of the resulting Euclidean action can be viewed as the saddle point approximation to the full Euclidean functional integral. Following Gibbons and Hawking [4] we can interpret this expression as a thermal partition function. Given this, the energy $E$ can be computed by taking suitable derivatives with respect to combinations of parameters, of the solution which correspond to thermodynamic variables. By then computing its variation $\delta E$, one can check whether the first law is satisfied. For the type of solution we are interested in, the singularities in the static region prevent us from computing the Euclidean action in the static patch, as its boundary is a singularity. Therefore, we work instead in the dynamic outer region, as it is well behaved at its asymptotic boundary, located at past timelike infinity. ${ }^{3}$ Since the horizontal Killing vector field is spacelike in this region, we cannot apply a Wick-rotation in time, which would make spacetime complex. Instead, we perform a triple Wick-rotation in all spacelike coordinates, which provides a real slice of the complexified spacetime with a (negative) definite metric. Using this slice, we obtain a well behaved Euclidean action. We note that the standard argument for identifying the

\footnotetext{
${ }^{2}$ Such sign flips appear in type-II* string theories, as we will discuss in section 8 .

${ }^{3}$ The global solutions have a second cosmological region which is related to this region by time-reversal, see figure 2. For thermodynamics we choose pairs of patches such that the exterior and interior are related by future-pointing null rays, that is regions III/IV or III/I.
} 
resulting Euclidean action with a thermodynamic potential depends on the Killing vector being timelike, and thus being related to time translations and energy. In the dynamic outer patch, the Killing vector is spacelike and thus corresponds to spatial translations and momentum. We proceed formally and relate our Euclidean action to a thermodynamic potential, leaving questions about the underlying microscopic theory aside. The 'energy' $E$ is defined as a derivative of this potential, and we prove that its variation $\delta E$ satisfies a relation which takes the exact form of the first law. As a further consistency check, we also apply the isolated horizon formalism, which imposes the first law and this way obtains an expression for the energy, and we find that the results of both formalisms agree.

The structure of the paper is as follows. We begin by introducing the Euclidean action formalism and reviewing the relevant ingredients. After an overview of standard techniques, the procedure of the triple Wick-rotation is defined. Following this, three examples of the triple Wick-rotation are given. First, the de Sitter solution is discussed in section 3, serving as a simple example of the first law for a vacuum solution, where we can compare against a standard Wick rotation in the static region as a consistency check. Then, in section 4 , the planar Reissner-Nordström solution to the Einstein-Maxwell system is studied using the triple Wick-rotation. A thermodynamic potential is derived, and from this, the first law is verified. This solution can be regarded as a limit of a family of solutions to the STU model of $\mathcal{N}=2$ supergravity, for which we verify the first law in section 5 . The method is applied again for the full STU model, allowing the definition of a mass-like parameter which, when varied, gives the first law. In the following section 6 , these results are supported through an alternative calculation using the isolated horizon technique of [16]. In section 7 we find a dual planar Reissner-Nordström solution in Einstein-anti-Maxwell theory, that is, in a theory where the sign of the Maxwell term is flipped. In this solution, the roles of the interior and exterior region are exchanged, and so the solution is a planar black hole with a static exterior region. This allows us to apply a standard Wick rotation, and we find that this solution has the same Euclidean action and thermodynamic relations as the planar ReissnerNordström solution. While we do not discuss the embedding of these solutions into string theory, we point out that this duality between solutions, as well as their connection through a common Euclidean section, is related to the existence of a 'twisted' version of the $\mathcal{N}=2$ supersymmetry algebra, and to timelike T-duality [26, 27]. We conclude with a physical interpretation and discussion of the work completed in this paper in section 8. Some of the calculational details are relegated to the appendices, together with a summary of the conventions used in this work. Specifically, appendix A summarises our conventions, while appendix $\mathrm{B}$ reviews extrinsic curvature, to the extent that is needed to compute boundary terms for the gravitational action. Appendix $\mathrm{C}$ reviews the definition and normalization of charges, and gives details of the dualization of magnetic to electric charges that we use in the main part. The quite substantial appendix D presents details of the maximal analytical extensions for all solutions considered plus the Schwarzschild solution for reference. This includes the definition of Kruskal and of advanced and retarded Eddington-Finkelstein coordinates, the computation of the expansion of null congruences, and the classification of horizons. We also show how type A-III vacuum Einstein solutions arise as asymptotic limits, and we show that the maximally extended planar Reissner-Nordström solution is a 
bouncing cosmology, which interpolates between, and regularizes, two Kasner cosmological solutions. Appendix E collects some thermodynamic relations for reference.

\section{Euclidean action formalism}

In this section, we first review the standard Euclidean action formalism, which interprets the saddle point approximation of the partition function for a gravitational theory as a thermodynamic partition function $[4,5]$. Then we present a modification which assigns a Euclidean action to a dynamic ${ }^{4}$ spacetime by using a triple Wick-rotation.

\subsection{Gravitational and thermodynamic partition functions}

The thermodynamic canonical partition function $Z(\beta)$ for a system with a Hamiltonian $\hat{H}$ is defined by

$$
Z(\beta):=e^{-\beta F}=\operatorname{Tr} e^{-\beta \hat{H}},
$$

where $F$ is the free energy and $\beta$ is the inverse temperature. For a system with a conserved charge $\mathcal{Q}$, the thermodynamic potential depends on the conserved charge in addition to its dependence on temperature, $F=F(\beta, \mathcal{Q})$. The grand canonical ensemble is defined by keeping the charge constant and letting the corresponding intensive thermodynamic variable, the chemical potential $\mu$, fluctuate. The corresponding thermodynamic partition function is the grand canonical partition function:

$$
\mathcal{Z}(\beta, \mu):=e^{-\beta \Omega}=\operatorname{Tr} e^{-\beta \hat{H}},
$$

where $\Omega(\beta, \mu)$ is the grand potential. Note that we are suppressing the contribution of a pressure/volume term usually seen in the thermodynamic potentials. From a gravitational perspective, these arise from rotations and angular momentum, or, in the case of planar solutions, translations and linear momentum, which are not present in the solutions we consider in this paper. The thermodynamic relations for such an ensemble are summarized in appendix $\mathrm{E}$ for convenience.

To illustrate the correspondence between partition functions of quantum (field) theories and thermodynamic partition functions, we consider the case of a quantum particle. The time-evolution operator admits a path integral representation involving the classical action

$$
\left\langle x\left|e^{-i t H}\right| x^{\prime}\right\rangle=\int \mathcal{D} x e^{i S[x]},
$$

where we have set $\hbar=1$. By Wick-rotating the time coordinate $t \rightarrow-i \beta$ and taking the trace, which in the path integral corresponds to integrating over paths periodic in time, one obtains

$$
\operatorname{Tr} e^{-\beta H}=\int \mathcal{D} x e^{-S_{E}[x]}=e^{-\beta F},
$$

where $\beta$ is interpreted as inverse temperature, and where $F$ is the free energy.

\footnotetext{
${ }^{4}$ Here and in the following 'dynamic' means 'non-stationary', that is a spacetime without a timelike Killing vector field.
} 
It is straightforward, at least at a formal level, to extend this prescription to quantum field theories. In a quantum theory including gravity, the path integral is performed over the space of all metrics $g$, as well as over the matter fields $\varphi$,

$$
Z=\int \mathcal{D} g \mathcal{D} \varphi e^{-S_{E}[g, \varphi]}
$$

While it is challenging to give a precise meaning to the full path integral, one can proceed formally and attempt to make sense of it in a saddle point approximation. This leads to the expression $Z \simeq e^{-S_{E}}$, where the Euclidean action $S_{E}$ is evaluated on an on-shell field configuration satisfying suitable boundary conditions [28].

Employing this, we obtain a relation between the Euclidean on-shell action and the free energy:

$$
\log (Z) \simeq-S_{E} \simeq-\beta F \quad \Rightarrow \quad F \simeq \frac{S_{E}}{\beta}
$$

When gauge fields are present, the boundary conditions are chosen such that the total charge $\mathcal{Q}$ is fixed. Then the Euclidean action depends on the associated chemical potential $\mu$, so that $S_{E}=S_{E}(\beta, \mu)$, and one obtains the following relation between the Euclidean on-shell action and the grand potential $\Omega(\beta, \mu)$ :

$$
\log (\mathcal{Z}) \simeq-S_{E} \simeq-\beta \Omega, \quad \Rightarrow \quad \Omega \simeq \frac{S_{E}}{\beta}
$$

\subsection{Simple Wick-rotation}

We now use the Einstein-Maxwell theory with a cosmological constant to review the standard Wick-rotation. Our conventions for actions are explained in appendix A. We follow [29] for the gravitational action, and generalise this by including the cosmological constant and the Maxwell action:

$$
\begin{aligned}
S & =S_{\text {bulk }}+S_{\mathrm{GHY}} \\
= & -\frac{1}{16 \pi} \int_{M} \sqrt{|g|}(R-2 \Lambda) d^{4} x-\frac{1}{16 \pi} \int_{M} \sqrt{|g|} F_{\mu \nu} F^{\mu \nu} d^{4} x \\
& +\frac{\epsilon}{8 \pi} \int_{\partial M} \sqrt{|\gamma|}\left(K-K_{0}\right) d^{3} x .
\end{aligned}
$$

The middle line is the bulk term, containing the Einstein-Hilbert action with the Ricci scalar $R$, a cosmological constant $\Lambda$ and the Maxwell term. The second line is the GibbonsHawking-York boundary term $S_{\mathrm{GHY}}[4,30]$, which is needed to cancel boundary terms arising from the variation of the Einstein-Hilbert action if spacetime is not closed (compact without boundary). The spacetime metric $g$ induces a metric $\gamma$ on the boundary $\partial M . K$ is trace of the extrinsic curvature of $\partial M$ as an embedded submanifold of spacetime $M$, see the appendix B for details. The constant $\epsilon$ takes the values $\epsilon= \pm 1$ for boundaries with unit normals which are either spacelike $(+)$ or timelike $(-)$. To obtain a finite value for the on-shell action, we include a background term $K_{0}$. For an asymptotically flat spacetime $K_{0}$ is the extrinsic curvature of the boundary embedded into a flat spacetime, which ensures that the action of Minkowski space, which is a solution for $\Lambda=0$, is zero rather than divergent. 
We now apply the Wick-rotation $t \rightarrow-i t$ to $(2.1)$ to map $\exp (i S) \rightarrow \exp \left(-S_{E}\right)$. Following [29] we first consider the gravitational terms. The bulk gravitational term receives a factor of $-i$ from the measure:

$$
-\frac{1}{16 \pi} \int_{M} \sqrt{g}(R-2 \Lambda) d^{4} x \rightarrow i \frac{1}{16 \pi} \int_{M} \sqrt{g}(R-2 \Lambda) d^{4} x .
$$

For the transformation of the GHY-term we need to distinguish two cases.

1. For surfaces with a timelike unit normal:

$$
\epsilon=-1, \quad K \rightarrow i K, \quad \sqrt{\gamma} d^{3} x \rightarrow \sqrt{\gamma} d^{3} x .
$$

2. For surfaces with a spacelike unit normal:

$$
\epsilon=1, \quad K \rightarrow K, \quad \sqrt{\gamma} d^{3} x \rightarrow-i \sqrt{\gamma} d^{3} x .
$$

The resulting Euclidean Gibbons-Hawking-York term is the same for both types of hypersurfaces and transforms as

$$
+\frac{\epsilon}{8 \pi} \int_{\partial M} \sqrt{|\gamma|}\left(K-K_{0}\right) d^{3} x \rightarrow-i \frac{1}{8 \pi} \int_{\partial M} \sqrt{|\gamma|}\left(K-K_{0}\right) d^{3} x .
$$

We now consider the Maxwell field. Before Wick-rotation, we use that the Maxwell action is evaluated on-shell, allowing us to rewrite its contribution as a total derivative ${ }^{5}$

$$
F^{\mu \nu} F_{\mu \nu}=2 \nabla_{\mu}\left(A_{\nu} F^{\mu \nu}\right) .
$$

Applying Stoke's theorem, we can write the bulk contribution as an integral over the boundary

$$
-\frac{1}{8 \pi} \int_{M} \sqrt{|g|} \nabla_{\mu}\left(A_{\nu} F^{\mu \nu}\right) d^{4} x=\frac{1}{8 \pi} \int_{\partial M} F^{\mu \nu} A_{\mu} d \Sigma_{\nu},
$$

where the volume element on the boundary is defined as $d \Sigma_{\mu}=n_{\mu} \sqrt{|\gamma|} d^{3} x$ and $n^{\mu}$ is the outward-pointing unit normal vector. Applying a Wick-rotation, we find the Maxwell action transforms as

$$
\frac{1}{8 \pi} \int_{\partial M} F^{\mu \nu} A_{\mu} d \Sigma_{\nu} \rightarrow-i \frac{1}{8 \pi} \int_{\partial M} F^{\mu \nu} A_{\mu} d \Sigma_{\nu}
$$

where note explicitly that each pieces transforms as: $d \Sigma_{\mu} \rightarrow-i d \Sigma_{\mu}, A_{\mu} \rightarrow-i A_{\mu}$, and $F^{\mu \nu} \rightarrow i F^{\mu \nu}$.

Taking all contributions together, the Euclidean action is

$$
\begin{aligned}
S_{E}=-i S_{\text {Wick-rotated }}= & \frac{1}{16 \pi} \int_{M} \sqrt{g}(R-2 \Lambda) d^{4} x \\
& -\frac{1}{8 \pi} \int_{\partial M} \sqrt{|\gamma|}\left(K-K_{0}\right) d^{3} x-\frac{1}{8 \pi} \int_{\partial M} F^{\mu \nu} A_{\mu} d \Sigma_{\nu} .
\end{aligned}
$$

\footnotetext{
${ }^{5}$ In terms of differential forms, $F \wedge \star F=d A \wedge \star F=d(A \wedge \star F)$, if $d \star F=0$.
} 


\subsection{Triple Wick-rotation}

The standard simple Wick-rotation can be applied for static spacetimes which upon continuation remain real, so that the Euclidean on-shell action can be interpreted as a thermal partition function. The static patches of the planar solutions found in [24] take the form

$$
d s^{2}=-f(r) d t^{2}+\frac{d r^{2}}{f(r)}+r^{2}\left(d x^{2}+d y^{2}\right),
$$

which at first appears suitable for this procedure. However, we also need smooth field configurations to obtain a well-defined and finite Euclidean on-shell action. For the solutions of [24] the static patches have a curvature singularity for some finite value $r=r_{\text {sing }}$ of the transverse coordinate $r$, which makes the Euclidean on-shell action ill-defined.

However, these static patches have a horizon at another finite value $r=r_{h}>r_{\text {sing }}$ of the coordinate $r$, and by analytic continuation one obtains a dynamic patch, where the metric, after relabelling $r \leftrightarrow t$, takes the form

$$
d s^{2}=-\frac{d t^{2}}{\tilde{f}(t)}+\tilde{f}(t) d r^{2}+t^{2}\left(d x^{2}+d y^{2}\right) .
$$

Note that the function $\tilde{f}(t)$ has been modified with an additional sign: $\tilde{f}(x)=-f(x)$. This ensures that $\tilde{f}(t)$ is positive definite within the domain of $t \in\left(t_{h}, \infty\right)$. For the remainder of the discussion, the tilde will be dropped and it is understood that functions $f$ appearing in the line element are positive definite for each patch, and that the coordinate denoted $t$ is timelike while the coordinate denoted $r$ is spacelike.

It was shown in [24] that these solutions have a well behaved asymptotic behaviour for $t \rightarrow \infty .^{6}$ In the dynamic patch, the horizontal Killing vector field is spacelike rather than timelike, and the application of the simple Wick-rotation leads to a complex line element and action. To work with this dynamic patch, we will need to modify the standard Euclidean method. There are some examples where complex line elements are used in the literature, the canonical example being the Kerr metric [4]. In this case, the generalisation is to admit timelike Killing vector fields which are not hypersurface orthogonal, and the complexification arises from cross terms in the line element. This is different from our case, where the Killing vector field is still hypersurface orthogonal, but spacelike.

We therefore explore an alternative procedure, which in principle can be applied to any metric which has no timelike-spacelike cross-terms, and depends explicitly on time but not on the spatial coordinates. We choose to Wick-rotate all three spacelike coordinates of the line element. Since the examples for which we will obtain a well defined Euclidean action are of the form (2.4), we denote the spatial coordinates $r, x, y$ so that the triple Wick-rotation takes the form

$$
(r, x, y) \rightarrow \pm i(r, x, y)
$$

where we admit either choice of sign. As we work with the mostly plus conventions, the resulting Euclidean line element will be negative-definite.

\footnotetext{
${ }^{6}$ To be precise, there are two extensions of the static patch, and depending on the extension, $t \rightarrow \infty$ either corresponds to future or to past timelike infinity. We refer to the appendix D for a discussion of the global structure of the solution.
} 
Applying this transformation to (2.1) the Euclidean action associated with the triple Wick-rotation is calculated. The bulk contribution transforms as

$$
\begin{aligned}
-\frac{1}{16 \pi} \int_{M}(R-2 \Lambda) \sqrt{-g} d^{4} x & \rightarrow-( \pm i)^{3} \frac{1}{16 \pi} \int_{M}(R-2 \Lambda) \sqrt{-g} d^{4} x \\
& = \pm i \frac{1}{16 \pi} \int_{M}(R-2 \Lambda) \sqrt{-g} d^{4} x
\end{aligned}
$$

The GHY-term, as with the single Wick-rotation, transforms with the same sign for $\epsilon= \pm 1$.

1. For surfaces with a timelike unit normal

$$
\epsilon=-1, \quad K \rightarrow K, \quad \sqrt{\gamma} d^{3} x \rightarrow( \pm i)^{3} \sqrt{\gamma} d^{3} x
$$

2. For surfaces with a spacelike unit normal,

$$
\epsilon=1, \quad K \rightarrow \mp i K, \quad \sqrt{\gamma} d^{3} x \rightarrow( \pm i)^{2} \sqrt{\gamma} d^{3} x
$$

and we see that for either hypersurface, the GHY term transforms under a triple Wickrotation as

$$
+\frac{\epsilon}{8 \pi} \int_{\partial M} \sqrt{|\gamma|}\left(K-K_{0}\right) d^{3} x \rightarrow \pm i \frac{1}{8 \pi} \int_{\partial M} \sqrt{|\gamma|}\left(K-K_{0}\right) d^{3} x .
$$

As with the standard Wick-rotation, we can write the gauge field contribution as a boundary term as we evaluate the action on-shell. Performing the triple Wick-rotation, we find

$$
\frac{1}{8 \pi} \int_{\partial M} F^{\mu \nu} A_{\mu} d \Sigma_{\nu} \rightarrow \mp i \frac{1}{8 \pi} \int_{\partial M} F^{\mu \nu} A_{\mu} d \Sigma_{\nu}
$$

where we have used that $d \Sigma_{\mu} \rightarrow( \pm i)^{3} d \Sigma_{\mu}, A_{\mu} \rightarrow \pm i A_{\mu}$ and $F^{\mu \nu} \rightarrow \mp i F^{\mu \nu}$. Piecing this all together, the triple Wick-rotated Euclidean action is given by

$$
\begin{aligned}
S_{E}= & \pm \frac{1}{16 \pi} \int_{M} \sqrt{g}(R-2 \Lambda) d^{4} x \\
& \pm \frac{1}{8 \pi} \int_{\partial M} \sqrt{|\gamma|}\left(K-K_{0}\right) d^{3} x \mp \frac{1}{8 \pi} \int_{\partial M} F^{\mu \nu} A_{\mu} d \Sigma_{\nu}
\end{aligned}
$$

We then identify the thermodynamic potential as we do in the standard formulation, evaluating the partition function $\mathcal{Z}$ in a saddle point approximation to obtain

$$
\log \mathcal{Z}=-S_{E}(\beta, \mu)=-\beta \Omega,
$$

where the inverse temperature $\beta$ and chemical potential $\mu$ can be expressed in terms of parameters of the triple-Wick rotated solution. 


\subsection{Surface gravity and temperature}

When working with the Euclidean action formalism, the temperature associated with a Killing horizon is usually determined by the periodicity of Euclidean time, which in turn is fixed by imposing the absence of a conical singularity after Wick-rotation. The near horizon approximation of the line element has the Rindler-like metric

$$
d s^{2}=-\kappa^{2} r^{2} d t^{2}+d r^{2}+(A+\cdots) d \vec{X}^{2},
$$

where $\kappa$ is the surface gravity, $r^{\prime}=r-r_{h}$ is a shifted coordinate which vanishes at the horizon, and $A$ is independent on $r^{\prime}$. The term $d \vec{X}^{2}$ is the standard line element on the unit-sphere or on the Euclidean plane, depending on whether we impose spherical or planar symmetry. While the term proportional to $d \vec{X}^{2}$ in the line element is manifestly regular for $r^{\prime}=0$, there is a conical singularity in $\left(t, r^{\prime}\right)$-plane unless the Euclidean time coordinate $t$ satisfies $\kappa t \sim \kappa t+2 \pi$ and thus is periodic with period $2 \pi \kappa^{-1}$. This determines the temperature $T_{H}$ associated with the horizon, $\beta=T_{H}^{-1}=2 \pi \kappa^{-1}$. We observe that since the surface gravity $\kappa$ enters the line element quadratically, this procedure does not actually determine whether $T_{H}$ is positive or negative. However, the sign can be set through computing the Hawking temperature using curved spacetime QFT [3], or the tunneling effect for a quantum particle [31]. As an aside, we note that by removing the conical singularity, the horizon becomes the origin in the Wick-rotated spacetime. As a result, the Wick-rotated spacetime has only one boundary, located at $r \rightarrow \infty$. This means that when we calculate boundary terms for the Euclidean action, there will only be asymptotic contributions.

The surface gravity $\kappa$ of a Killing horizon is defined by

$$
\left.\xi^{\nu} \nabla_{\nu} \xi^{\mu}\right|_{r=r_{h}}=\kappa \xi^{\mu}
$$

evaluated on the horizon, where $\xi$ is a Killing vector field which is null on the horizon and non-null outside the horizon. We observe that $\kappa$ changes sign under $\xi \rightarrow-\xi$. For static, asymptotically flat spacetimes, the sign can be fixed by defining $\kappa$ to be the acceleration of a test mass at the horizon, multiplied by the redshift factor [32]. This also fixes the magnitude of $\kappa$, which changes under rescalings of $\xi$. The standard formula (2.7) applies to the case where $\xi$ has unit norm at spatial infinity.

Since we will investigate a non-standard situation, we will not assume that $\kappa$ and $T_{H}$ are positive. Furthermore, as the asymptotic region is not flat, we have to specify how we normalize the horizontal Killing vector field. For this purpose we will follow the work of [33] and $[34,35]$, which provides a method for computing the surface gravity and temperature of trapping horizons. While the definition of an event horizon requires the knowledge of the global causal structure of a spacetime, trapping horizons are defined quasi-locally by the existence of marginally trapped surfaces. That is, on a trapping horizon the expansion of one of the two future-directed null congruences defined by ingoing and outgoing light rays changes sign, so that the horizon separates a non-trapping region where one congruence expands and the other contracts from a trapping region where both congruences either expand or contract. The Killing horizons of the solutions [24] are event horizons, and thus 
in particular trapping horizons, so that the formalism can be applied. ${ }^{7}$ In the literature the term 'trapping horizon' is used for hypersurfaces where the expansion of one null congruence vanishes, while spatial cross sections of a trapping horizon are called 'apparent horizons.' We will use both terms interchangeably.

In the so-called Kodama-Hayward approach $[33,36]$, the surface gravity is obtained as follows. The metric is required to have the structure

$$
d s^{2}=\gamma_{i j}(x) d x^{i} d x^{j}+C^{2}(x) d \vec{X}^{2}, \quad i=0,1,
$$

where $\gamma_{i j}{ }^{8}$ and $C$ only depend on the coordinates $\left(x^{0}, x^{1}\right)=(t, r)$. For spherically symmetric spacetimes, $d \vec{X}^{2}=d \Omega_{2}$ is the standard metric on the two-sphere. In our calculations, we allow planar symmetry and hence $d \vec{X}^{2}$ is the standard metric on $\mathbb{R}^{2}$. The surface gravity in the Kodama-Hayward formalism is

$$
\kappa=\frac{1}{2 \sqrt{-\gamma}} \partial_{i}\left(\sqrt{-\gamma} \gamma^{i j} \partial_{i} C\right)=\frac{1}{2} \Delta_{\gamma} C .
$$

For later reference, we compute the Kodama-Hayward surface gravity for line elements of the form

$$
d s^{2}=-\frac{d t^{2}}{f(t)}+f(t) d r^{2}+t^{2} d \vec{X}^{2}
$$

which include the dynamic patches of [24], and obtain

$$
\kappa=-\frac{1}{2} \partial_{t} f(t)
$$

Following [34, 35], trapping horizons and their Kodama-Hayward surface gravity subdivide into four cases, as follows. One chooses a local frame containing two future-directed null vectors, $N_{ \pm}^{\mu}$ which are 'outgoing' $(+)$ and 'ingoing' $(-)$. Using the definition for the expansion

$$
\theta_{ \pm}=\nabla_{\mu} N_{ \pm}^{\mu}
$$

the four types of horizons are determined by calculating their expansions $\theta_{ \pm}$and their Lie derivative, evaluated on the horizon.

Non-trapping regions in spacetime are those where $\theta_{+} \theta_{-}<0$. The convention taken in $[34,35]$ is that $\theta_{-}<0$ and $\theta_{+}>0$ in all non-trapping regions so that the outgoing congruence $N_{+}^{\mu}$ is diverging (or expanding) while the ingoing congruence $N_{-}^{\mu}$ is converging (or contracting). Trapping regions are those where $\theta_{+} \theta_{-}>0$, so that either both congruences expand, or both congruences contract. Apparent horizons occur at boundaries where $\theta_{ \pm}=0, \theta_{\mp} \neq 0$.

In appendix $\mathrm{D}$, we construct the maximal extensions of spacetimes with a line element of the form (2.3), using only qualitative properties of the function $f$, namely its zeros and

\footnotetext{
${ }^{7}$ While [33] and [35] assume spherical symmetry, their formalism extends straightforwardly to situations with planar symmetry.

${ }^{8}$ Note that here $\gamma$ is the metric for the transverse coordinates and is distinct from the boundary metric $\gamma$ used in our Euclidean action calculations. As it is unlikely to cause confusion, we allow this duplicity in our notation in order that this section uses the same conventions as the cited papers [34, 35].
} 
asymptotic behaviour. We compute the expansions of null congruences, identify the types of the trapping horizons and identify the global causal structure. For comparison, we also include spacetimes like the Schwarzschild spacetime where the static region is $r>r_{h}$ rather than $r<r_{h}$. In all cases the maximally extended spacetime consists of four regions, which are separated by trapping horizons which happen to be Killing horizons. We identify one of the two non-trapping regions with the region where the line element (2.3) is static, and use this 'standard static patch' (or standard non-trapping patch) to fix the direction of physical time for the extended spacetime. In this patch we identify two future-pointing null geodesic congruences $N_{ \pm}^{\mu}$, such that their expansions satisfy $\theta_{+}>0$ and $\theta_{-}<0$. Then we choose Kruskal-like coordinates $\left\{X_{+}, X_{-}, \ldots\right\}$ in such a way that they are adapted to the standard patch, that is, such that in the standard patch ingoing future-pointing null congruences have constant $X_{+}$and propagate towards increasing $X_{-}$, while outgoing future-pointing null congruences have constant $X_{-}$and propagate towards increasing $X_{+}$. When extending the metric, the vector fields $N_{ \pm}^{\mu}$ and the scalars $\theta_{ \pm}$to the full maximally extended spacetime, we observe that there always is a second static, non-trapping patch. In this second static patch the roles of ingoing and outgoing congruences are reversed so that we have the non-standard assignments $\theta_{+}<0$ and $\theta_{-}>0$. In addition, there always are two trapping regions, one where both congruences expand, one where both contract. The transverse coordinate $r$ used in (2.3) can always be extended beyond the trapping horizon and covers two regions of the extended spacetime, depending on the choice of the static patch and the way in which we continue. We call regions interior regions when they contain a curvature singularity, and exterior regions if null geodesics can be extended to infinite affine parameter in one direction. The coordinate $r$ takes values $r<r_{h}$ in the interior and $r>r_{h}$ in the exterior regions. For our main examples, the de Sitter, planar Einstein-Maxwell and planar STU solutions, the inner region $r<r_{h}$ is static while the exterior region $r>r_{h}$ is dynamic. We will therefore relabel $r \rightarrow t$ in exterior regions to emphasize that this cooordinate is timelike. For more details, we refer the reader to appendix D.

The variation of the expansions are given by their Lie derivatives $\mathcal{L}_{ \pm} \theta_{\mp}$ with respect to the lightcone coordinates $X_{ \pm}$. Since $\mathcal{L}_{ \pm} \theta_{\mp}$ does not change sign across horizons, there are four types of horizons $[34,35]$.

1. Future outer horizons: $\theta_{+}=0, \theta_{-}<0, \mathcal{L}_{N_{-}} \theta_{+}<0$. The sign of $\theta_{+}$changes from positive to negative with growing $X_{-}$. The situation is analogous to regions I and II of the extended Schwarzschild solution, see the left diagram in figure 6. For sufficiently small $X_{-}$('outside the horizon') the outgoing congruence is expanding, while for sufficiently large $X_{-}$('inside the horizon') both congruences contract. Therefore future outer horizons can be taken as local definitions of black holes.

2. Past outer horizons: $\theta_{-}=0, \theta_{+}>0, \mathcal{L}_{N_{+}} \theta_{-}<0$. The sign of $\theta_{-}$changes from positive to negative with growing $X_{+}$. For sufficiently large $X_{+}$('outside the horizon') the ingoing congruences are converging, while for sufficiently small $X_{+}$('inside the horizon') both congruences expand. The small $X_{+}$region is analogous to the timereflected region IV of the extended Schwarzschild solution, while the large $X_{+}$region 
is analogous to region I. Therefore past outer horizons can be taken as local definitions of white holes.

3. Future inner horizons: $\theta_{+}=0, \theta_{-}<0, \mathcal{L}_{N_{-}} \theta_{+}>0$. The sign of $\theta_{+}$changes from negative to positive with increasing $X_{-}$. The inside region (large $X_{-}$) is non-trapping while in the outside region (small $X_{-}$) both congruences contract. Therefore future inner horizons can be taken as local definitions of contracting cosmologies, where all null congruences become converging for large enough distances from the observer.

4. Past inner horizons: $\theta_{-}=0, \theta_{+}>0, \mathcal{L}_{N_{+}} \theta_{-}>0$. The sign of $\theta_{-}$changes from negative on the inside (small $X_{+}$) to positive on the outside (large $X_{+}$). The interior region is non-trapping while in the exterior region both congruences expand. Therefore past inner horizons are local definitions of expanding cosmologies, where all null congruences become expanding for large enough distances from the observer.

The surface gravities of these horizons are related to variations of the expansions by $\kappa \propto$ $-\mathcal{L}_{ \pm} \theta_{\mp}$. Thus outer horizons have positive surface gravity, while inner horizons have negative surface gravity. A further sign has been argued for in the relation between surface gravity and temperature. In $[34,35]$ the Hawking temperature of an apparent horizon was computed using the Parikh-Wilczek tunnelling method. It was found that $T_{H} \propto \pm \kappa$, with the upper sign for future horizons and the lower sign for past horizons. The net effect is that future outer horizons (black holes), and past inner horizons (expanding cosmologies) have positive temperature, while future inner horizons (contracting cosmologies) and past outer horizons (white holes) have negative temperature.

Negative temperature was argued to indicate the absence of Hawking radiation, since future inner and past outer horizons cannot separate virtual particle pairs created by vacuum fluctuations, thus not enabling the Hawking effect [34, 35]. In thermodynamics, the inverse temperature is related to the entropy $S$ and internal energy $E$ by

$$
\beta=\frac{\partial S}{\partial E}
$$

Therefore, negative temperature can occur if one drops the usual assumption that the entropy increases monotonically with the energy. A toy model for negative temperature is provided by a system with finite maximum energy [37]. Taking a system with two energy eigenstates $E_{1}<E_{2}$ as the simplest example, this will be in a maximally ordered state, $S=0$, if all particles are either in the lower or in the higher state, while a maximally disordered state is realized when half of the particles are in either state. Upon heating up such a system, entropy and temperature first increase, with the temperature reaching $+\infty$ when entropy becomes maximal. Upon further heating, the entropy decreases and the temperature jumps at the turning point from $+\infty$ to $-\infty$. After this point, it increases, approaching 0 from below when reaching a situation where all particles are in the higher state. Thus negative temperatures are 'higher' than positive temperatures and correspond to 'population inversion.' We will see later that some of the horizons we are interested in have negative surface gravity and negative temperature, and that this is necessary in order for the first law to take its standard form when using our triple Wick rotated Euclidean formalism. 


\section{Thermodynamics of the de Sitter solution}

As an introductory example of the implementation of the triple Wick-rotation in spacetimes with dynamic asymptotic regions, we study the de Sitter solution of Einstein's equations with a cosmological constant. This example is somewhat simpler than the solutions of [24] since it is a vacuum solution. However, it allows us to demonstrate that the results we obtain using a triple Wick-rotation in the dynamic patch agree with those obtained previously using a single Wick-rotation in the static patch.

\subsection{Static patch, single Wick-rotation}

The de Sitter spacetime line element in static coordinates is given by

$$
d s^{2}=-\left(1-\frac{r^{2}}{L^{2}}\right) d t^{2}+\left(1-\frac{r^{2}}{L^{2}}\right)^{-1} d r^{2}+r^{2} d \Omega_{2}^{2},
$$

with the cosmological horizon located at $r_{h}=L$, where $L$ is the de Sitter radius and the domain of our radial coordinate is $r \in\left[0, r_{h}\right)$. At $r=r_{h}$ there is a Killing horizon for the Killing vector field $\xi=\partial_{t}$, which becomes spacelike when we continue to $r>r_{h}$. The thermodynamics of de Sitter space can be calculated within the static patch $0<r<$ $r_{h}$ using standard methods. The cosmological constant $\Lambda$ can be written generally as a function of the de Sitter radius

$$
\Lambda=-\frac{(d-1)(d-2)}{2 L^{2}}=-\frac{3}{L^{2}},
$$

where for reference, we first give the relation for general dimension $d$ before setting $d=4$. Note the minus sign, which is due to our sign conventions where the cosmological constant is proportional to the Ricci scalar, while the Ricci scalar is negative for de Sitter space. We expand on our conventions in appendix A.

Under the Wick-rotation $t \rightarrow-i \tau$, the line element (3.1) maps to the positive definite line element

$$
d s^{2}=\left(1-\frac{r^{2}}{L^{2}}\right) d \tau^{2}+\left(1-\frac{r^{2}}{L^{2}}\right)^{-1} d r^{2}+r^{2} d \Omega_{2}^{2} .
$$

Entropy. Using the Bekenstein-Hawking area law, the entropy is determined by

$$
S_{d S}=\frac{A}{4}=\pi^{2} L .
$$

Temperature. The temperature associated with the horizon is proportional to the Kodama-Hayward surface gravity, which is found to be $\kappa=-L^{-1}$, thus yielding the Hawking temperature

$$
T_{H}=\frac{\kappa}{2 \pi}=-\frac{1}{2 \pi L} .
$$

Note that the Hawking temperature is negative. We employ the definitions of $[34,35]$ and consider horizons which can be crossed by future directed null rays and future directed time-like curves ('observers') from the outside to the inside. These are the regions III and IV in figure 1, where the global time orientation is chosen such that the Killing vector 


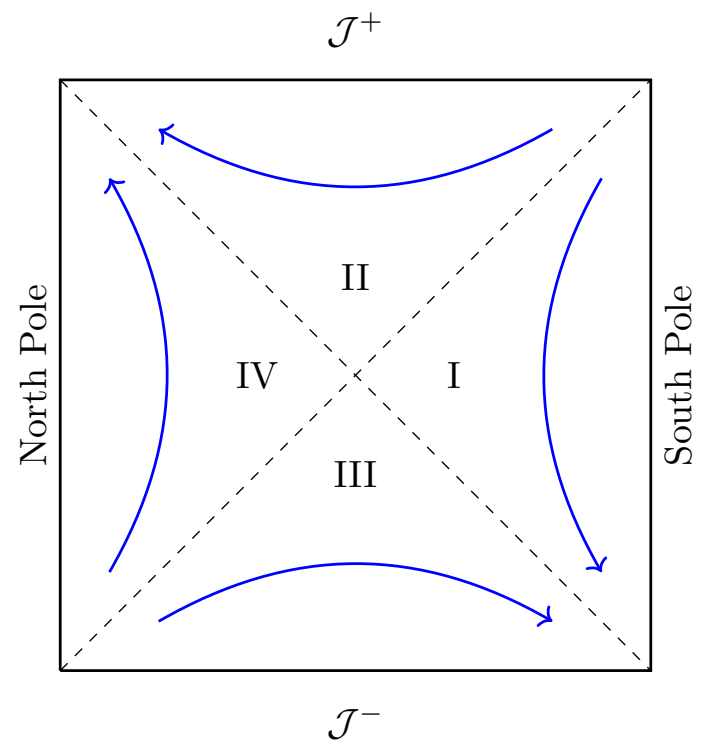

Figure 1. Penrose-Carter diagram for the global de Sitter solution. Dashed lines denote the cosmological horizon located for $r=L$ and the North/South poles are identified for $r=0$. Blue curved arrows denote the flow of the Killing vector field.

field is future-pointing in region III, that is, globally time flows 'upwards'. This choice of regions is natural because it has the same causal structure as the part of the extended Schwarzschild spacetime which describes a black hole (regions I and II in the left diagram of figure 6). As we show in appendix D, the horizon between regions III and IV in the global de Sitter spacetime is a future inner horizon, which therefore has negative surface gravity and temperature. This is different from the assignments made in other references, including [5, 38], where the Hawking temperature is positive: $T_{H}>0$. However according to [38] this implies that the entropy is negative. A positive temperature for de Sitter horizons is consistent with considering the past inner horizon separating regions IV and II. In contrast, in this paper the sign of the temperature is determined by the type of apparent horizon, but the entropy is always defined by the area law and therefore positive. Note that the expression $T d S$ entering into the first law is the same in both approaches.

Euclidean action. Global de Sitter space is a maximally symmetric space of constant positive curvature with topology $\mathbb{R} \times S^{3}$. Its Kruskal diagram decomposes into four regions, two of which have a timelike Killing vector field and do not intersect the boundary, which is spacelike with topology $S^{3}$. If we evaluate the Euclidean action on a static patch, the boundary terms do not contribute and the de Sitter action is completely determined by the bulk terms:

$$
S_{E}=\frac{1}{16 \pi} \int_{M} \sqrt{g}(R-2 \Lambda),
$$

where the Ricci curvature is constant: $R=-12 L^{-2}$ and the integral over the four-manifold gives

$$
S_{E}=\frac{1}{16 \pi}\left(-\frac{6}{L^{2}}\right) \int_{0}^{\beta} d \tau \int_{S^{2}} \sin \theta d \theta d \phi \int_{r_{h}}^{0} r^{2} d r=-\pi L^{2}
$$


Note the limits on the integration of the radial coordinate $r$, which have been chosen to run from $r_{h}$, the origin of the Euclidean manifold, to the North pole for $r=0 .{ }^{9}$ As there are no charges in the solution, we work in the canonical ensemble and we have the following relations:

$$
\log (Z)=-S_{E}=-\beta F, \quad F=E-T S .
$$

The de Sitter solution is a maximally symmetric vacuum solution and thus interpreted as a ground state. We therefore choose the natural normalisation $E=0$. Following from this we obtain

$$
S_{E}=\beta F=-S \quad \Rightarrow \quad S=\pi L^{2} .
$$

We see that the thermodynamic entropy matches with (3.3) and the first law is satisfied though in a 'degenerate way', as the entropy is constant: $d S=0=T d S=d E=d(0)=0$.

\subsection{Dynamic patch, triple Wick-rotation}

The static patch is not complete and by analytical extension of the coordinate $r$ through the Killing horizon to values $r>r_{h}$ we obtain a second, dynamical patch, with asymptotic region $r \rightarrow \infty$. When crossing the horizon, the function $f(r)$ becomes strictly negative, and we find that the coordinates $t, r$ exchange their roles. The timelike coordinate $t$ becomes spacelike, while the spacelike coordinate $r$ becomes timelike. We adopt the convention to relabel coordinates in the dynamic patch, so that $t$ is always timelike and $r$ always spacelike.

Then the line element in the dynamic patch is

$$
d s^{2}=-\left(\frac{t^{2}}{L^{2}}-1\right)^{-1} d t^{2}+\left(\frac{t^{2}}{L^{2}}-1\right) d r^{2}+t^{2} d \Omega_{2}^{2}
$$

The coordinate domain is $t \in\left(t_{h}, \infty\right)$ where $t_{h}$ is the Killing horizon located at $t_{h}=L$. Note that while this cannot be read of from the local form of the line element, we have chosen the continuation from region IV to region III, so that $t \rightarrow \infty$ corresponds to past timelike infinity, see appendix $\mathrm{D}$ for details. This is relevant because it determines the sign of the temperature.

Triple Wick-rotation. We now perform a triple Wick-rotation where $r \rightarrow \pm i r$ and where the sphere $S^{2}$ is analytically continued to the hyperbolic plane $\mathcal{H}_{2}$ by $(\theta, \phi) \rightarrow$ $\pm i(\theta, \phi)$. The line element (3.4) is mapped to the negative-definite line element

$$
d s^{2}=-\left(\frac{t^{2}}{L^{2}}-1\right)^{-1} d t^{2}-\left(\frac{t^{2}}{L^{2}}-1\right) d r^{2}-t^{2} d \mathcal{H}_{2}^{2}, \quad d \mathcal{H}_{2}^{2}=d \theta^{2}+\sinh ^{2} \theta d \phi^{2} .
$$

\footnotetext{
${ }^{9}$ As this might be confusing, let us justify the integration bounds. Although we interpret $r=0$ as the coordinate origin for static coordinates of the de Sitter solution, this is not the origin for the Wick-rotated Euclidean manifold. When we Wick-rotate, the location of the horizon: $r=r_{h}$, becomes the origin with the identification $\tau \simeq \tau+\beta$ made to avoid a conical singularity. Our integration limits are then chosen to match the conventions from the origin of the Euclidean space to the boundary and as such we integrate from $r=r_{h}$ to $r=0$.
} 
Temperature \& entropy. The temperature associated to the Killing horizon is the same as in the previous calculation. Using the Kodama-Hayward expression (2.8), the surface gravity is found to be $\kappa=-L^{-1}$ and for a future inner horizon the Hawking temperature is

$$
T_{H}=\frac{\kappa}{2 \pi}=-\frac{1}{2 \pi L} .
$$

The entropy is identical to the static solution and is given by

$$
S_{d S}=\frac{A}{4}=\pi^{2} L
$$

Euclidean action. The dynamical patches of global de Sitter space intersect the boundary, which is spacelike with topology $S^{3}$. Therefore we need to take boundary terms into account. After our triple Wick-rotation, the boundary has topology $S^{1} \times \mathcal{H}_{2}$, where the radius of the $S^{1}$ is fixed by imposing the absence of a conical singularity.

The Euclidean action for the triple-Wick-rotated system is

$$
S_{E}= \pm \frac{1}{16 \pi} \int_{M} \sqrt{g}(R-2 \Lambda) d^{4} x \pm \frac{1}{8 \pi} \int_{\partial M} \sqrt{\gamma} K d^{3} x+\int_{\partial M} L_{c t}[\gamma] d^{3} x,
$$

where a counter term $L_{c t}$ has been included to remove divergences from the action. The boundary is at $t \rightarrow \infty$, and we first integrate $t$ in the domain $t \in\left[t_{h}, \epsilon^{-1}\right)$ and then take the limit of $\epsilon \rightarrow 0$. The volume

$$
\omega=\int_{\mathcal{H}_{2}} \sinh \theta d \theta d \phi
$$

of the hyperbolic plane is divergent. While one option in this situation is to work with densities, we keep $\omega$ as a formal constant which corresponds to the parametric volume $\omega_{S^{2}}=4 \pi$ of the two-sphere in the static patch.

The bulk term of the Euclidean action is

$$
S_{\mathrm{Bulk}}= \pm \frac{1}{16 \pi} \int_{M} \sqrt{g}(R-2 \Lambda) d^{4} x
$$

where

$$
R=-\frac{12}{L^{2}}, \quad \Lambda=-\frac{3}{L^{2}}, \quad \sqrt{g}=t^{2} \sinh \theta .
$$

Putting these into the action and integrating over the manifold we find:

$$
\begin{aligned}
S_{\mathrm{Bulk}} & = \pm \frac{1}{16 \pi} \int_{M} \sqrt{g}(R-2 \Lambda) d^{4} x \\
& = \pm \frac{1}{16 \pi}\left(-\frac{6}{L^{2}}\right) \int_{0}^{\beta} d r \int_{\mathcal{H}_{2}} \sinh \theta d \theta d \phi \int_{L}^{\epsilon^{-1}} d t t^{2} \\
& =\mp \frac{\beta \omega}{16 \pi} \frac{2}{L^{2}}\left(\frac{1}{\epsilon^{3}}-L^{3}\right) .
\end{aligned}
$$

The Gibbons-Hawking-York term

$$
S_{\mathrm{GHY}}= \pm \frac{1}{8 \pi} \int_{\partial M} \sqrt{-\gamma} K d^{3} x
$$


can be calculated in the following way: the normal vector to the boundary for constant $t$ is

$$
n^{\mu}=(-\sqrt{f}, 0,0,0) \quad \Rightarrow \quad n_{\mu} n^{\mu}=-1 .
$$

The trace $K$ of the extrinsic curvature, evaluated on a surface of constant $t=t_{0}$, can then be computed using (B.7):

$$
\begin{gathered}
K=\nabla_{\mu} n^{\mu}=\frac{3 t_{0}^{2}-2 L^{2}}{t_{0} L^{2} \sqrt{f}}, \quad \sqrt{-\gamma}=\sqrt{f\left(t_{0}\right)} t_{0}^{2} \sinh \theta, \\
K \sqrt{-\gamma}=\frac{3 t_{0}^{3}}{L^{2}}-2 t_{0} .
\end{gathered}
$$

Combining these, we find that the boundary contribution at $t_{0}=\epsilon^{-1}$ is:

$$
\begin{aligned}
S_{\mathrm{GHY}} & = \pm \frac{1}{8 \pi} \int_{\partial M} \sqrt{-\gamma} K d^{3} x \\
& = \pm \frac{1}{8 \pi}\left(\frac{3}{L^{2} \epsilon^{3}}-\frac{2}{\epsilon}\right) \int_{0}^{\beta} d r \int_{\mathcal{H}_{2}} \sinh \theta d \theta d \phi \\
& = \pm \frac{\beta \omega}{8 \pi}\left(-\frac{2}{\epsilon}+\frac{3}{L^{2} \epsilon^{3}}\right) .
\end{aligned}
$$

The counter term is constructed from the geometric data of the boundary metric:

$$
\int_{\partial M} L_{c t}[\gamma] d^{3} x=\int_{\partial M} d^{3} x \sqrt{|\gamma|}\left(c_{1}+c_{2} R[\gamma]\right),
$$

where $R[\gamma]$ is the Ricci curvature associated to the boundary manifold, and $c_{1,2}$ are renormalisation constants. We can expand out the counter terms in orders of $\epsilon$ and find:

$$
\begin{aligned}
\sqrt{|\gamma|} & =\left(\frac{1}{L \epsilon^{3}}-\frac{L}{2 \epsilon}+\mathcal{O}\left(\epsilon^{1}\right)\right) \sinh \theta, \\
R[\gamma] \sqrt{|\gamma|} & =\left(-\frac{2}{L \epsilon}+\mathcal{O}\left(\epsilon^{1}\right)\right) \sinh \theta .
\end{aligned}
$$

Comparing terms of order $\epsilon$ we find that the counter term is:

$$
\int_{\partial M} L_{c t}[\gamma] d^{3} x=\mp \frac{1}{4 \pi L} \int_{\partial M} d^{3} x \sqrt{|\gamma|}\left(1+\frac{L^{2}}{4} R[\gamma]\right) .
$$

By construction, our action is now finite at the boundary $\epsilon \rightarrow 0$ and is of the form:

$$
S_{E}= \pm \frac{\beta \omega}{8 \pi}\left(\frac{t_{h}^{3}}{L^{2}}\right)=\mp \frac{2 \pi L \omega}{8 \pi} \frac{L^{3}}{L^{2}}=\mp \frac{\omega L^{2}}{4} \text {. }
$$

Picking the sign

$$
(r, \theta, \phi) \rightarrow+i(r, \theta, \phi),
$$

for the triple Wick-rotation, the signs of the Euclidean actions agree for both patches, and the actions only differ by the numerical factors $\omega, \omega_{S^{2}}=4 \pi$. As these are numbers, which we could eliminate by taking the Euclidean action per coordinate area, the resulting thermodynamics is the same. 


\section{Planar solutions to the Einstein-Maxwell theory}

Our next examples are vacuum solutions of the Einstein-Maxwell equations with planar symmetry, or 'planar Reissner-Nordström solutions.' These solutions are the simplest examples of a class of planar solutions to the STU model of $\mathcal{N}=2$ supergravity [24], and correspond to the limit where all scalar fields are taken to be constant. It was shown in [24] that planar Einstein-Maxwell solutions already show all the qualitative features of the global causal structure of the full class of solutions. Similarly, we will see in the next section that the thermodynamics of planar Einstein-Maxwell solution is simpler than, but representative of, the thermodynamics of planar solutions of the STU model.

Following our conventions, which are summarized in appendix A, the Lorentzian bulk action for Einstein-Maxwell theory is

$$
S=\frac{1}{16 \pi} \int d^{4} x e\left(-R-F^{\mu \nu} F_{\mu \nu}\right) .
$$

In particular, we work in a convention where Newton's constant is set to unity, $G=1$, so that the gravitational coupling $\kappa_{4}$ satisfies $\kappa_{4}^{2}=8 \pi$. For later use we observe that the Maxwell term has the coefficient $(16 \pi)^{-1}=\left(4 g^{2}\right)^{-1}$, where $g=\sqrt{4 \pi}$ is interpreted as a coupling constant, see appendix C.

\subsection{Static patch}

Solving the Einstein-Maxwell equations while imposing planar symmetry and staticity leads to a Ricci flat solution with the line element

$$
d s^{2}=-f(r) d t^{2}+\frac{d r^{2}}{f(r)}+r^{2}\left(d x^{2}+d y^{2}\right), \quad f(r)=-\frac{2 M}{r}+\frac{q^{2}}{r^{2}},
$$

where we must choose $M>0$ in order to ensure the presence of a horizon. ${ }^{10}$ The transverse coordinate $r$ takes values in the interval $0<r<r_{h}$, where $r=0$ is the location of a curvature singularity, while $r_{h}$ is the location of a Killing horizon, where $f\left(r_{h}\right)=0$. Since we assume that the solution only carries electric charge, the gauge field is given by

$$
F=\left(-\frac{q}{r}\right) d t \wedge d r
$$

The gauge potential $A$ is found through integration of (4.2) together with the standard boundary condition $A\left(r_{h}\right)=0:^{11}$

$$
A=\left(-\frac{q}{r}+\frac{q}{r_{h}}\right) d t
$$

Charge \& chemical potential. The chemical potential is given by the asymptotic value of the gauge potential [40]; taking this limit for (4.3) gives

$$
\mu:=\lim _{r \rightarrow \infty} A_{t}=\frac{q}{r_{h}}=\frac{2 M}{q} .
$$

\footnotetext{
${ }^{10}$ Solutions with $M<0$ have naked singularities.

${ }^{11}$ See [39] appendix $\mathrm{F}$ for an explanation.
} 
Note that while $r \rightarrow \infty$ is outside the static patch $0<r<r_{h}$, we will see below that we can analytically extend spacetime to $0<r<\infty$, so that this limit makes sense. The conserved electric charge is computed using Gauss' law, which for planar symmetry gives

$$
\mathcal{Q}=\frac{1}{4 \pi} \int_{\mathbb{R}^{2}} \star F=\frac{q \omega}{4 \pi}, \quad \omega=\int_{\mathbb{R}^{2}} d x \wedge d y
$$

Here $\omega$ is the divergent parametric area of the horizon, which we keep as a formal constant to allow comparison to the spherically symmetric case. The factor of $4 \pi$ is due to the normalization we have chosen for the gauge field. In our conventions the volume form is defined using the conventional choice $\epsilon_{\text {trxy }}=1$.

\subsection{Dynamic patch}

Due to the presence of a curvature singularity at $r=0$ we cannot apply the standard thermodynamic formalism in the static patch. By analytic continuation, using advanced Eddington-Finkelstein coordinates at an intermediate step, see appendix D, we can extend space time to the dynamical region $r_{h}<r<\infty$, where the horizontal Killing vector field becomes spacelike. Since $r$ becomes a timelike coordinate in the dynamic patch, we apply the same convention as in the de Sitter example, and as in [24], we relabel the coordinates $(t, r) \rightarrow(r, t)$, and redefine $f$ by a minus sign. Then the line element of the dynamic patch takes the form

$$
d s^{2}=-\frac{d t^{2}}{f(t)}+f(t) d r^{2}+t^{2}\left(d x^{2}+d y^{2}\right) \quad f(t)=\frac{2 M}{t}-\frac{q^{2}}{t^{2}} \quad t_{h}=\frac{q^{2}}{2 M}
$$

which covers region III of figure 2 , with $t \rightarrow \infty$ corresponding to past timelike infinity. It has been shown in [24] that this line element becomes asymptotic to a Kasner cosmological solution in the limit $t \rightarrow \infty$. Using advanced Eddington-Finkelstein coordinates, one can show that the Killing horizon between regions III and IV (and I) is an apparent horizon of future inner type, consistent with the interpretation as a contracting cosmological solution, see appendix D.

Temperature \& entropy. To compute the surface gravity and temperature of the future inner horizon, we use the Kodama-Hayward formalism. Applying (2.8) to the line element (4.4), and taking into account that for future horizons the surface gravity and temperature have the same sign we obtain

$$
\kappa=-\frac{4 M^{3}}{q^{4}} \quad \Rightarrow \quad T_{H}=\frac{\kappa}{2 \pi}=-\frac{2 M^{3}}{\pi q^{4}} .
$$

The Bekenstein-Hawking area law gives a relation for the entropy of the horizon in terms of the horizon area:

$$
S_{\mathrm{BH}}=\frac{A}{4}=\frac{\omega t_{h}^{2}}{4}=\frac{q^{4} \omega}{16 M^{2}} .
$$

As with other extensive quantities, we keep the divergent volume $\omega$ as a formal constant rather than using densities. 


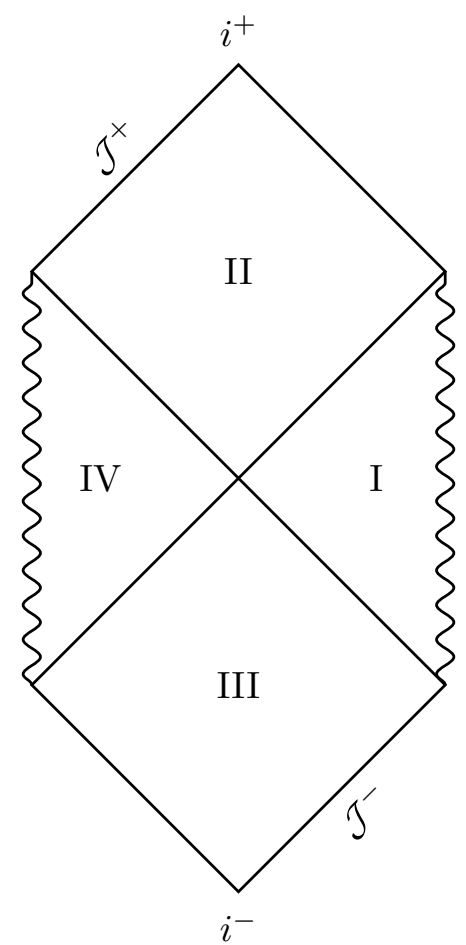

Figure 2. Conformal diagram of the planar Reissner-Nordström solution and planar solution of the STU model. The standard static region, where the Killing vector field $\partial_{t}$ is timelike and futurepointing is Region IV. For thermodynamics we consider the future inner horizon between Regions III and IV, which can be crossed by causal geodesics from the outside to the inside.

\subsection{Euclidean action}

Our main goal is to show that the future inner horizon satisfies the first law of horizon mechanics, which takes the same form as the first law of thermodynamics. This requires the identification of geometrically defined quantities of the solution with thermodynamic quantities. In standard black hole thermodynamics the mass $M$ of the black hole is identified with the internal energy of a canonical or grand canonical ensemble. Due to the planar symmetry, and since we are not working in a static patch, we do not have a natural candidate for a mass-like quantity. Since there is no asymptotically flat region which we could use to normalize the mass or the horizontal timelike Killing vector field, we cannot apply the ADM or Komar approach. We will trade this problem for the one of obtaining a well behaved Euclidean action which we interpret as a grand canonical partition function. The mass-like quantity we identify with the internal energy is then obtained using standard thermodynamic relations. The remaining problem in defining the Euclidean action is its normalisation. For solutions which are asymptotic to a 'vacuum', that is to a maximally symmetric spacetime, the normalisation is fixed by adding a boundary term such that the Euclidean action is zero when evaluated on the vacuum solution. We do not have this option since our solution is not asymptotic to a maximally symmetric spacetime. Moreover, the GHY-boundary term will turn out to be finite, so there is no need to add counterterms. 
However, the integral over the two planar directions is divergent, and while we can formally absorb this in a constant $\omega$, we will allow for a finite multiplicative factor $\mathcal{N}$ between the Euclidean action $S_{E}$ and the grand potential $\Omega$ :

$$
\beta \Omega=\mathcal{N} S_{E}
$$

The constant $\mathcal{N}$ parametrizes the relative normalisation between thermodynamic and geometric quantities. To fix it we can impose one relation, which we choose to be Gauss' law. That is, we identify the charge $\mathcal{Q}$ defined by the gauge field of our field configuration with the negative derivative of $\Omega$ with respect to the chemical potential

$$
\frac{\partial \Omega}{\partial \mu} \stackrel{!}{=}-\mathcal{Q} .
$$

Once $\mathcal{N}$ has been fixed by this condition, all thermodynamic relations must take their standard form, if our interpretation of $\mathcal{Z}=\exp \left(-\mathcal{N} S_{E}\right)$ as a thermodynamic partition function is correct.

Performing the triple Wick-rotation

$$
(r, x, y) \rightarrow \pm i(r, x, y)
$$

we obtain the negative definite line element

$$
d s^{2}=-f(t)^{-1} d t^{2}-f(t) d r^{2}-t^{2}\left(d x^{2}+d y^{2}\right) .
$$

The Euclidean action is given by

$$
\begin{aligned}
S_{E}= & \pm \frac{1}{16 \pi} \int_{M} \sqrt{g} R d^{4} x \pm \frac{1}{8 \pi} \int_{\partial M} \sqrt{|\gamma|} K d^{3} x \\
& \mp \frac{1}{8 \pi} \int_{\partial M} F^{\mu \nu} A_{\mu} d \Sigma_{\nu} .
\end{aligned}
$$

The bulk gauge field term has been transformed into a boundary term. Since the planar Einstein-Maxwell solution has a vanishing Ricci scalar, $R=0$, the action is completely determined by the boundary terms, which are evaluated in the limit where $t \rightarrow \infty$. We do not include a background boundary term, because $S_{E}$ will turn out to be finite.

The hypersurface $\Sigma=\partial M$ is obtained as the limit of a sequence of slices of the spacetime $M$ for constant time $t_{0}$, and has an extrinsic curvature with trace $K$ when considered as an embedded submanifold of $M$. The GHY-term is determined by $K$ and by the induced boundary metric $\gamma[8]$. It can be computed using the formulas reviewed in appendix B with the result

$$
K=\frac{3 M t_{0}-q^{2}}{t_{0}^{2} \sqrt{2 M t_{0}-q^{2}}}, \quad \sqrt{|\gamma|}=t_{0}^{2} \sqrt{\frac{2 M}{t_{0}}-\frac{q^{2}}{t_{0}^{2}}} .
$$

Evaluating this in the limit $t_{0} \rightarrow \infty$ gives

$$
S_{G H Y}= \pm \frac{1}{8 \pi} \int_{\partial M} \sqrt{|\gamma|} K= \pm \frac{3 M \beta \omega}{8 \pi} .
$$


The factor $\beta \omega$ is the parametric volume of the boundary. After Wick-rotation the coordinate $r$ becomes periodic with period $\beta$, in order to avoid a conical singularity at $t=t_{h}{ }^{12}$

As the gauge potential has only one non zero component, the boundary term is simply calculated

$$
\mp \frac{1}{8 \pi} \int_{\partial M} F^{\mu \nu} A_{\mu} d \Sigma_{\nu}= \pm \frac{M \beta \omega}{4 \pi} .
$$

Together, the GHY-term and the gauge field contribution yield the Euclidean action

$$
S_{E}= \pm 5 \frac{M \beta \omega}{8 \pi}
$$

Formally equating the partition function calculated from the Euclidean action with the negative logarithm of the thermal partition function, $\log (\mathcal{Z})=-\mathcal{N} S_{E}=-\beta \Omega$, yields the grand potential

$$
\Omega(\beta, \mu)=\frac{\mathcal{N} S_{E}}{\beta}=\mp 5 \mathcal{N} \frac{\beta \mu^{4} \omega}{(8 \pi)^{2}},
$$

which we have written in terms of its natural thermodynamic variables ${ }^{13} \beta=1 / T$ and $\mu$ using that

$$
M=-\frac{\beta \mu^{4}}{8 \pi}, \quad \mathcal{Q}=-\frac{\mu^{3} \beta \omega}{(4 \pi)^{2}} .
$$

We now apply our normalisation condition (4.7): the conserved charge $\mathcal{Q}$ calculated from Gauss' law must match the negative $\mu$-derivative of $\Omega$. This fixes $\mathcal{N}=\mp \frac{1}{5}$ so that the grand potential is determined to be

$$
\Omega(\beta, \mu)=\frac{\beta \mu^{4} \omega}{(8 \pi)^{2}}
$$

The free energy $F(\beta, \mathcal{Q})$ is obtained as the Legendre transform of the grand potential

$$
F(\beta, \mathcal{Q})=\Omega-\mu \frac{\partial \Omega}{\partial \mu}=\Omega+\mu \mathcal{Q}=3\left(-\frac{\pi^{2} \mathcal{Q}^{4}}{4 \beta \omega}\right)^{\frac{1}{3}}
$$

where we have used the relation

$$
\mu=\left(-\frac{16 \pi^{2} \mathcal{Q}}{\omega \beta}\right)^{1 / 3}
$$

to express the free energy in terms of its natural variables $\beta$ and $\mathcal{Q}$. From $F$ we can compute the thermodynamic entropy $S$ and check that it matches the Bekenstein-Hawking entropy $S_{\mathrm{BH}}$ :

$$
S=\beta^{2} \frac{\partial F}{\partial \beta}=\left(\frac{\pi^{2} \mathcal{Q}^{4} \beta^{2}}{4 \omega}\right)^{\frac{1}{3}}=S_{\mathrm{BH}}
$$

\footnotetext{
${ }^{12}$ To be precise, the conical method determines the period up to sign, and we choose $\beta$ to have the sign determined by the Kodama-Hayward method.

${ }^{13}$ The thermodynamic identities used here and in the following have been summarized in appendix E.
} 
As a further consistency check, we can also verify that the free energy gives us the correct chemical potential:

$$
\frac{\partial F}{\partial \mathcal{Q}}=\left(-\frac{16 \pi^{2} \mathcal{Q}}{\beta \omega}\right)^{1 / 3}=\mu
$$

The internal energy $E$, for which we do not have a geometric definition, is computed using the free energy:

$$
E=\frac{\partial(F \beta)}{\partial \beta}=\left(-\frac{2 \pi^{2} \mathcal{Q}^{4}}{\beta \omega}\right)^{1 / 3}=\frac{M \omega}{4 \pi} .
$$

We observe that $E$ is proportional to the parameter $M$, and therefore $E$ is positive.

Using our previous results we can verify that the thermodynamic variables $E, T, S, \mu, \mathcal{Q}$ satisfy the Smarr relation

$$
E=2 T S+\mu \mathcal{Q} .
$$

Expressing the internal energy $E$ in terms of its natural variables $S$ and $\mathcal{Q}$ we obtain the equation of state

$$
E(S, \mathcal{Q})=\frac{\pi \mathcal{Q}^{2}}{(S \omega)^{1 / 2}}
$$

The partial derivates of the internal energy are

$$
\frac{\partial E}{\partial S}=-\frac{\pi \mathcal{Q}^{2}}{2 S^{3 / 2} \omega^{1 / 2}}=\frac{1}{\beta}=T, \quad \frac{\partial E}{\partial \mathcal{Q}}=\frac{2 \pi \mathcal{Q}}{(S \omega)^{1 / 2}}=\mu
$$

where both expressions have been simplified by substituting in $S(\beta, \mathcal{Q})$ using (4.12). The variation of the internal energy is

$$
d E=\frac{\partial E}{\partial S} d S+\frac{\partial E}{\partial \mathcal{Q}} d \mathcal{Q}=T d S+\mu d \mathcal{Q} .
$$

This relation takes the standard form of the first law of thermodynamics. Note that this works because we have allowed that the temperature is negative. If we had insisted that the temperature is positive, this would have resulted in a non-standard sign for the entropy term.

\section{Planar solutions to the STU model}

We are now in a position to turn to our main application, the planar cosmological solutions of the STU model found in [24], for which we will establish thermodynamical relations, including the first law. The general bosonic Lagrangian for $n$ vector multiplets coupled to $\mathcal{N}=2$ supergravity is

$$
e_{4}^{-1} \mathcal{L}=-\frac{1}{2 \kappa_{4}^{2}} R-\frac{1}{\kappa_{4}^{2}} g_{A \bar{B}} \partial_{\mu} z^{A} \partial^{\mu} \bar{z}^{B}+\frac{1}{4 \kappa_{4}^{2}} \mathcal{I}_{I J} F_{\mu \nu}^{I} F^{J \mid \mu \nu}+\frac{1}{4 \kappa_{4}^{2}} \mathcal{R}_{I J} F_{\mu \nu}^{I} \star F^{J \mid \mu \nu},
$$

where compared to [24] we have restored the four-dimensional gravitational coupling $\kappa_{4}$, see for example [41]. While we used standard supergravity conventions where $\kappa_{4}^{2}=1$ in [24], it will be more convenient in the following to use relativist's conventions where 
$G=1$ and $\kappa_{4}^{2}=8 \pi$, in order to avoid non-standard numerical factors in thermodynamic relations. The couplings $g_{A \bar{B}}, \mathcal{I}_{I J}$ and $\mathcal{R}_{I J}$, where $A, B=1, \ldots, n$ and $I, J=0, \ldots, n$ are functions of the scalar fields $z^{A}$ which can be expressed in terms of a holomorphic function, called the prepotential. The STU model has three vector multiplets, and therefore there are three complex scalars $z^{A}$ and four gauge fields $F_{\mu \nu}^{I}$, including the graviphoton which belongs to the Poincaré supergravity multiplet. The Hodge dual gauge fields are denoted $\star F_{\mu \nu}^{I}$. We refer to [24] for details and only review the results which are directly relevant for the following calculations.

\subsection{Dynamic patch}

The line element in the dynamical patch of the planar symmetric cosmological solution is

$$
d s^{2}=-\frac{\mathcal{H}(\zeta)}{\mathcal{W}(\zeta)} d \zeta^{2}+\frac{\mathcal{W}(\zeta)}{\mathcal{H}(\zeta)} d \eta^{2}+G(\zeta)\left(d x^{2}+d y^{2}\right),
$$

where all functions depend only on the timelike coordinate $\zeta$ :

$$
\begin{aligned}
\mathcal{W}(\zeta) & =\alpha \zeta-1 \\
\mathcal{H}_{a}(\zeta) & =\left(\beta_{a}+\gamma_{a} \zeta\right) \\
\mathcal{H}(\zeta) & =2\left(\mathcal{H}_{0} \mathcal{H}_{1} \mathcal{H}_{2} \mathcal{H}_{3}\right)^{\frac{1}{2}} \\
G(\zeta) & =\zeta^{2}\left[\left(1+\frac{\beta_{0}}{\gamma_{0} \zeta}\right)\left(1+\frac{\beta_{1}}{\gamma_{1} \zeta}\right)\left(1+\frac{\beta_{2}}{\gamma_{2} \zeta}\right)\left(1+\frac{\beta_{3}}{\gamma_{3} \zeta}\right)\right]^{\frac{1}{2}} .
\end{aligned}
$$

Compared to [24] we have performed a rescaling $(\bar{x}, \bar{y}) \mapsto(x, y)$ of the coordinates of the plane which changes the corresponding part of the line element as follows

$$
\begin{aligned}
\mathcal{H}(\zeta)\left(d \bar{x}^{2}+d \bar{y}^{2}\right) & =2 \sqrt{\gamma_{0} \gamma_{1} \gamma_{2} \gamma_{3}} G(\zeta)\left(d \bar{x}^{2}+d \bar{y}^{2}\right), \\
& =G(\zeta)\left(d x^{2}+d y^{2}\right) .
\end{aligned}
$$

In our new parametrization the asymptotic form of the planar line element is $d s_{2}^{2}=$ $\zeta^{2}\left(d x^{2}+d y^{2}\right)$

The integration constants $\beta_{a}, \gamma_{a}$ are related to the integration constants found in the solution [24] via the relations:

$$
\begin{aligned}
& \beta_{a}=\frac{2 K_{a}}{\alpha} \sinh \left(\frac{\alpha h_{a}}{2 K_{a}}\right), \\
& \gamma_{a}=K_{a} \exp \left(-\frac{\alpha h_{a}}{2 K_{a}}\right),
\end{aligned}
$$

where

$$
K_{a}=\left(Q_{0}, P^{1}, P^{2}, P^{3}\right),
$$

are the four non-zero charges carried by the gauge fields $F_{\mu \nu}^{I}$. To avoid a proliferation of cases, we have chosen $Q_{0}, P^{A}$ to be positive. ${ }^{14}$ While $Q_{0}$ is an electric charge, $P^{A}$,

\footnotetext{
${ }^{14}$ Otherwise we would need to distinguish several cases, and to carry along \pm signs, which would be cumbersome without contributing any insights. See [24, 25, 42] for a more detailed discussion.
} 
$A=1,2,3$ are magnetic charges. Explicit formulae for the gauge fields will be given below. The scalar fields are expressed as functions of $\zeta$ through

$$
z^{1}=-i\left(\frac{\mathcal{H}_{0} \mathcal{H}_{1}}{\mathcal{H}_{2} \mathcal{H}_{3}}\right)^{\frac{1}{2}}, \quad z^{2}=-i\left(\frac{\mathcal{H}_{0} \mathcal{H}_{2}}{\mathcal{H}_{1} \mathcal{H}_{3}}\right)^{\frac{1}{2}}, \quad z^{3}=-i\left(\frac{\mathcal{H}_{0} \mathcal{H}_{3}}{\mathcal{H}_{1} \mathcal{H}_{2}}\right)^{\frac{1}{2}}
$$

Having reviewed the planar cosmological solution of [24] we now apply the same procedure as for planar solutions of Einstein-Maxwell theory. The metric (5.2) has a future inner horizon at $\zeta=\zeta_{h}=\alpha^{-1}$ and is asymptotic to a Kasner solution for $\zeta \rightarrow \infty$.

Temperature. Using the Kodama-Hayward formulation, we find that the temperature associated with the future inner horizon is negative and of the form:

$$
\begin{aligned}
T_{H} & =-\left.\frac{1}{4 \pi} \partial_{\zeta}\left(\frac{W(\zeta)}{\mathcal{H}(\zeta)}\right)\right|_{\zeta=\alpha^{-1}} \\
& =-\frac{\alpha^{3}}{8 \pi}\left[\left(\alpha \beta_{0}+\gamma_{0}\right)\left(\alpha \beta_{1}+\gamma_{1}\right)\left(\alpha \beta_{2}+\gamma_{2}\right)\left(\alpha \beta_{3}+\gamma_{3}\right)\right]^{-\frac{1}{2}} .
\end{aligned}
$$

We can simplify this by noting:

$$
\left(\alpha \beta_{a}+\gamma_{a}\right)=K_{a} \exp \left(\frac{\alpha h_{a}}{2 K_{a}}\right)=\frac{K_{a}^{2}}{\gamma_{a}} \quad \Rightarrow \quad T_{H}=-\frac{\alpha^{3}}{8 \pi} \frac{\sqrt{\gamma_{0} \gamma_{1} \gamma_{2} \gamma_{3}}}{Q_{0} P^{1} P^{2} P^{3}} .
$$

Entropy. Using the Bekenstein-Hawking area law we can compute the entropy of the solution:

$$
\begin{aligned}
S_{\mathrm{BH}} & =\frac{G\left(\zeta_{h}\right)}{4}=\frac{1}{4 \alpha^{2}} \exp \left[\frac{\alpha}{2}\left(\frac{h_{0}}{Q_{0}}+\frac{h_{1}}{P^{1}}+\frac{h_{2}}{P^{2}}+\frac{h_{3}}{P^{3}}\right)\right], \\
& =\frac{1}{4 \alpha^{2}} \frac{Q_{0} P^{1} P^{2} P^{3}}{\gamma_{0} \gamma_{1} \gamma_{2} \gamma_{3}} .
\end{aligned}
$$

Since the planar STU solution has several integration constants, we will suppress the parametric volume $\omega$ of the planar directions in this section by setting $\omega=1$. This can be interpreted as either working with densities of divergent extensive quantities, or as compactitfying the planar dimensions on a two-torus.

Chemical potentials. The solution for the gauge field is [24]:

$$
F_{\zeta \eta}^{0}=\left(\dot{A}^{0}\right)_{\eta}=-\frac{Q_{0}}{2\left(\beta_{0}+\gamma_{0} \zeta\right)^{2}}, \quad \tilde{F}_{A \mid \zeta \eta}=\left(\dot{\tilde{A}}_{A}\right)_{\eta}=\frac{P^{A}}{2\left(\beta_{A}+\gamma_{A} \zeta\right)^{2}} .
$$

Here $\tilde{F}_{A \mid \mu \nu}$ denote the duals of the gauge field $F_{\mu \nu}^{A}$. Since the gauge couplings are field dependent, dualisation is not just Hodge dualisation, but involves inverting the couplings. We refer to appendix $\mathrm{C}$ for details. As shown there, the precise relation between gauge fields and dual gauge fields is

$$
\tilde{F}_{A}=-\star \mathcal{I}_{A B} F^{B} \quad \Rightarrow \quad F^{A}=\star \mathcal{I}^{A B} \tilde{F}_{B},
$$

where $F^{A}, \tilde{F}_{A}$ are the two-forms corresponding to the gauge fields, and where $\star$ is the Hodge- $\star$ operator. Note that the coupling matrix $\mathcal{I}_{I J}$ is invertible, and in our convention 
is negative definite. The advantage of using the fields $F^{0}, \tilde{F}_{A}$ instead of $F^{0}, F^{A}$ is that now all gauge fields and charges appearing in the solution are electric. ${ }^{15}$ The corresponding gauge potentials are found by integration, subject to the standard boundary condition $A\left(\zeta_{h}\right)=\tilde{A}\left(\zeta_{h}\right)=0$ :

$$
\left(A^{0}\right)_{\eta}=-\frac{\gamma_{0}(\alpha \zeta-1)}{2 Q_{0}\left(\beta_{0}+\gamma_{0} \zeta\right)}, \quad\left(\tilde{A}_{A}\right)_{\eta}=\frac{\gamma_{A}(\alpha \zeta-1)}{2 P^{A}\left(\beta_{A}+\gamma_{A} \zeta\right)}
$$

We then take the asymptotic limit of the gauge potentials to obtain the chemical potentials

$$
\mu^{0}:=\lim _{\zeta \rightarrow \infty} A_{\eta}^{0}=-\frac{\alpha}{2 Q_{0}}, \quad \tilde{\mu}_{A}:=\lim _{\zeta \rightarrow \infty} \tilde{A}_{A \mid \eta}=\frac{\alpha}{2 P^{A}} .
$$

Electromagnetic charges. As with the Einstein-Maxwell solution, the conserved charges are computed using Gauss' law. However, we need to take into account that the gauge couplings depend on the scalar fields. The gauge field couplings come from $\mathcal{I}_{I J}$ and were calculated explicitly in [24]

$$
\mathcal{I}_{I J}=\operatorname{diag}\left(-s t u,-\frac{t u}{s},-\frac{s u}{t},-\frac{s t}{u}\right), \quad \mathcal{I}^{I J}=\operatorname{diag}\left(-\frac{1}{s t u},-\frac{s}{t u},-\frac{t}{s u},-\frac{u}{s t}\right),
$$

where

$$
s=-\operatorname{Im}\left(z^{1}\right), \quad t=-\operatorname{Im}\left(z^{2}\right), \quad u=-\operatorname{Im}\left(z^{3}\right) .
$$

Putting in the solution (5.3) for the scalar fields $z^{A}$ we can write these couplings as:

$$
\begin{array}{ll}
\mathcal{I}_{00}=-\left(\frac{\mathcal{H}_{0}^{3}}{\mathcal{H}_{1} \mathcal{H}_{2} \mathcal{H}_{3}}\right)^{\frac{1}{2}}, & \mathcal{I}_{11}=-\left(\frac{\mathcal{H}_{0} \mathcal{H}_{2} \mathcal{H}_{3}}{\mathcal{H}_{1}^{3}}\right)^{\frac{1}{2}}, \\
\mathcal{I}_{22}=-\left(\frac{\mathcal{H}_{0} \mathcal{H}_{1} \mathcal{H}_{3}}{\mathcal{H}_{2}^{3}}\right)^{\frac{1}{2}}, & \mathcal{I}_{33}=-\left(\frac{\mathcal{H}_{0} \mathcal{H}_{1} \mathcal{H}_{2}}{\mathcal{H}_{3}^{3}}\right)^{\frac{1}{2}} .
\end{array}
$$

The charge $\mathcal{Q}_{0}$ carried by the gauge field $F^{0}$ is

$$
\mathcal{Q}_{0}=\lim _{\zeta \rightarrow \infty} \frac{1}{8 \pi} \int \star\left(-\mathcal{I}_{00} F^{0}\right)
$$

We refer to appendix $\mathrm{C}$ for a derivation of the expressions for the charges. Evaluating (5.6) we obtain the conserved charge ${ }^{16}$

$$
\mathcal{Q}_{0}=-\frac{1}{16 \pi} \frac{Q_{0}}{\sqrt{\gamma_{0} \gamma_{1} \gamma_{2} \gamma_{3}}}
$$

We use the normalisation $\epsilon_{\eta \zeta x y}=1$ for the volume form, which is the standard normalisation in the static patch of the solution, where $\eta$ is timelike and $\zeta$ spacelike. Note that the Hodge operator contains a factor of $\zeta^{2}$, so that when we evaluate the integral in the limit $\zeta \rightarrow \infty$ we read out the coefficient of the leading term in the integrand, which is

\footnotetext{
${ }^{15}$ This is for computational simplicity. In [28], the authors show how magnetic and electric black hole solutions are equivalent in the semi-classical approach applied here.

${ }^{16}$ Actually charge density, as we set $\omega=1$.
} 
proportional to $1 / \zeta^{2}$. This is the leading behaviour of the field strength $F^{0}$, while the coupling $\mathcal{I}_{00}$ approaches a constant.

As mentioned, we have dualised the magnetic field strengths $F^{A}$ and instead work with their electric duals $\tilde{F}_{A}$, but we must remember that when we dualise a gauge potential in the Lagrangian the corresponding coupling is inverted. This means the conserved dual electric charges are

$$
\tilde{\mathcal{Q}}^{A}=\lim _{\zeta \rightarrow \infty} \frac{1}{8 \pi} \int \star\left(-\mathcal{I}^{A A} \tilde{F}_{A}\right),
$$

which when evaluated on our solution take the values

$$
\tilde{\mathcal{Q}}^{A}=\frac{1}{16 \pi} \frac{P^{A}}{\sqrt{\gamma_{0} \gamma_{1} \gamma_{2} \gamma_{3}}} .
$$

The dual electric charge $\tilde{\mathcal{Q}}^{A}$ can be related to the magnetic charge of $F^{A}$ by $\tilde{\mathcal{Q}}^{A}=-\mathcal{P}^{A}$. This relationship is expanded upon in appendix C.

\subsection{Euclidean action}

Employing the triple Wick-rotation

$$
(\eta, x, y) \rightarrow \pm i(\eta, x, y)
$$

the Euclidean line element has (negative) definite signature and is of the form:

$$
d s^{2}=-\frac{\mathcal{H}(\zeta)}{\mathcal{W}(\zeta)} d \zeta^{2}-\frac{\mathcal{W}(\zeta)}{\mathcal{H}(\zeta)} d \eta^{2}-G(\zeta)\left(d x^{2}+d y^{2}\right)
$$

As we did with the Einstein-Maxwell solution, we evaluate the Euclidean action on-shell, which allows us to write the gauge contributions as boundary terms

$$
\begin{aligned}
S_{E}= & \pm \frac{1}{16 \pi} \int_{M} \sqrt{g}\left(R+2 g_{A \bar{B}} \partial_{\mu} z^{A} \partial^{\mu} \bar{z}^{B}\right) d^{4} x \\
& \pm \frac{1}{8 \pi} \int_{\partial M} \sqrt{|\gamma|} K d^{3} x \\
& \pm \frac{1}{16 \pi} \int_{\partial M}\left(\mathcal{I}_{00} F^{\mu \nu \mid 0}\right) A_{\mu}^{0} d \Sigma_{\nu} \pm \frac{1}{16 \pi} \int_{\partial M}\left(\mathcal{I}^{A A} \tilde{F}_{A}^{\mu \nu}\right) \tilde{A}_{\mu \mid A} d \Sigma_{\nu}
\end{aligned}
$$

We have performed the dualisation procedure such that we work with a purely electric solution.

Cancellation of bulk terms. As in the much simpler case of Einstein-Maxwell theory, the bulk term does not contribute. This is non-trivial since the Ricci scalar does no longer vanish on-shell, $R \neq 0$. However, the gauge field contribution still is a boundary term, and the scalar contribution precisely cancels the gravitational term in the bulk. The trace of Einstein's equation gives that

$$
R_{\mu \nu}-\frac{1}{2} g_{\mu \nu} R=-8 \pi T_{\mu \nu} \quad \Rightarrow \quad R=8 \pi T
$$


In four dimensions the gauge fields do not contribute to the trace of the energy momentum tensor, which therefore is completely given by the scalars:

$$
T=g^{\mu \nu} T_{\mu \nu}=-\frac{2}{8 \pi} g_{A \bar{B}}\left(\partial_{\mu} z^{A} \partial^{\mu} \bar{z}^{B}\right)
$$

which shows that

$$
-\frac{1}{2} R=g_{A \bar{B}}\left(\partial_{\mu} z^{A} \partial^{\mu} \bar{z}^{B}\right),
$$

and therefore the bulk contribution of the solution vanishes. Note that when we set the scalars constant we recover the electro-vac type solution of Einstein-Maxwell theory considered in the previous section, which is not Ricci flat $R_{\mu \nu} \propto T_{\mu \nu} \neq 0$, but has vanishing Ricci scalar.

Calculation of boundary terms. With the bulk terms found vanishing, the Euclidean action for the planar solution of the STU model can be found from the boundary terms. Following the same method as for the planar Einstein-Maxwell solution, the GHY-term is calculated to be

$$
\pm \frac{1}{8 \pi} \int_{\partial M} \sqrt{|\gamma|} K d^{3} x= \pm \frac{3}{32 \pi} \frac{\alpha \beta}{\sqrt{\gamma_{0} \gamma_{1} \gamma_{2} \gamma_{3}}} .
$$

The gauge field term is calculated, through simply substituting in the various components and taking the limit of $\zeta \rightarrow \infty$, obtaining

$$
\pm \frac{1}{16 \pi} \int_{\partial M}\left(\mathcal{I}_{00} F^{\mu \nu \mid 0}\right) A_{\mu}^{0} d \Sigma_{\nu}=\mp \frac{1}{64 \pi} \frac{\alpha \beta}{\sqrt{\gamma_{0} \gamma_{1} \gamma_{2} \gamma_{3}}}
$$

and similarly

$$
\pm \sum_{A=1}^{3} \frac{1}{16 \pi} \int_{\partial M}\left(\mathcal{I}^{A A} \tilde{F}_{A}^{\mu \nu}\right) \tilde{A}_{\mu \mid A} d \Sigma_{\nu}=\mp \frac{3}{64 \pi} \frac{\alpha \beta}{\sqrt{\gamma_{0} \gamma_{1} \gamma_{2} \gamma_{3}}} .
$$

Collecting these terms, the Euclidean action is found to be

$$
S_{E}= \pm \frac{1}{32 \pi} \frac{\alpha \beta}{\sqrt{\gamma_{0} \gamma_{1} \gamma_{2} \gamma_{3}}}
$$

As in the Einstein-Maxwell case we admit a multiplicative constant $\mathcal{N}$ in the relation between the Euclidean action and the grand potential:

$$
\Omega\left(\beta, \mu^{0}, \tilde{\mu}_{A}\right)=\frac{\mathcal{N} S_{E}}{\beta}= \pm \frac{\mathcal{N}}{32 \pi} \frac{\alpha}{\sqrt{\gamma_{0} \gamma_{1} \gamma_{2} \gamma_{3}}} .
$$

The constant $\mathcal{N}$ is fixed by imposing that one of the thermodynamic relations takes its standard form. We choose to impose the relation between the $\mu^{0}$ derivative of $\Omega$ and the charge $\mathcal{Q}_{0}$ :

$$
\left(\frac{\partial \Omega}{\partial \mu^{0}}\right)_{\beta, \tilde{\mu}_{A}} \stackrel{!}{=}-\mathcal{Q}_{0}=\frac{1}{16 \pi} \frac{Q_{0}}{\sqrt{\gamma_{0} \gamma_{1} \gamma_{2} \gamma_{3}}} .
$$

To impose this condition, we first need to express the grand potential $\Omega$ in terms of its natural variables. This can be done using the relationship

$$
\frac{\alpha}{\sqrt{\gamma_{0} \gamma_{1} \gamma_{2} \gamma_{3}}}=\frac{2 \beta}{\pi} \mu^{0} \tilde{\mu}_{1} \tilde{\mu}_{2} \tilde{\mu}_{3}
$$


with the result

$$
\Omega\left(\beta, \mu^{0}, \tilde{\mu}_{A}\right)= \pm \frac{\mathcal{N}}{16 \pi^{2}} \beta \mu^{0} \tilde{\mu}_{1} \tilde{\mu}_{2} \tilde{\mu}_{3} .
$$

Taking the partial derivative we obtain the conserved charge from the grand potential

$$
\left(\frac{\partial \Omega}{\partial \mu^{0}}\right)_{\beta, \tilde{\mu}_{A}}= \pm \frac{\mathcal{N}}{16 \pi^{2}} \beta \tilde{\mu}_{1} \tilde{\mu}_{2} \tilde{\mu}_{3}=\mp \frac{\mathcal{N}}{16 \pi} \frac{Q_{0}}{\sqrt{\gamma_{0} \gamma_{1} \gamma_{2} \gamma_{3}}} .
$$

Comparing this with (5.10) we find that $\mathcal{N}=\mp 1$. This determines the grand potential to be

$$
\Omega\left(\beta, \mu^{0}, \tilde{\mu}_{A}\right)=-\frac{1}{16 \pi^{2}} \beta \mu^{0} \tilde{\mu}_{1} \tilde{\mu}_{2} \tilde{\mu}_{3}=-\frac{1}{8 \pi} \frac{\alpha}{4 \sqrt{\gamma_{0} \gamma_{1} \gamma_{2} \gamma_{3}}} .
$$

Note that $\Omega$ has turned out to be independent of our choice of sign for the triple Wickrotation. It is clear that the other derivatives of $\Omega$ with respect to chemical potentials give the correct corresponding charges.

To obtain the free energy we must Legendre transform the grand potential:

$$
\begin{aligned}
F\left(\beta, \mathcal{Q}_{0}, \tilde{\mathcal{Q}}^{A}\right) & =\Omega+\mu^{0} \mathcal{Q}_{0}+\tilde{\mu}_{1} \tilde{\mathcal{Q}}^{1}+\tilde{\mu}_{2} \tilde{\mathcal{Q}}^{2}+\tilde{\mu}_{3} \tilde{\mathcal{Q}}^{3} \\
& =-\frac{1}{8 \pi} \frac{\alpha}{4 \sqrt{\gamma_{0} \gamma_{1} \gamma_{2} \gamma_{3}}}+\frac{1}{8 \pi} \frac{\alpha}{\sqrt{\gamma_{0} \gamma_{1} \gamma_{2} \gamma_{3}}},
\end{aligned}
$$

and so the free energy is given by:

$$
F\left(\beta, \mathcal{Q}_{0}, \tilde{\mathcal{Q}}^{A}\right)=\frac{1}{8 \pi} \frac{3 \alpha}{4 \sqrt{\gamma_{0} \gamma_{1} \gamma_{2} \gamma_{3}}} .
$$

To express $F$ in terms of its natural thermodynamical variables we use

$$
\beta=-\frac{8 \pi}{\alpha^{3}} \frac{Q_{0} P^{1} P^{2} P^{3}}{\sqrt{\gamma_{0} \gamma_{1} \gamma_{2} \gamma_{3}}} \Rightarrow \frac{\alpha}{\sqrt{\gamma_{0} \gamma_{1} \gamma_{2} \gamma_{3}}}=\left(\frac{(16 \pi)^{5} \mathcal{Q}_{0} \tilde{\mathcal{Q}}^{1} \tilde{\mathcal{Q}}^{2} \tilde{\mathcal{Q}}^{3}}{2 \beta}\right)^{\frac{1}{3}}
$$

and obtain:

$$
F\left(\beta, \mathcal{Q}_{0}, \mathcal{P}^{A}\right)=\frac{3}{32 \pi}\left(\frac{(16 \pi)^{5} \mathcal{Q}_{0} \tilde{\mathcal{Q}}^{1} \tilde{\mathcal{Q}}^{2} \tilde{\mathcal{Q}}^{3}}{2 \beta}\right)^{\frac{1}{3}}
$$

We can now verify that all remaining thermodynamic relations take their standard form. First we verify that the Bekenstein-Hawking entropy matches with the thermodynamic definition:

$$
S=\beta^{2}\left(\frac{\partial F}{\partial \beta}\right)_{\mathcal{Q}_{0}, \tilde{\mathcal{Q}}^{A}}=\frac{1}{4 \alpha^{2}} \frac{Q_{0} P^{1} P^{2} P^{3}}{\gamma_{0} \gamma_{1} \gamma_{2} \gamma_{3}} .
$$

A further consistency check comes from ensuring that the chemical potentials that were found from the gauge field satisfy the standard thermodynamic relations for chemical potentials:

$$
\mu^{0}=\left(\frac{\partial F}{\partial \mathcal{Q}_{0}}\right)_{\beta, \tilde{\mathcal{Q}}^{A}}=\frac{1}{16 \pi \mathcal{Q}_{0}} \frac{\alpha}{2 \sqrt{\gamma_{0} \gamma_{1} \gamma_{2} \gamma_{3}}}=-\frac{\alpha}{2 Q_{0}},
$$

and for the dual gauge fields:

$$
\tilde{\mu}_{A}=\left(\frac{\partial F}{\partial \tilde{\mathcal{Q}}^{A}}\right)_{\beta, \mathcal{Q}_{0}}=\frac{\alpha}{2 P^{A}}
$$


which matches exactly with the chemical potentials found from the asymptotic limit of the vector potentials.

The internal energy of our solution can now be defined by the relation

$$
\begin{aligned}
E=\left(\frac{\partial(\beta F)}{\partial \beta}\right)_{\mathcal{Q}_{0}, \tilde{\mathcal{Q}}^{A}} & =\frac{1}{16 \pi}\left(\frac{(16 \pi)^{5} \mathcal{Q}_{0} \tilde{\mathcal{Q}}^{1} \tilde{\mathcal{Q}}^{2} \tilde{\mathcal{Q}}^{3}}{2 \beta}\right)^{\frac{1}{3}} \\
& =\frac{1}{16 \pi} \frac{\alpha}{\sqrt{\gamma_{0} \gamma_{1} \gamma_{2} \gamma_{3}}} .
\end{aligned}
$$

Next we express the entropy in terms of its natural thermodynamic variables:

$$
S\left(E, \mathcal{Q}_{0}, \tilde{\mathcal{Q}}^{A}\right)=-\frac{16 \cdot 4 \pi^{2} \mathcal{Q}_{0} \tilde{\mathcal{Q}}^{1} \tilde{\mathcal{Q}}^{2} \tilde{\mathcal{Q}}^{3}}{E^{2}}
$$

Note that the entropy is positive, due to $\mathcal{Q}_{0}<0$ and $\mathcal{Q}^{A}>0$, see (5.7) and (5.8), bearing in mind that we we have chosen $Q_{0}$ and $P^{A}$ to be positive. ${ }^{17}$

We need to verify that the Hawking temperature of our solution satisfies the thermodynamic relation

$$
\beta=\frac{1}{T_{H}}=\left(\frac{\partial S}{\partial E}\right)_{\mathcal{Q}_{0}, \mathcal{P}^{A}} .
$$

Taking the partial derivative of $S$ with respect to $E$ we find that

$$
\left(\frac{\partial S}{\partial E}\right)_{\mathcal{Q}_{0} \tilde{\mathcal{Q}}^{A}}=\frac{16 \cdot 8 \pi^{2} \mathcal{Q}_{0} \tilde{\mathcal{Q}}^{1} \tilde{\mathcal{Q}}^{2} \tilde{\mathcal{Q}}^{3}}{E^{3}} .
$$

To compare this with the Hawking temperature we restore the original integration constants:

$$
\left(\frac{\partial S}{\partial E}\right)_{\mathcal{Q}_{0}, \tilde{\mathcal{Q}}^{A}}=-\frac{8 \pi Q_{0} P^{1} P^{2} P^{3}}{\alpha^{3} \sqrt{\gamma_{0} \gamma_{1} \gamma_{2} \gamma_{3}}}=\beta
$$

Thus the Hawking temperature $T_{H}$, calculated from the geometry of the solution agrees with the thermodynamic quantity $T=\partial E / \partial S$.

Smarr relation. Evaluating the grand potential we find

$$
\Omega=E-T S-\mu^{0} \mathcal{Q}_{0}-\tilde{\mu}_{A} \tilde{\mathcal{Q}}^{A}=-\frac{\alpha}{32 \pi \sqrt{\gamma_{0} \gamma_{1} \gamma_{2} \gamma_{3}}}=T S
$$

which we can rearrange in the form of a standard Smarr relation

$$
E=2 T S+\mu^{0} \mathcal{Q}_{0}+\tilde{\mu}_{A} \tilde{\mathcal{Q}}^{A} .
$$

\footnotetext{
${ }^{17}$ Note that the sign of the entropy does not change if we change the signs of all the charges. We have just chosen certain charges to be postive or negative in order to avoid carrying around \pm signs or to distinguish several cases.
} 
First law of thermodynamics. We wish to verify the first law:

$$
d E=T_{H} d S+\mu^{0} d \mathcal{Q}_{0}+\tilde{\mu}_{1} d \tilde{\mathcal{Q}}^{1}+\tilde{\mu}_{2} d \tilde{\mathcal{Q}}^{2}+\tilde{\mu}_{3} d \tilde{\mathcal{Q}}^{3} .
$$

The total differential of $E$ is

$$
d E=\left(\frac{\partial E}{\partial S}\right) d S+\left(\frac{\partial E}{\partial \mathcal{Q}_{0}}\right) d \mathcal{Q}_{0}+\left(\frac{\partial E}{\partial \tilde{\mathcal{Q}}^{1}}\right) d \tilde{\mathcal{Q}}^{1}+\left(\frac{\partial E}{\partial \tilde{\mathcal{Q}}^{2}}\right) d \tilde{\mathcal{Q}}^{2}+\left(\frac{\partial E}{\partial \tilde{\mathcal{Q}}^{3}}\right) d \tilde{\mathcal{Q}}^{3}
$$

Having already found that

$$
\left(\frac{\partial E}{\partial S}\right)=T_{H}
$$

we turn our attention to the derivatives with respect to the charges. Using that:

$$
E^{2}=-\frac{16 \cdot 4 \pi^{2} \mathcal{Q}_{0} \tilde{\mathcal{Q}}^{1} \tilde{\mathcal{Q}}^{2} \tilde{\mathcal{Q}}^{3}}{S}
$$

we find

$$
\left(\frac{\partial E}{\partial \mathcal{Q}_{0}}\right)_{S, \tilde{\mathcal{Q}}^{A}}=-\frac{1}{2 E} \frac{16 \cdot 4 \pi^{2} \tilde{\mathcal{Q}}^{1} \tilde{\mathcal{Q}}^{2} \tilde{\mathcal{Q}}^{3}}{S}=-\frac{\alpha}{2 Q_{0}}=\mu^{0} .
$$

Taking derivatives with respect to the magnetic charges we verify

$$
\left(\frac{\partial E}{\partial \tilde{\mathcal{Q}}^{A}}\right)_{S, \mathcal{Q}_{0}}=\frac{\alpha}{2 P^{A}}=\tilde{\mu}_{A}
$$

Hence we see that the first law of thermodynamics holds.

\section{Comparison to the isolated horizon formalism}

An alternative way of formulating the first law is to work entirely on the horizon. This allows one to calculate thermodynamic variables in the static region of the spacetime where the usual definitions of the thermodynamic constants hold. From [16], we find that the first law using variables defined on the horizon is:

$$
\delta E_{\Delta}=\frac{\kappa \delta a_{\Delta}}{8 \pi G}+\mu_{a} \delta \mathcal{Q}^{a} .
$$

The ambiguity of the energy in the spacetime is fixed by imposing that the infinitesimal energy (mass) is equal to the r.h.s. (6.1). For the remainder of this discussion, we set $G=1$. We note here that this derivation of the first law of black hole mechanics is still only self-consistent as this does not give a direct way to measure the mass outside of the first law itself. The subscript $\Delta$ denotes variables evaluated on the isolated horizon $\Delta$, which for us is the location of our Killing horizon at $\zeta=\alpha^{-1}$. The contracted $a$ index denotes the multiple charges (in our case, we have 4).

\subsection{Planar solutions of the STU model}

Before we begin, we must make a coordinate change into Eddington-Finkelstein coordinates. From there we identify a Killing vector $\ell$ which we use to find $\kappa$. In a similar way to the previous section, we then determine the electromagnetic terms, but this time evaluated on the horizon rather than for $\zeta \rightarrow \infty$. 
Eddington-Finkelstein coordinates. Beginning with the metric from the dynamic region of the spacetime

$$
d s^{2}=-\frac{\mathcal{H}(\zeta)}{\mathcal{W}(\zeta)} d \zeta^{2}+\frac{\mathcal{W}(\zeta)}{\mathcal{H}(\zeta)} d \eta^{2}+G(\zeta)\left(d x^{2}+d y^{2}\right),
$$

we make the coordinate change:

$$
\eta=u+\bar{\zeta}(\zeta), \quad d \eta=d u+\bar{\zeta}^{\prime} d \zeta
$$

which we can substitute into the metric to obtain:

$$
d s^{2}=\left(\frac{\mathcal{W}}{\mathcal{H}}\left(\bar{\zeta}^{\prime}\right)^{2}-\frac{\mathcal{H}}{\mathcal{W}}\right) d \zeta^{2}+\frac{\mathcal{W}}{\mathcal{H}} d u^{2}+\frac{2 \mathcal{W}}{\mathcal{H}} \bar{\zeta}^{\prime} d u d \zeta+G\left(d x^{2}+d y^{2}\right)
$$

By making the choice

$$
\bar{\zeta}^{\prime}=\frac{\mathcal{H}}{\mathcal{W}}
$$

we obtain the EF metric which is well defined for $\zeta=\alpha^{-1}$ :

$$
d s^{2}=\frac{\mathcal{W}}{\mathcal{H}} d u^{2}+2 d u d \bar{\zeta}+G\left(d x^{2}+d y^{2}\right) .
$$

This allows us to identify a suitable null normal vector field

$$
\ell=\frac{\partial}{\partial u} \text {. }
$$

Surface gravity and area term. We can reuse our calculation for the surface gravity from (5.4) to find

$$
\kappa=-\frac{\alpha}{2} \frac{1}{\mathcal{H}\left(\alpha^{-1}\right)} .
$$

From the metric (6.2) we can read off the infinitesimal change in the area as:

$$
\delta a_{\Delta}=\delta\left(G\left(\alpha^{-1}\right)\right)=\delta\left(c \mathcal{H}\left(\alpha^{-1}\right)\right)=\mathcal{H}\left(\alpha^{-1}\right) \delta c+c \delta \mathcal{H}\left(\alpha^{-1}\right), \quad c=\frac{1}{2 \sqrt{\gamma_{0} \gamma_{1} \gamma_{2} \gamma_{3}}},
$$

where as in the Euclidean action formalism we have set

$$
\omega=\int d x \wedge d y=1
$$

for the area of the planar directions.

Putting these together we find that the first term on the r.h.s. of (6.1) is given by:

$$
\kappa \delta a_{\Delta}=-\frac{\alpha}{2}\left(\delta c+\frac{c \delta \mathcal{H}\left(\alpha^{-1}\right)}{\mathcal{H}\left(\alpha^{-1}\right)}\right) .
$$

We can express:

$$
\mathcal{H}\left(\alpha^{-1}\right)=\frac{4 c}{\alpha^{2}} Q_{0} P^{1} P^{2} P^{3}
$$

to find:

$$
\begin{aligned}
\kappa \delta a_{\Delta} & =-\frac{1}{2} \alpha \delta c-\frac{1}{2} \alpha c \delta \log \left(c \alpha^{-2} Q_{0} P^{1} P^{2} P^{3}\right) \\
& =-\alpha \delta c+c \delta \alpha-\frac{1}{2} \alpha c \delta \log \left(Q_{0} P^{1} P^{2} P^{3}\right) .
\end{aligned}
$$


Gauge fields and charges. From the previous calculation we found that the gauge field strengths are given by the relations:

$$
F_{\zeta \eta}^{0}=-\frac{Q_{0}}{2\left(\beta_{0}+\gamma_{0} \zeta\right)^{2}}, \quad \tilde{F}_{A \mid \zeta \eta}=\frac{P^{A}}{2\left(\beta_{A}+\gamma_{A} \zeta\right)^{2}} .
$$

We need to express the gauge field strength in terms of the EF coordinates and then write down the field strength and the correpsonding gauge couplings on the horizon. Starting with the gauge field strengths, we see that they are all of the form:

$$
F=f(\zeta) d \zeta \wedge d \eta
$$

We define a null basis:

$$
\begin{gathered}
d s^{2}=2 e^{+} e^{-}+\delta_{i j} e^{i} e^{j}, \\
e^{+}=d u, \quad e^{-}=d \zeta+\frac{\mathcal{W}}{2 \mathcal{H}} d u, \quad e^{+} \wedge e^{-}=-d \zeta \wedge d u,
\end{gathered}
$$

and we can easily take the Hodge dual:

$$
F=-f(\zeta) e^{+} \wedge e^{-}, \quad \star F=f(\zeta) e^{1} \wedge e^{2}=c \mathcal{H} f(\zeta) d x \wedge d y .
$$

This allows us to write down the Hodge duals explicitly:

$$
\star F^{0}=-\frac{Q_{0}}{2\left(\beta_{0}+\gamma_{0} \zeta\right)^{2}} c \mathcal{H} d x \wedge d y, \quad \star \tilde{F}_{A}=\frac{P^{A}}{2\left(\beta_{A}+\gamma_{A} \zeta\right)^{2}} c \mathcal{H} d x \wedge d y .
$$

Evaluated on the horizon, these gauge fields are:

$$
\star F_{\Delta}^{0}=-\frac{Q_{0} \alpha^{2}}{2\left(\beta_{0} \alpha+\gamma_{0}\right)^{2}} c \mathcal{H}\left(\alpha^{-1}\right) d x \wedge d y, \quad \star \tilde{F}_{A \mid \Delta}=\frac{P^{A} \alpha^{2}}{2\left(\beta_{A} \alpha+\gamma_{A}\right)^{2}} c \mathcal{H}\left(\alpha^{-1}\right) d x \wedge d y .
$$

The last step is to take the gauge couplings (5.5) and evaluate them on the horizon. We find that:

$$
\mathcal{I}_{00 \mid \Delta}=-\frac{2\left(\alpha \beta_{0}+\gamma_{0}\right)^{2}}{\alpha^{2} \mathcal{H}\left(\alpha^{-1}\right)}, \quad \mathcal{I}_{A B \mid \Delta}=-\delta_{A B} \frac{\alpha^{2} \mathcal{H}\left(\alpha^{-1}\right)}{2\left(\alpha \beta_{A}+\gamma_{A}\right)^{2}}
$$

We are now in the position to calculate the conserved charges using the integrals: ${ }^{18}$

$$
\mathcal{Q}_{0 \mid \Delta}=-\frac{1}{8 \pi} \int_{\mathbb{R}^{2}} \star F_{\Delta}^{0} \mathcal{I}_{00 \mid \Delta}, \quad \tilde{\mathcal{Q}}_{\Delta}^{A}=-\frac{1}{8 \pi} \int_{\mathbb{R}^{2}} \star \tilde{F}_{A \mid \Delta} \mathcal{I}_{\Delta}^{A A},
$$

which we can calculate by substituting in the above results to find:

$$
\begin{gathered}
\mathcal{Q}_{0 \mid \Delta}=\left(-\frac{Q_{0} \alpha^{2}}{2\left(\alpha \beta_{0}+\gamma_{0}\right)^{2}} c \mathcal{H}\left(\alpha^{-1}\right)\right) \cdot\left(\frac{2\left(\alpha \beta_{0}+\gamma_{0}\right)^{2}}{\alpha^{2} \mathcal{H}\left(\alpha^{-1}\right)}\right)=-\frac{c Q_{0}}{8 \pi}, \\
\tilde{\mathcal{Q}}_{\Delta}^{A}=\left(\frac{P^{A} \alpha^{2}}{2\left(\alpha \beta_{A}+\gamma_{A}\right)^{2}} c \mathcal{H}\left(\alpha^{-1}\right)\right) \cdot\left(\frac{2\left(\alpha \beta_{A}+\gamma_{A}\right)^{2}}{\alpha^{2} \mathcal{H}\left(\alpha^{-1}\right)}\right)=\frac{c P^{A}}{8 \pi} .
\end{gathered}
$$

\footnotetext{
${ }^{18}$ Note the addition of the extra minus sign as $\mathcal{I}_{I J}<0$, see appendix C.
} 
Gauge potential and chemical potential. In the previous section the gauge fields were found to be:

$$
\left(A^{0}\right)_{\eta}=\frac{Q_{0}}{2 \gamma_{0}\left(\beta_{0}+\gamma_{0} \zeta\right)}, \quad\left(\tilde{A}_{A}\right)_{\eta}=-\frac{P^{A}}{2 \gamma_{A}\left(\beta_{A}+\gamma_{A} \zeta\right)} .
$$

Re-expressing these in terms of the new EF coordinates, evaluated on the horizon, we find that:

$$
\begin{aligned}
A_{\Delta}^{0} & =\frac{Q_{0}}{2 \gamma_{0}} \frac{\alpha}{\alpha \beta_{0}+\gamma_{0}}\left(d u+\frac{\mathcal{H}}{\mathcal{W}} d \zeta\right), \\
\tilde{A}_{A \mid \Delta} & =-\frac{P^{A}}{2 \gamma_{A}} \frac{\alpha}{\alpha \beta_{A}+\gamma_{A}}\left(d u+\frac{\mathcal{H}}{\mathcal{W}} d \zeta\right) .
\end{aligned}
$$

Contracting with the null vector $\ell$ we find the chemical potentials from the identities (this is justified in [16]):

$$
\mu^{0}=-\iota_{\ell} A^{0}, \quad \tilde{\mu}_{A}=-\iota_{\ell} \tilde{A}_{A} .
$$

Simplifying the gauge potential using that

$$
\gamma_{a}\left(\alpha \beta_{a}+\gamma_{a}\right)=K_{a}^{2}
$$

we can write down the chemical potentials:

$$
\mu^{0}=-\frac{\alpha}{2 Q_{0}}, \quad \tilde{\mu}_{A}=\frac{\alpha}{2 P^{A}} .
$$

Now we can write down the second term on the r.h.s. of (6.1) by combining this with the conserved charge from the previous expression to find:

$$
\begin{aligned}
\mu_{a} \delta \mathcal{Q}_{\Delta}^{a} & =\frac{\alpha}{16 \pi}\left(\frac{1}{Q_{0}} \delta\left(c Q_{0}\right)+\frac{1}{P^{1}} \delta\left(c P^{1}\right)+\frac{1}{P^{2}} \delta\left(c P^{2}\right)+\frac{1}{P^{3}} \delta\left(c P^{3}\right)\right) \\
& =\frac{\alpha}{16 \pi}\left(4 \delta c+c \delta \log \left(Q_{0} P^{1} P^{2} P^{3}\right)\right) \\
& =\frac{1}{8 \pi}\left(2 \alpha \delta c+\frac{1}{2} \alpha c \delta \log \left(Q_{0} P^{1} P^{2} P^{3}\right)\right) .
\end{aligned}
$$

First law of black hole mechanics. Using these quantities we are now able to find an expression for the variation of the mass parameter from (6.1). Combining our results we find that:

$$
\begin{aligned}
\frac{\kappa \delta a_{\Delta}}{8 \pi}+\mu_{a} \delta \mathcal{Q}_{\Delta}^{a}= & \frac{1}{8 \pi}\left[-\alpha \delta c+c \delta \alpha-\frac{1}{2} \alpha c \delta \log \left(Q_{0} P^{1} P^{2} P^{3}\right)\right. \\
& \left.+2 \alpha \delta c+\frac{1}{2} \alpha c \delta \log \left(Q_{0} P^{1} P^{2} P^{3}\right)\right] \\
= & \frac{1}{8 \pi}(\alpha \delta c+c \delta \alpha)=\delta\left(\frac{1}{8 \pi} \alpha c\right) \\
= & \delta E_{\Delta}
\end{aligned}
$$

and so:

$$
E_{\Delta}=\frac{\alpha c}{8 \pi}=\frac{\alpha}{16 \pi \sqrt{\gamma_{0} \gamma_{1} \gamma_{2} \gamma_{3}}},
$$

which is identical to the calculation from the Euclidean action. 
Smarr relation. Our last consistency check comes from the Smarr relation we derived in the Euclidean formalism. Taking each piece and summing together we calculate that:

$$
\begin{aligned}
\mu_{a} \mathcal{Q}_{\Delta}^{a}+\frac{\kappa a_{\Delta}}{8 \pi}= & \frac{1}{8 \pi}\left[c Q_{0} \cdot \frac{\alpha}{2 Q_{0}}+c P^{1} \cdot \frac{\alpha}{2 P^{1}}+c P^{2} \cdot \frac{\alpha}{2 P^{2}}\right. \\
& \left.+c P^{3} \cdot \frac{\alpha}{2 P^{3}}-c \mathcal{H}\left(\alpha^{-1}\right) \cdot \frac{\alpha}{2 \mathcal{H}\left(\alpha^{-1}\right)}\right] \\
= & \frac{\alpha c}{16 \pi}(1+1+1+1-1) \\
= & \frac{3}{16 \pi} \alpha c=\frac{3 E_{\Delta}}{2}
\end{aligned}
$$

which is identical to the one from the Euclidean action formalism.

\subsection{Planar solutions to Einstein-Maxwell theory}

Einstein-Maxwell theory is a consistent truncation of the STU model where all four gauge fields are set equal, while the scalar fields are constant. Therefore we can map the solution of the STU model to that of the Einstein-Maxwell model by fine tuning the integration constants. The physical scalar fields are given by

$$
z^{A}=i \mathcal{H}_{A}\left(-\frac{\mathcal{H}_{0}}{\mathcal{H}_{1} \mathcal{H}_{2} \mathcal{H}_{3}}\right)^{\frac{1}{2}}
$$

and we see that they are everywhere constant under the restriction that $\mathcal{H}_{0}=\mathcal{H}_{1}=\mathcal{H}_{2}=$ $\mathcal{H}_{3}$. This means the integration constants must be fine-tuned, such that $Q_{0}=P^{1}=$ $P^{2}=P^{3}=K$ and $h_{0}=h^{1}=h^{2}=h^{3}=h$. Consequently the four gauge fields take identical values, and the degrees of freedom contributing to the solution match those of Einstein-Maxwell theory. Following this through, the integration constants have the form

$$
\alpha=2 B, \quad \beta=\frac{2 K}{\alpha} \sinh \left(\frac{\alpha h}{2 K}\right), \quad \gamma=\exp \left(-\frac{\alpha h}{2 K}\right), \quad \alpha, \beta, \gamma \in(0, \infty)
$$

in the Einstein-Maxwell limit, and the line element becomes

$$
d s^{2}=-\frac{2(\beta+\gamma \zeta)^{2}}{(\alpha \zeta-1)} d \zeta^{2}+\frac{(\alpha \zeta-1)}{2(\beta+\gamma \zeta)^{2}} d \eta^{2}+\zeta^{2}\left(1+\frac{\beta}{\gamma \zeta}\right)^{2}\left(d x^{2}+d y^{2}\right)
$$

By comparing this with (4.4), we can express the parameters $M, q$ of the Einstein-Maxwell solution in terms of the constrained integration constants of the STU solution:

$$
M=\frac{\alpha}{4 \gamma^{2}}, \quad q^{2}=\frac{\alpha \beta+\gamma}{2 \gamma^{3}}=\frac{K^{2}}{2 \gamma^{4}}
$$

For a full discussion of this mapping, see appendix B of [24].

We can now study each piece of the above calculation and see how it changes under this mapping, and see that we recover the results from the Euclidean action formalism in 
section 4. In the Einstein-Maxwell limit each of the relavant pieces simplifies into the form

$$
\begin{aligned}
\kappa & =-\frac{\alpha^{3} \gamma^{2}}{4 K^{4}}=-\frac{4 M^{3}}{q^{4}}, \quad a_{\Delta}=\frac{K^{4}}{\gamma^{4} \alpha^{2}}=\frac{q^{4}}{4 M^{2}}, \\
\mathcal{Q}_{0 \mid \Delta} & =-\tilde{\mathcal{Q}}_{\Delta}^{A}=-\frac{K}{16 \pi \gamma^{2}}=-\frac{\sqrt{2} q}{16 \pi}, \\
\mu^{0} & =-\tilde{\mu}_{A}=-\frac{\alpha}{2 K}=-\frac{2 M}{\sqrt{2} q},
\end{aligned}
$$

where we have used that $c^{-1}=2 \gamma^{2}$.

Looking at the variation of the appropriate terms:

$$
\begin{aligned}
\kappa \delta a_{\Delta} & =-\frac{4 M^{3}}{q^{4}}\left(\frac{q^{3}}{M^{2}} \delta q-\frac{q^{4}}{2 M^{3}} \delta M\right)=-4 \cdot\left(\frac{M}{q} \delta q-\frac{1}{2} \delta M\right), \\
\mu_{a} \delta \mathcal{Q}^{a} & =4 \cdot\left(\frac{2 M}{\sqrt{2} q} \frac{\sqrt{2}}{16 \pi} \delta q\right)=\frac{M}{2 \pi q} \delta q,
\end{aligned}
$$

we obtain an expression for the variation of the energy by imposing the first law

$$
\delta E_{\Delta}:=\frac{\kappa \delta a_{\Delta}}{8 \pi}+\mu_{a} \delta \mathcal{Q}^{a}=\frac{\delta M}{4 \pi} .
$$

When this is integrated up, we obtain an expression for the internal energy

$$
E_{\Delta}=\frac{M}{4 \pi},
$$

which matches exactly with the internal energy derived from the free energy, via the Euclidean action formalism in section 4 .

\section{Planar solutions to Einstein-anti-Maxwell theory}

In this section, we report on a 'dual version' of the planar Einstein-Maxwell solution, where the static and dynamic regions are exchanged such that the first law and other thermodynamic relations can be derived using a conventional Wick-rotation. The price to be paid for this is to flip the sign of the Maxwell term. This theory is sometimes referred to as Einstein-anti-Maxwell, and in general fields with a flipped sign kinetic terms are referred to as "phantom" fields $[43,44]$. Fields with negative kinetic energy have been discussed in the context of cosmology, because some data suggest that the current expansion of the universe is over-exponential, leading to a 'big-rip' cosmological singularity in finite time. While naively the negative kinetic energy renders the theory unstable, $p$-form gauge fields with inverted kinetic terms appear in the type-II* string theories which are related to type-II string theories by timelike T-duality transformations. In these cases, the theory is made consistent through the presence of massive string modes and the related higher gauge symmetries [26]. Gauge fields with flipped kinetic terms also appear in "Fake-Supergravity" theories. 
We will now show that the Einstein-anti-Maxwell theory admits a planar solution which can be viewed as the 'dual' partner of to the planar cosmological solution of EinsteinMaxwell theory. This solution realizes the same thermodynamical system as studied in section 4, in the sense that both solutions have the same Euclidean action, and therefore the same grand potential and other thermodynamic potentials. More precisely, the range of some of thermodynamic quantities (temperature, energy) will turn out to be different, suggesting that the two solutions represent different 'phases' of the same system. We will discuss the interpretation of these observations in section 8 .

\subsection{The solution}

We start with an action which simultaneously describes both theories,

$$
S=\int d^{4} x \sqrt{-g}\left(-\frac{1}{2 \kappa_{4}^{2}} R+\frac{\varepsilon}{4 g^{2}} F^{2}\right)
$$

where $\varepsilon= \pm 1$ and $g^{2}=4 \pi$ is the gauge coupling. Introducing $g$ is convenient because it allows us to relate both theories by analytic continuation of the coupling constant $g \rightarrow i g$. Alternatively we could relate them by analytic continuation of the gauge fields $F$, but we prefer to keep $F$ real valued in both theories. This said, we revert to our standard conventions where $G=1, \kappa_{4}^{2}=8 \pi$ and $g^{2}=4 \pi$.

Solving Einstein's equations with a static, planar symmetric ansatz yields a line element of the form:

$$
d s^{2}=-f(r) d t^{2}+f(r)^{-1} d r^{2}+r^{2} d \vec{X}^{2}, \quad f(r)=\frac{2 c}{r}+\frac{\varepsilon q^{2}}{r^{2}} .
$$

For spherically symmetric solutions, the value of the integration constant $c$ is set by comparing the result in a weak field limit to Newtonian results. In planar symmetric theories, this is not possible as there is no asymptotically flat region. Instead we choose the sign of $c$ by imposing the existence of a Killing horizon, which implements cosmic censorship by placing the singularity at $r=0$ behind a horizon. With this in mind, we can write the line element as

$$
d s^{2}=-f(r) d t^{2}+f(r)^{-1} d r^{2}+r^{2} d \vec{X}^{2}, \quad f(r)=\varepsilon\left(\frac{2 M}{r}-\frac{q^{2}}{r^{2}}\right),
$$

where the integration constant $M$ is always positive. In this form, it is easy to see that the sign of $f(r)$ is set by $\varepsilon$. Namely, when $\varepsilon=-1$, the asymptotic region is dynamic and the static patch for the solution is a finite region of the spacetime, bounded by

$$
0<r<\frac{q^{2}}{2 M} .
$$

Conversely, for $\varepsilon=1$ the static region is found for coordinate values of

$$
r>\frac{q^{2}}{2 M},
$$

and we see that for Einstein-anti-Maxwell, the asymptotic region of the spacetime is static. Asymptotically this metric is the vacuum Taub solution [45]. This allows the Wick-rotation 
of the timelike coordinate $t$ to produce a (positive) definite, real line element. Unlike the Einstein-Maxwell solution, we have the standard relation between quantum mechanics and statistical mechanics, which identifies the saddle point approximation of the gravitational functional integral with a thermodynamic potential.

In the following, we set $\varepsilon=1$ and calculate the Euclidean action and thermodynamic potentials. The line element in the static region is

$$
d s^{2}=-f(r)^{2} d t^{2}+\frac{d r^{2}}{f(r)}+r^{2} d \vec{X}^{2}, \quad f(r)=\frac{2 M}{r}-\frac{q^{2}}{r^{2}}, \quad r_{h}=\frac{q^{2}}{2 M} .
$$

Chemical potential. The gauge field is

$$
F=\left(-\frac{q}{r}\right) d t \wedge d r, \quad A=\left(-\frac{q}{r}+\frac{q}{r_{h}}\right) d t,
$$

and by taking the asymptotic limit of the gauge potential, we obtain the chemical potential

$$
\mu=\lim _{r \rightarrow \infty} A_{t}=\frac{2 M}{q} .
$$

Conserved charge. The sign flip of the gauge field coupling leads to a sign flip in the conserved charge, as we explain in appendix C. Therefore

$$
\mathcal{Q}=-\frac{1}{4 \pi} \int_{\partial \Sigma} \star F=-\frac{q}{4 \pi} .
$$

Note that we have set $\omega=1$ for simplicity.

Entropy \& Temperature. The Einstein-anti-Maxwell solution has an exterior region with a timelike Killing vector which allows the surface gravity to be calculated by the standard method from the Killing vector field.

$$
\kappa=\frac{4 M^{3}}{q^{4}} \Rightarrow \beta=\frac{\pi q^{4}}{2 M^{3}} .
$$

We remark that using the Kodama-Hayward expression (2.8), we obtain an identical result. We show in appendix D that the horizon separating the static exterior from a dynamic interior is a future outer horizon. In figure 3 these are regions I and II (or regions IV and II) in the conformal diagram on the right hand side. This being a future outer horizon, we have $\kappa \propto T_{H}$ and the temperature is positive, but has the same magnitude as in the Einstein-Maxwell solution.

The Bekenstein-Hawking area law gives

$$
S_{\mathrm{BH}}=\frac{r_{h}^{2}}{4}=\frac{q^{4}}{16 M^{2}} .
$$

\subsection{Euclidean action}

After the Wick-rotation $t \rightarrow-i t$ the Euclidean action is given by (2.2), with the addition of a sign flip for the gauge field contribution

$$
\begin{aligned}
S_{E}= & \frac{1}{16 \pi} \int_{M} \sqrt{g} R d^{4} x \\
& -\frac{1}{8 \pi} \int_{\partial M} \sqrt{|\gamma|} K d^{3} x+\frac{1}{8 \pi} \int_{\partial M} F^{\mu \nu} A_{\mu} d \Sigma_{\nu} .
\end{aligned}
$$



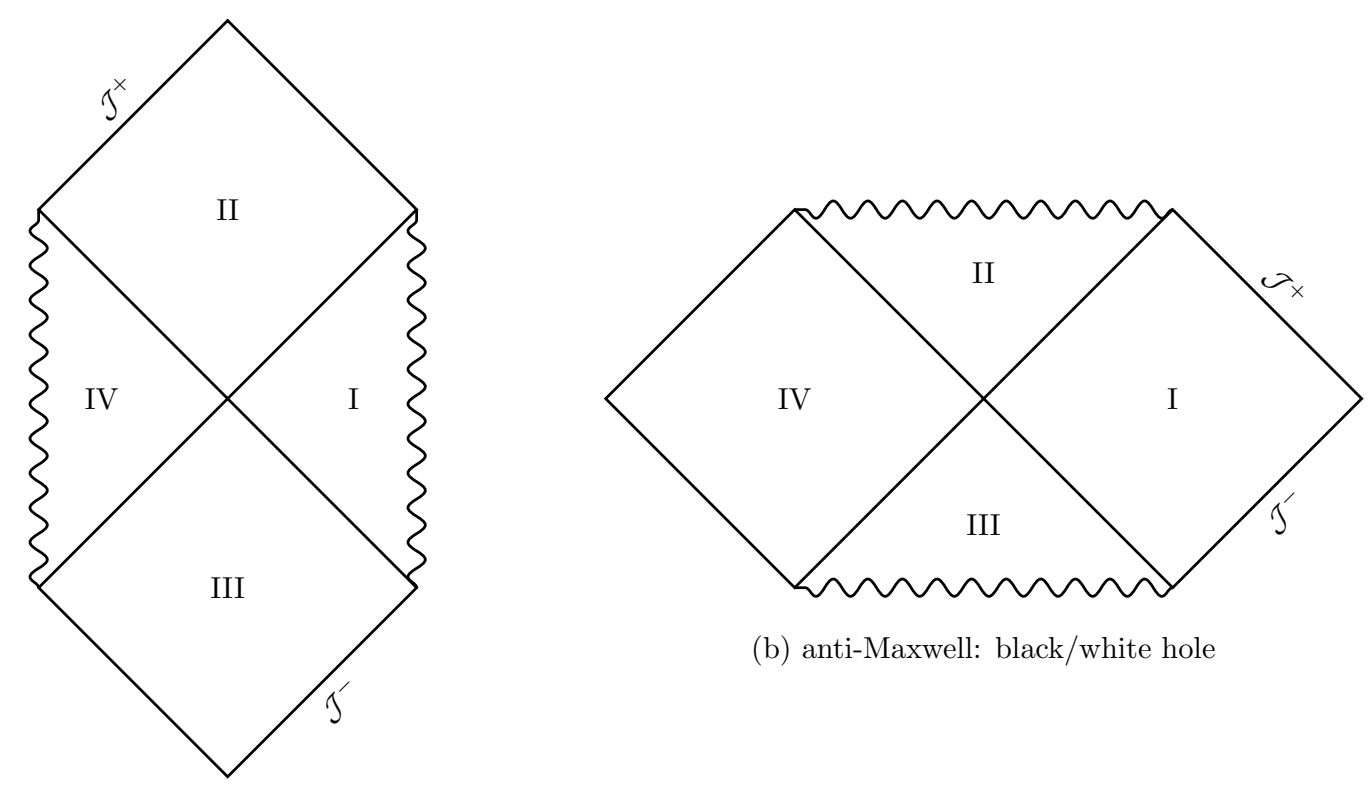

(b) anti-Maxwell: black/white hole

(a) Maxwell: cosmological

Figure 3. Comparison of the conformal diagrams of cosmological and black hole solutions. Left side: Planar cosmological solution of Einstein-Maxwell theory. Right side: Planar black bole solution of Einstein-anti-Maxwell theory, same as for the (spherical) Schwarzschild solution of pure Einstein theory.

The bulk term does not contribute, since $R=0$. The GHY-term is

$$
-\frac{1}{8 \pi} \int_{\partial M} \sqrt{\gamma} K=-\frac{3 M \beta}{8 \pi}
$$

The gauge field contribution is identical to the one of the Einstein-Maxwell solution

$$
\frac{1}{8 \pi} \int_{\partial M} F^{\mu \nu} A_{\mu} d \Sigma_{\nu}=-\frac{2 M \beta}{8 \pi},
$$

and when these two pieces are taken together, the Euclidean action is found to be

$$
S_{E}=-\frac{5 \beta M}{8 \pi}
$$

which is the same the Euclidean action of the triple Wick-rotated Einstein-Maxwell action (4.9).

As the charge is kept fixed, we associate the grand canonical thermodynamic partition function with the saddle-point approximation of the gravitational partition function:

$$
\log \mathcal{Z}=-\mathcal{N} S_{E}=-\beta \Omega \quad \Rightarrow \quad \Omega(\beta, \mu)=-\frac{5 \mathcal{N} \beta \mu^{4}}{(8 \pi)^{2}}
$$


where we have expressed $\Omega$ in terms of its natural thermodynamical variables. The normalisation constant $\mathcal{N}$ is fixed by imposing the relation

$$
\frac{\partial \Omega}{\partial \mu}=-\mathcal{Q} .
$$

Since

$$
\frac{\partial \Omega}{\partial \mu}=-20 \mathcal{N} \frac{\mu^{3} \beta}{(8 \pi)^{2}}, \quad-\mathcal{Q}=\frac{q}{4 \pi}=\frac{\mu^{3} \beta}{(4 \pi)^{2}}, \quad \mu=\left(-\frac{(4 \pi)^{2} \mathcal{Q}}{\beta}\right)^{1 / 3},
$$

this fixes $\mathcal{N}=-\frac{1}{5}$, so that the grand potential for the planar Einstein anti-Maxwell solution is

$$
\Omega(\beta, \mu)=\frac{\beta \mu^{4}}{(8 \pi)^{2}} .
$$

The free energy is then obtained by a Legendre transformation:

$$
F(\beta, \mathcal{Q})=\Omega+\mu \mathcal{Q}=-3\left(\frac{\mathcal{Q}^{4} \pi^{2}}{4 \beta}\right)^{\frac{1}{3}}
$$

By taking partial derivatives we can verify that the following two thermodynamic relations take their standard forms:

$$
\frac{\partial F}{\partial \mathcal{Q}}=\left(-\frac{(4 \pi)^{2} \mathcal{Q}}{\beta}\right)^{\frac{1}{3}}=\mu, \quad S=\beta^{2} \frac{\partial F}{\partial \beta}=\left(\frac{\mathcal{Q}^{4} \beta^{2} \pi^{2}}{4}\right)^{\frac{1}{3}}=S_{\mathrm{BH}} .
$$

Therefore we are confident in defining the internal energy as

$$
E=\frac{\partial(F \beta)}{\partial \beta}=-\frac{M}{4 \pi}<0 .
$$

We note that $E$ is negative, which reflects that in the Einstein-anti Maxwell theory the vector field has negative kinetic energy. By expressing $E$ in terms of its natural thermodynamic variables we obtain the following equation of state:

$$
E(S, \mathcal{Q})=-\frac{\pi \mathcal{Q}^{2}}{\sqrt{S}}
$$

Finally, we compute the total differential of the internal energy,

$$
d E=\frac{\partial E}{\partial S} d S+\frac{\partial E}{\partial \mathcal{Q}} \mathcal{Q}=\beta^{-1} d S+\mu d \mathcal{Q},
$$

and find that the first law is satisfied. It is interesting to note that the Euclidean action and grand potential, as well as other thermodynamic relations, are the same as for the planar solutions of Einstein-Maxwell theory, except for the range of some of the variables. For the Einstein-Maxwell solution temperature is negative and internal energy is positive, while for the Einstein-anti Maxwell solution temperature is positive and internal energy is negative. While both solutions exhibit features indicating instabilities (negative temperature and negative internal energy, respectively), they obey all formal relations of thermodynamics and have the same underlying Euclidean action. 


\section{Discussion and outlook}

In this paper, we have developed a modified version of the Euclidean approach to horizon thermodynamics, which can be applied to a class of cosmological spacetimes whose causal structure is related to black hole solutions by exchanging the role of exterior and interior. By applying a triple Wick-rotation we obtain a finite Euclidean on-shell action which defines a grand thermodynamical potential, from which all thermodynamic quantities, the Smarr relation and the first law can be derived. For planar solutions of Einstein-Maxwell theory and of the STU model, which are asymptotic to Kasner cosmological solutions, the formalism allows the definition of a positive mass-like quantity. The results obtained using the triple Wick-rotation are consistent with those from the isolated horizon formalism, which is another check of its validity. Both approaches are complementary. The isolated horizon formalism is quasi-local, and does not require knowledge of the global spacetime geometry. But it therefore misses out on finding an underlying Euclidean action which defines the grand potential and on how a mass-like quantity is obtained from the thermodynamic formalism. In the isolated horizon formalism, the mass is instead determined locally by imposing that the first law holds. The Euclidean action is also required to make the connection between planar solutions of Einstein-Maxwell and Einstein-anti-Maxwell theory.

For the thermodynamic formalism, we used the future inner horizons of the maximally extended solutions. This is natural because these horizons can be crossed by causal geodesics from the outside to the inside, which is the same situation as for black holes. For future inner horizons the surface gravity and temperature are both negative, when computed according to [33-35], and we have shown that the first law takes its standard form. It is natural to ask what happens if we use the past horizons instead, where causal geodesics cross from the interior to the exterior. This is analogous to asking about the thermodynamics of white holes. For our cosmological solutions the past horizons are past inner horizons. Since the surface gravity is still negative, the expression $\kappa d A$ and hence the 'first law of horizon mechanics' retains its standard form. However, the temperature is now positive and the 'first law of thermodynamics' takes a non-standard form where the sign of the temperature/entropy term is flipped, $T d S \rightarrow-T d S$. This depends, of course, on accepting both the definition of the surface gravity by Kodama-Hayward and the sign of the Hawking temperature being determined by the Parikh-Wilczek tunneling method. We leave the investigation of this observation to future work. Another question which deserves further attention is the interpretation of the negative temperature and, for planar black holes, negative energy. There is also the question how the thermodynamics defined using the triple Wick rotation relates to an underlying microscopic description, given that the 'Hamiltonian' we use is actually related to spatial translations. Finally, one should ask whether one can formulate the thermodynamics of horizons for more general non-static solutions using a variational approach as in [12], modified to imposing initial rather than boundary conditions.

Besides formulating thermodynamics using a non-stationary spacetime patch, we also have uncovered a curious relation or 'duality' between cosmological and black hole solutions, induced by flipping the sign of the Maxwell term, which exchanges interior and exterior, 


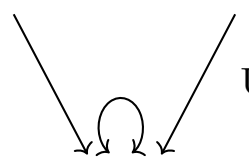

$(0,4)$
$\mathrm{U}(2)$

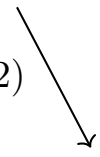

$(1,3)$

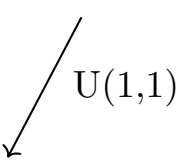

$3)^{2}$

Figure 4. This diagram summarises the relations between five-dimensional and four-dimensional vector multiplet theories with spacetime signature $(t, s)$, that is, $t$ timelike and $s$ spacelike dimensions [27]. The two four-dimensional theories in a given signature differ by relative signs between terms in their Lagrangians. In Euclidean signature, these signs can be changed by a suitable field redefinition, and the Euclidean theory is unique. In Minkowski signature there are two nonisomorphic supersymmetry algebras which are distinguished by their R-symmetry groups $\mathrm{U}(2)$ and $\mathrm{U}(1,1)$, respectively. Therefore the corresponding vector multiplets of these theories cannot be related by a field redefinition.

spacelike and timelike singularities, and which relates solutions with negative temperature to solutions with negative energy. We think that a promising way to better understand these features is the embedding into string theory, to which we turn now. The realisation of a duality between two distinct Lorentzian solutions, via the equivalence of their Euclidean actions, has an interesting relation to recent results in $\mathcal{N}=2$ supersymmetry. In [27] fourdimensional $\mathcal{N}=2$ supersymmetry algebras have been classified for all possible signatures $(t, s)$, where $t$ is the number to timelike and $s$ the number of spacelike dimensions. It was found that while the $\mathcal{N}=2$ supersymmetry algebra is unique in Euclidean signature $(0,4)$, there are two inequivalent algebras in Minkowski signature $(1,3)$, namely the standard algebra with compact R-symmetry group $\mathrm{U}(2)$ and a twisted version with R-symmetry group $\mathrm{U}(1,1)$. The corresponding vector multiplet theories are distinguished by relative signs between various terms in the Lagrangian, including a relative sign between Maxwell and scalar terms. Already in [46] it has been shown that a non-standard $\mathcal{N}=2$ supergravity theory coupled to vector multiplets with inverted signs for all Maxwell-like terms results from dimensional reduction of five-dimensional supergravity coupled to vector multiplets with signature $(2,3)$. This theory reduces to Einstein-anti-Maxwell theory upon truncating out the matter fields and the gravitini. We remark that while vector multiplet theories in signature $(0,4)$ can likewise be obtained in two ways from five dimensions, the resulting relative signs can be removed by a suitable field redefinition, since the underlying Euclidean supersymmetry algebra is unique up to isomorphism [27]. The situation is summarized in figure 4.

The relative sign flips between the Minkowski signature theories are of the same type as those between type-II and type-II* string theory, which are related to each other by timelike T-duality [26]. Moreover, $\mathcal{N}=2$ supergravity with vector (and hyper) multiplets arises by compactification of type-II string theory on Calabi-Yau threefolds. In a future publication we will present the details of the embedding of the twisted $\mathcal{N}=2$ supergravity theory into type-II* theory and show that the STU and anti-STU model (which generalizes the Einstein-anti-Maxwell theory considered in this paper) are related by T-duality [47]. We expect that this will shed more light onto the thermodynamics of planar solutions and 
their microscopic interpretation in terms of string theory. We remark that when combining timelike and spacelike T-duality with S-duality, it is possible to change spacetime signature in type-II string theory, which provides a second way, besides analytical continuation, of relating theories in Euclidean and in Minkowski signature [48]. Solutions in neutral and in general signature have recently found attention in the literature, see for example [49-51].

Both from the point of view of thermodynamics and from the one of T-duality, certain spacetime geometries naturally form pairs which share the same underlying Euclidean description. If one takes the Euclidean functional integral as fundamental and allows both the spacetime and the field space to be complex-valued, this will correspond to pairs of complex saddle points of the functional integral which represent dual Minkowksi signature solutions. At this point it is not clear whether the two dual solutions are actually 'the same', that is, gauge equivalent under a chain of string duality transformations, or just have 'the same thermodynamics.' In either case one could also look for relations to solutions in neutral signature. It will be interesting to further investigate these intriguing relations between geometry, thermodynamics and dualities.

\section{Acknowledgments}

Giacomo would like to thank Alessandro Torrielli, for the enlightening discussions about the physical interpretation of a triple Wick-rotation.

\section{A Conventions}

We use the same parametrization of conventional signs as in [52]. When studying general relativity this involves three conventional sign choices $s_{i}= \pm 1, i=1,2,3$. The first is the overall sign of the Minkowski metric

$$
\eta_{a b}=s_{1} \operatorname{diag}(-+++),
$$

and decides whether we work with a "mostly-plus" or "mostly-minus" signature. The second sign choice comes from the definition of the Riemann tensor:

$$
R_{\nu \rho \sigma}^{\mu}=s_{2}\left(\partial_{\rho} \Gamma_{\nu \sigma}^{\mu}-\partial_{\sigma} \Gamma_{\nu \rho}^{\mu}+\Gamma_{\nu \sigma}^{\tau} \Gamma_{\tau \rho}^{\mu}-\Gamma_{\nu \rho}^{\tau} \Gamma_{\tau \sigma}^{\mu}\right),
$$

and the third sign from the Einstein equations

$$
s_{3}\left(R_{\mu \nu}-\frac{1}{2} g_{\mu \nu} R\right)=\kappa_{4}^{2} T_{\mu \nu},
$$

where it is understood that $T_{00}$ is always positive (for normal matter). The signs $s_{2}, s_{3}$ enter into the definitions of the Ricci tensor and Ricci scalar:

$$
s_{2} s_{3} R_{\mu \nu}=R_{\mu \rho \nu}^{\rho}, \quad R=g^{\mu \nu} R_{\mu \nu} .
$$

These three signs enter into a Lagrangian for gravity, vector and scalar fields as:

$$
\mathcal{L}=\left(s_{1} s_{3} \frac{R}{2 \kappa_{4}^{2}}-s_{1} \frac{1}{\kappa_{4}^{2}} \partial_{\mu} \phi \partial^{\mu} \phi-\frac{1}{4 g^{2}} F_{\mu \nu} F^{\mu \nu}\right) .
$$


The conventions used in a particular paper can usually be reconstructed using that the kinetic terms are positive. This depends of cause on knowing that the overall sign of the Lagrangian has been fixed accordingly, and that we are not dealing with a non-standard theory with flipped kinetic terms. We also need to assume that the energy momentum Tensor is defined such that $T_{00}$ is positive and and therefore:

$$
T_{\mu \nu}=-s_{1} \frac{2}{\sqrt{-g}} \frac{\delta\left(\mathcal{L}_{m} \sqrt{-g}\right)}{\delta g^{\mu \nu}}
$$

where $\mathcal{L}_{m}$ is the matter contribution to the Lagrangian.

In this work we use the same sign conventions as in [24] which in turn were taken over from [42]. This is a parametrization where the Einstein-Hilbert and scalar term enter with a minus sign:

$$
\mathcal{L}=\left(-\frac{R}{2 \kappa_{4}^{2}}-\frac{1}{\kappa_{4}^{2}} \partial_{\mu} \phi \partial^{\mu} \phi-\frac{1}{4 g^{2}} F_{\mu \nu} F^{\mu \nu}\right)
$$

From this we can read off

$$
s_{1}=1, \quad s_{3}=-1 .
$$

Defining the Ricci tensor such that $s_{2} s_{3}=1$, consistency determines the overall sign of the Riemann tensor as $s_{2}=-1$,

$$
R_{\nu \rho \sigma}^{\mu}=-\left(\partial_{\rho} \Gamma_{\nu \sigma}^{\mu}-\partial_{\sigma} \Gamma_{\nu \rho}^{\mu}+\Gamma_{\nu \sigma}^{\tau} \Gamma_{\tau \rho}^{\mu}-\Gamma_{\nu \rho}^{\tau} \Gamma_{\tau \sigma}^{\mu}\right) .
$$

It follows that Einstein's equations are:

$$
R_{\mu \nu}-\frac{1}{2} g_{\mu \nu} R=-\kappa_{4}^{2} T_{\mu \nu}
$$

With these conventions, a spacelike surface of positive curvature has $\operatorname{sign}(R)=s_{1} s_{3}=$ -1 , such that a positively curved space has a negative Ricci scalar. From the perspective of the (anti) de Sitter solutions, we take the action to be of the form

$$
S=-\frac{1}{16 \pi} \int_{M}(R-2 \Lambda) \sqrt{-g} d^{4} x
$$

such that when solving the equations of motion, the Ricci scalar is proportional to the cosmological constant. This means that for the de Sitter solution, we have $\Lambda<0$ and for the anti-de Sitter solution, we have that $\Lambda>0$. This follows from our choice of an action with $s_{3}=-1$. While this is different from the conventions used in most research papers on (anti-)de Sitter solutions, it allows us to be consistent with previous work for solutions without a cosmological constant, which also is the main focus in the present paper.

Following these conventions through to the Euclidean action, we find that these signs appear as:

$$
S=\frac{s_{1} s_{3}}{2 \kappa_{4}^{2}} \int_{M} \sqrt{g} R d^{4} x+\frac{s_{1} s_{4}^{\prime} \epsilon}{\kappa_{4}^{2}} \int_{\partial M} \sqrt{|\gamma|} K d^{3} x,
$$

where the fourth sign $s_{4}^{\prime}$, which arises from the definition of the second fundamental form, is discussed in the next appendix. Note that $s_{4}^{\prime}$ is distinct from $s_{4}$ in [52], which is related to the spin connection. Since we only consider bosonic fields, this sign $s_{4}$ is irrelevant for us. We also do not need to fix the fifth parameter $s_{5}$ of [52], which determines the overall sign of the $\varepsilon$-tensor, because the numerical value of the $\varepsilon$-tensor is not relevant for our calculations. 


\section{B Extrinsic curvature}

A well-posed variational problem for the Einstein-Hilbert action requires the inclusion of boundary terms which 'live' on the boundary $\partial M=\Sigma$, the exception being when $M$ is closed (compact, without boundary). For spacetimes of infinite volume the boundary is defined as the limit of a family of hypersurfaces; for example, the boundary of $\mathbb{R}^{4}$ can be defined as the limit of a family of three-spheres $S_{R}^{3}$ of radius $R$, where $R \rightarrow \infty$. In such situations, the boundary terms must be added to the Einstein-Hilbert action to deal with variations of field configurations which do not fall off fast at infinity.

The boundary term involves the (trace of the) extrinsic curvature of the boundary, regarded as an embedded submanifold. In this appendix we review how the extrinsic curvature $K_{\mu \nu}$ and its trace $K$ can be computed. We use a parameter $\epsilon= \pm 1$ to encode whether the boundary $\Sigma$ is timelike, $\epsilon=-1$ or spacelike, $\epsilon=1$. We denote the outer unit normal vector field of $\Sigma$ by $n$, so that $g(n, n)=n_{\mu} n^{\mu}=\epsilon$.

The first fundamental form, $\gamma$, is constructed out of the spacetime metric $g$ and the unit normal $n$. This tensor is transversal to $\Sigma$, that is $\gamma(N, \cdot)=0$ for all vectors $N$ which are normal to $\Sigma$. When evaluated on vectors tangent to $\Sigma$, the tensor $\gamma$ agrees with the pull-back metric $\left(\iota^{*} g\right)$ on $\Sigma$ which is induced by the embedding $\iota: \Sigma \rightarrow M$. Its mixed components $\gamma_{\nu}^{\mu}=\delta_{\nu}^{\mu}-\epsilon n^{\mu} n_{\nu}$ can be used to project tensors on $M$ onto tensors on $\Sigma$. For example, the projection of a vector $X$ at a point $p \in \Sigma$ onto a vector $X_{\|}$tangent to $\Sigma$ takes the form $X^{\mu} \mapsto X_{\|}^{\mu}=\gamma_{\nu}^{\mu} X^{\nu}$ in local coordinates.

The second fundamental form $K$, or the extrinsic curvature tensor, measures the failure of a normal vector to remain normal to $\Sigma$ under parallel transport with the Levi-Civita connection $\nabla$ of $(M, g)$ along curves on $\Sigma$. To define the second fundamental form, consider the parallel transport of a normal vector $N$ along a curve $C$ on $\Sigma$. Then $\nabla_{X} N=X^{\mu} \nabla_{\mu} X^{\nu}=0$, where $X$ is the tangent vector field of $C$. If $N$ remains a normal vector field to $\Sigma$ under parallel transport on $\Sigma$, then $g(N, Y)=0$ for all points on $C$ and all tangent vectors $Y$ to $\Sigma$ along $C$. In other words we can measure the failure of $N$ remaining normal to $\Sigma$ by studying the variation

$$
X \cdot g(N, Y)=X^{\mu} \nabla_{\mu}\left(Y^{\nu} N_{\nu}\right)=N_{\nu} X^{\mu} \nabla_{\mu} Y^{\nu}
$$

of $g(N, Y)$ along $C$. To define the extrinsic curvature, we use the normal unit vector field $n$ on $\Sigma$, which we can extend around each point $p \in M$ to a unit vector field on a neighbourhood in $M$. The extrinsic curvature is defined, using the projections introduced above, by

$$
K(X, Y)=-s_{4} n_{\mu}\left(\nabla_{X_{\|}} Y_{\|}\right)^{\mu},
$$

for vector fields $X, Y$ on $M$. It can be shown that this definition is independent of how $n$ is extended away from $\Sigma$. The overall sign of $K$ is coventional. In our work, we use that $s_{4}=1$, but note that many other authors choose $s_{4}=-1$ (see for example eq. (5) in [29]). Using that $n_{\rho} Y_{\|}^{\rho}=0$ we can evaluate $K(X, Y)$ :

$$
K(X, Y)=-n_{\rho} X_{\|}^{\sigma} \nabla_{\sigma} Y_{\|}^{\rho}=X_{\|}^{\sigma} Y_{\|}^{\rho} \nabla_{\rho} n_{\sigma}=\left(\gamma_{\mu}^{\sigma} \gamma_{\nu}^{\rho} \nabla_{\rho} n_{\sigma}\right) X^{\mu} Y^{\nu}=K_{\mu \nu} X^{\mu} Y^{\nu}
$$


This leads to the expressions

$$
K_{\mu \nu}=\gamma_{\mu}^{\rho} \gamma_{\nu}^{\sigma} \nabla_{\rho} n_{\sigma}=\nabla_{\mu} n_{\nu}-\epsilon n^{\rho} n_{\mu} \nabla_{\rho} n_{\nu}=\gamma_{\mu}^{\rho} \nabla_{\rho} n_{\nu}
$$

for the extrinsic curvature, where we used $n^{\rho} \nabla_{\mu} n_{\rho}=\frac{1}{2} \nabla_{\mu}\left(n^{\rho} n_{\rho}\right)=0$. The boundary can locally be described as the level set of a function $f$. Then $N_{\mu}=\partial_{\mu} f$ is a normal vector field, and the corresponding unit normal vector field $n_{\mu}$ is hypersurface orthogonal and satisfies the Frobenius integrability condition

$$
n_{[\mu} \nabla_{\nu} n_{\rho]}=0
$$

Contracting this relation with $n^{\rho}$ it is straightforward to obtain a relation which allows to show that $K_{\mu \nu}$ is symmetric, $K_{\mu \nu}=K_{\nu \mu}$.

An alternative definition commonly used in the literature is [29]

$$
K_{\mu \nu}=\frac{1}{2} \mathcal{L}_{n} \gamma_{\mu \nu}
$$

where $\mathcal{L}_{n}$ is the Lie derivative with respect to $n$. We can verify that this agrees with our definition by writing out the Lie derivative in terms of covariant derivatives, and using $\gamma_{\mu \nu}=g_{\mu \nu}-\epsilon n_{\mu} n_{\nu}$

$$
\begin{aligned}
\frac{1}{2} \mathcal{L}_{n} \gamma_{\mu \nu} & =\frac{1}{2}\left(n^{c} \nabla_{\rho}\left(g_{\mu \nu}-\epsilon n_{\mu} n_{\nu}\right)+\left(g_{\mu \rho}-\epsilon n_{\mu} n_{\rho}\right) \nabla_{\nu} n^{\rho}+\left(g_{\rho \sigma}-\epsilon n_{\rho} n_{\nu}\right) \nabla_{\mu} n^{\rho}\right) \\
& =\frac{1}{2}\left(\nabla_{\mu} n_{\nu}+\nabla_{\nu} n_{\mu}-\epsilon n^{\rho} \nabla_{\rho}\left(n_{\mu} n_{\nu}\right)\right)=\nabla_{\mu} n_{\nu}-\epsilon n_{\mu} n^{\rho} \nabla_{\rho} n_{\nu}
\end{aligned}
$$

were we used the contracted Frobenius integrability property of $n^{\mu}$. In our calculations, we need the trace of the extrinsic curvature,

$$
K=g^{\mu \nu} K_{\mu \nu}
$$

The trace can be easily calculated from the expression

$$
\begin{aligned}
K & =g^{\mu \nu} K_{\mu \nu}=\frac{1}{2} g^{\mu \nu}\left(\nabla_{\mu} n_{\nu}+\nabla_{\nu} n_{\mu}-\epsilon n^{\rho} \nabla_{\rho}\left(n_{\mu} n_{\nu}\right)\right) \\
& =\frac{1}{2}\left(\nabla_{\mu} n^{\mu}+\nabla^{\mu} n_{\mu}-\epsilon n^{\rho} \nabla_{\rho}\left(g^{\mu \nu} n_{\mu}\right) n_{\nu}\right)=\nabla_{\mu} n^{\mu} .
\end{aligned}
$$

We have defined the first and second fundamental form as transversal tensors on $M$, that is as tensors which vanish when contracted with normal vectors to $\Sigma$. Alternatively, they can be defined as tensors on $\Sigma$, see for example [53]. The resulting expressions are related by

$$
\gamma_{m n}=\left(\iota^{*} g\right)_{m n}=e_{m}^{\mu} e_{n}^{\nu} \gamma_{\mu \nu}\left(=e_{m}^{\mu} e_{n}^{\nu} g_{\mu \nu}\right), \quad K_{m n}=e_{m}^{\mu} e_{n}^{\nu} K_{\mu \nu}
$$

where the vector fields $e_{m}=\left(e_{m}^{\mu}\right), m=1,2,3$ define an orthonormal coordinate frame on $\Sigma$. 


\section{Charges and Hodge dualisation}

The thermodynamic formalism for the STU-model makes use of an electric-magnetic duality frame where the magnetic charges $\mathcal{P}^{A}, A=1,2,3$ have been replaced by electric charges $\tilde{\mathcal{Q}}_{A}$, so that all charges excited in our solution are electric. In this appendix we give some details of the dualization procedure and derive the expressions for the charges used in the main part of this article.

To explain the idea we use a theory with a single Abelian vector field $A_{\mu}$ with field strength $F_{\mu \nu}=\partial_{\mu} A_{\nu}-\partial_{\nu} A_{\mu}$ and a curved spacetime Maxwell type action

$$
S[A]=\int\left(-\frac{1}{4 g^{2}} F_{\mu \nu} F^{\mu \nu}\right) e d^{4} x .
$$

For Maxwell theory $g$ is a constant, but to cover the case of $\mathcal{N}=2$ vector multiplets we promote $g$ to a background field which depends on the spacetime coordinates. This background coupling can be used as a proxy for the scalar field dependent couplings $\mathcal{I}_{I J}$, as we will see below. We promote the Bianchi identity $\epsilon^{\mu \nu \rho \sigma} \partial_{\nu} F_{\rho \sigma}=0$ to a field equation by introducing the Lagrange multiplier vector field $\tilde{A}_{\mu}$ :

$$
S[F, \tilde{A}]=\int\left(-\frac{1}{4 g^{2}} F_{\mu \nu} F^{\mu \nu}-\frac{1}{2} \varepsilon^{\mu \nu \rho \sigma} \partial_{\mu} \tilde{A}_{\nu} F_{\rho \sigma}\right) e d^{4} x .
$$

Note that $\varepsilon^{\mu \nu \rho \sigma}$ denotes the Levi-Civita tensor, not the permutation symbol $\epsilon^{\mu \nu \rho \sigma}=$ $e \varepsilon^{\mu \nu \rho \sigma}=\delta_{0123}^{\mu \nu \rho \sigma}$, which is a tensor density. This parametrization is convenient because the metric determinant $e$ appears as an overall factor. While variation with respect to $\tilde{A}_{\mu}$ imposes the Bianchi identity, variation with respect to $F_{\mu \nu}$ produces its algebraic equation of motion

$$
\frac{1}{g^{2}} F^{\mu \nu}=-\varepsilon^{\mu \nu \rho \sigma} \partial_{\rho} \tilde{A}_{\sigma}=-\frac{1}{2} \varepsilon^{\mu \nu \rho \sigma} \tilde{F}_{\rho \sigma},
$$

where we defined the dual field strength tensor

$$
\tilde{F}_{\rho \sigma}=\partial_{\rho} \tilde{A}_{\sigma}-\partial_{\sigma} \tilde{A}_{\rho} .
$$

Substituting this back into (C.2) we obtain the dual action

$$
S[\tilde{A}]=\int\left(-\frac{g^{2}}{4} \tilde{F}_{\mu \nu} \tilde{F}^{\mu \nu}\right) e d^{4} x=\int\left(-\frac{1}{4 \tilde{g}^{2}} \tilde{F}_{\mu \nu} \tilde{F}^{\mu \nu}\right) e d^{4} x,
$$

where we defined the dual coupling

$$
\tilde{g}=\frac{1}{g} .
$$

The relation between the dual and the orginal field strength is

$$
\tilde{F}_{\mu \nu}=\frac{1}{g^{2}} \star F_{\mu \nu}
$$

where

$$
\star F_{\mu \nu}=\frac{1}{2} \varepsilon_{\mu \nu \rho \sigma} F^{\rho \sigma}=\frac{1}{2} e \epsilon_{\mu \nu \rho \sigma} F^{\rho \sigma}
$$


is the Hodge-dual field strength. Thus the duality exchanges electric and magnetic fields and inverts the coupling. It also exchanges Euler-Lagrange equations and Bianchi identities:

$$
\begin{aligned}
\partial^{\mu}\left(\frac{e}{g^{2}} F_{\mu \nu}\right) & =0 \Leftrightarrow \epsilon^{\mu \nu \rho \sigma} \partial_{\nu} \tilde{F}_{\rho \sigma}=0, \\
\epsilon^{\mu \nu \rho \sigma} \partial_{\nu} F_{\rho \sigma} & =0 \Leftrightarrow \partial^{\mu}\left(\frac{e}{\tilde{g}^{2}} \tilde{F}_{\mu \nu}\right)=0 .
\end{aligned}
$$

Note that we can write both field equations as Bianchi identities:

$$
d \tilde{F}=0, \quad d F=0 .
$$

This tells us how to define conserved charges. In a theory with field dependent coupling $g$, the electric and magnetic charge are defined by

$$
\mathcal{Q}=\frac{1}{8 \pi} \int_{X} \tilde{F}=\frac{1}{8 \pi} \int_{X} \frac{1}{g^{2}} \star F, \quad \mathcal{P}=\frac{1}{8 \pi} \int_{X} F,
$$

where we have chosen the normalization to be same as in the main part of the paper. For Maxwell theory, where $g=$ const, the electric charge is given by integral of the Hodgedual two-form $\star F$, leading to the standard expression for the Maxwell electric charge. For point-like charges the two-surface $X$ has the topology of a sphere. For solutions with planar symmetry, we take $X$ to be a plane. The resulting integral is divergent, but defines a finite charge (density) upon formally dividing by the volume of $X$, or by compactifying $X$ into a two-torus. The equations of motions and Bianchi identities, which are valid outside charges, tell us that both $F$ and $\tilde{F}$ are closed. This allows one to deform the integration surfaces $X$ continuously, as long as one avoids moving them through the charges, which for our solutions are located at the singularities. Often it is convenient to evaluate the charges in a limit where $X$ is pushed to infinity, and this is in particular how charges are computed in the main part of this paper.

The dualization procedure can be used to replace magnetic charges by electric charges. This can be convenient since in a fixed duality frame electric charges are Noether charges and can couple minimally to the gauge field, whereas magnetic charges are topological and do not have local couplings to the gauge field. For black hole thermodynamics we find it convenient to replace magnetic charges by electric charges in the main part of the paper. The dual charges are found by replacing $F$ by $\tilde{F}$. Using that

$$
\tilde{\tilde{F}}=\frac{1}{\tilde{g}^{2}} \star \tilde{F}=\frac{1}{\tilde{g}^{2} g^{2}} \star \star F=-F,
$$

we find

$$
\begin{aligned}
& \tilde{\mathcal{Q}}=\frac{1}{8 \pi} \int_{X} \tilde{\tilde{F}}=-\frac{1}{8 \pi} \int_{X} F=-\mathcal{P}, \\
& \tilde{\mathcal{P}}=\frac{1}{8 \pi} \int_{X} \tilde{F}=\mathcal{Q} .
\end{aligned}
$$

Note that the transformation $(\mathcal{Q}, \mathcal{P}) \rightarrow(-\mathcal{P}, \mathcal{Q})$ is symplectic. 
The relation between the Einstein-Maxwell theory and the Einstein-anti-Maxwell theory where the sign of the Maxwell term has been flipped, can be interpreted as an analytic continuation of the coupling: $g \rightarrow i g, g^{-2} \rightarrow-g^{-2}$. From (C.12) it is clear that this flips the signs of electric charges, $\mathcal{Q} \rightarrow-\mathcal{Q}$. The same conclusion is reached when including electric sources and defining the electric charge using Gauss law,

$$
\mathcal{Q}=-\frac{1}{8 \pi} \int_{\Sigma} \star j
$$

where $\Sigma$ is a hypersurface such that $X=\partial \Sigma$, and where $j^{\mu}$ is the charge density. Maxwell's equations relate the gauge field to the charge density:

$$
d\left(\frac{1}{g^{2}} \star F\right)=-\star j .
$$

Integrating over a hypersurface $\Sigma$ and applying the Gauss-Stokes theorem we obtain:

$$
\mathcal{Q}=\frac{1}{8 \pi} \int_{\Sigma} d\left(\frac{1}{g^{2}} \star F\right)=\frac{1}{8 \pi} \int_{\partial \Sigma} \frac{1}{g^{2}} \star F .
$$

As we consider sources as external, the analytical continuation of the coupling $g$ changes the sign of $\mathcal{Q}$.

Electric-magnetic duality can be extended to theories with multiplet vector fields, including $\mathcal{N}=2$ vector multiplets with bosonic Lagrangian (5.1). In these theories electricmagnetic duality becomes part of a larger group of symplectic transformations, which acts continuously on gauge fields, but is broken to a discrete subgroup once charge quantization is taken into account. We refer to [54] for a general discussion. In this paper we consider the STU-model, and for our solutions we only need the consistent truncation where the coupling matrices are restricted by $\mathcal{R}_{I J}=0$. Since the remaining coupling matrix $\mathcal{I}_{I J}$ is diagonal, the vector field part of the Lagrangian (5.1) reduces to

$$
e_{4}^{-1} \mathcal{L}=+\frac{1}{4 \kappa_{4}^{2}}\left(\mathcal{I}_{00} F_{\mu \nu}^{0} F^{0 \mid \mu \nu}+\sum_{A=1}^{3} \mathcal{I}_{A A} F_{\mu \nu}^{A} F^{A \mid \mu \nu}\right)+\cdots
$$

This amounts to four copies of the type of vector field Lagrangian we have considered before. Our solution carries charges $\left(\mathcal{Q}_{0}, \mathcal{P}^{A}\right)$, which we can map to the purely electric charges $\left(\mathcal{Q}_{0}, \tilde{\mathcal{Q}}_{A}\right)$, where $\tilde{\mathcal{Q}}_{A}=-\mathcal{P}^{A}$.

For reference we bring the expressions for the charges to the form used for explicit computations in the main part of the paper:

$$
\begin{aligned}
& \mathcal{Q}_{0}=\frac{1}{8 \pi} \int_{X} \tilde{F}_{0}=\frac{1}{8 \pi} \int_{X} \star\left(-\mathcal{I}_{00} F^{0}\right), \\
& \tilde{\mathcal{Q}}_{A}=-\mathcal{P}^{A}=-\frac{1}{8 \pi} \int_{X} F^{A}=\frac{1}{8 \pi} \int_{X} \star\left(-\mathcal{I}^{A A} \tilde{F}_{A}\right) .
\end{aligned}
$$

\section{Kruskal extensions and classification of trapping horizons}

In this section, we perform Kruskal-like coordinate transformations which allow us to construct the maximal analytic extensions of all metrics considered within this paper. This 
allows us to understand their global causal structure and to identify the type of all horizons using the classification of trapping horizons reviewed in section 2.4. Solutions fall into two categories, which are distinguished by the causal relation between their interior and exterior regions. For all solutions, we call regions exterior if transverse/radial null geodesics reach a horizon in one direction, but can be extended to infinite affine parameter in the other direction. In terms of our standard transverse/radial coordinate, the asymptotic region is at $r \rightarrow \infty$. In contrast, regions are called interior regions if transverse/radial null geodesics terminate at a curvature singularity in one direction and reach a horizon in the other. We refer to solutions with a static exterior region as black hole solutions, and those with a time-dependent exteriors as cosmological solutions. When using the KodamaHayward method $[33,36]$ for computing the surface gravity, it is positive for black hole solutions, but negative for cosmological solutions.

The static line elements considered in the main part are used to define ingoing and outgoing null geodesics and to fix the global time orientation of the maximally extended spacetime. This is important since the extension contains two isometric static regions, where the line element takes same form in terms of coordinates $t, r$, but where the timelike Killing vector field $\partial_{t}$ is future-directed in one region, but past-directed in the other (where future-directed is defined globally by picking one of the patches to fix the time orientation). Starting from a 'standard static patch,' which fixes the definition of ingoing/outgoing and determines the direction of time, we define Kruskal coordinates and obtain a maximally extended spacetime containing three additional regions. By computing the expansions of null geodesic congruences for each regions, we can identify the types of the horizons separating them. For thermodynamics we consider future horizons, where the exterior region can causally influence the interior, but not vice versa. The horizons between such regions are future outer horizons for black hole solutions and future inner horizons for cosmological solutions. For the thermodynamic formalism based on the Euclidean action that we use in the main part, we assume that temperature and surface gravity are related according to $[34,35]$, that is they are proportional. Then black holes have positive temperature, while (contracting) cosmologies have negative temperature.

\section{D.1 Black hole solutions}

For the following discussion, we will be considering solutions with a line element given by

$$
d s^{2}=-f(r) d t^{2}+f(r)^{-1} d r^{2}+r^{2} d \vec{X}^{2},
$$

where $d \vec{X}^{2}=d \Omega^{2}$ for spherically symmetric solutions, and $d \vec{X}^{2}=d x^{2}+d y^{2}$ for planar symmetric solutions. The function $f(r)$ is assumed to have a simple zero at $r=r_{h}>0$, and to be positive in the exterior region $r_{h}<r<\infty,-\infty<t<\infty$. This implies that the surface gravity of the horizon is positive:

$$
\kappa=\left.\frac{1}{2} \partial_{r} f(r)\right|_{r=r_{h}}>0 .
$$

The solution is static in the exterior region, but the Killing vector field $\partial_{t}$ becomes spacelike for $r<r_{h}$. This situation is known from the event horizons of black holes. The two explicit choices for $f$ that are relevant for us are 
1. The Schwarzschild solution

$$
f(r)=1-\frac{2 M}{r}, \quad \kappa=\frac{1}{4 M} .
$$

2. The planar anti-Einstein-Maxwell solution

$$
f(r)=\frac{2 M}{r}-\frac{q^{2}}{r^{2}}, \quad \kappa=\frac{4 M^{3}}{q^{4}} .
$$

While we do not explicitly work with the Schwarzschild solution in this paper, we include it so that one may compare computations with the well-known results [32]. For the remainder of the discussion, we will keep $f(r)$ general and sometimes use its Taylor expansion near the horizon:

$$
f(r)=2 \kappa\left(r-r_{h}\right)+\mathcal{O}\left(\left(r-r_{h}\right)^{2}\right) .
$$

\section{D.1.1 Defining Kruskal coordinates}

As a first step to extending the solution beyond the horizon ${ }^{19}$ we replace the transversal/radial coordinate $r$ by the tortoise coordinate

$$
r_{\star}=\int f(r)^{-1} d r, \quad-\infty<r_{\star}<\infty .
$$

Then we can define the future-pointing null coordinates

$$
x_{+}=t+r_{\star}, \quad x_{-}=t-r_{\star}, \quad-\infty<x_{ \pm}<\infty .
$$

In these coordinates, the metric is given by

$$
d s^{2}=-f(r) d x_{+} d x_{-}+r^{2} d \vec{X}^{2},
$$

where we have used that

$$
d x_{+}=d t+f(r)^{-1} d r, \quad d x_{-}=d t-f(r)^{-1} d r .
$$

It is understood that $r$ is now a function of $x_{ \pm}$, which we only need to define implicitly,

$$
r=r\left[r_{\star}\left(x_{+}, x_{-}\right)\right] .
$$

Outgoing null congruences consist of null rays with $x_{-}=$constant and propagate in the positive $x_{+}$direction, while ingoing null congruences are defined by $x_{+}=$constant and move in the positive $x_{-}$direction.

To extend our solution, we define Kruskal coordinates by

$$
\begin{array}{cccc}
X_{-}=-e^{-x_{-} \kappa}, & -\infty<X_{-}<0 & \Leftrightarrow & -\infty<x_{-}<\infty, \\
X_{+}=+e^{x_{+} \kappa}, & 0<X_{+}<\infty & \Leftrightarrow-\infty<x_{+}<\infty .
\end{array}
$$

\footnotetext{
${ }^{19}$ It will turn out that the naive extension of the line element to $r<r_{h}$ covers part of the maximal extension. This is of course well known for standard solutions, such as Schwarzschild.
} 
$X_{-}$

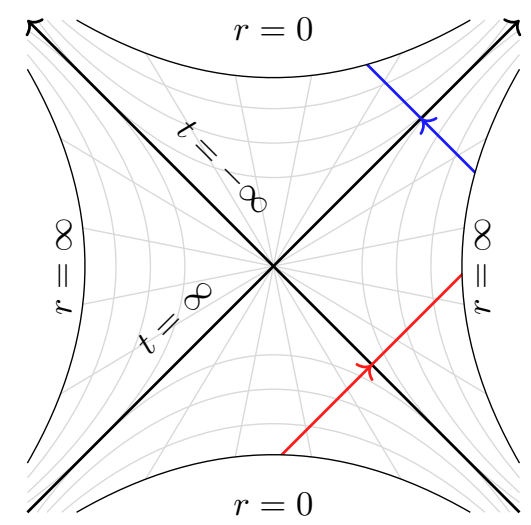

(a) Black hole solutions
$X_{-} \quad X_{+}$

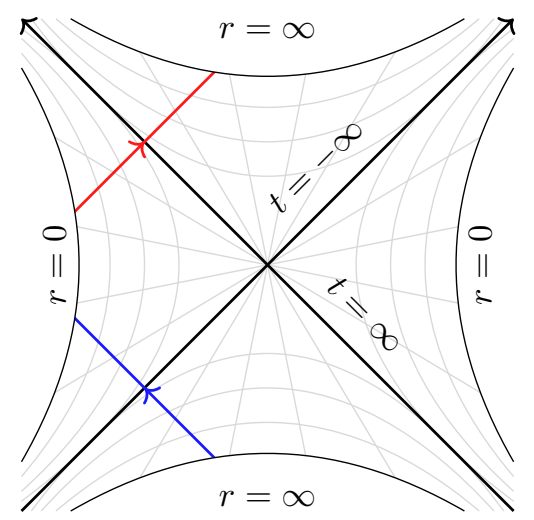

(b) Cosmological Solutions

Figure 5. Kruskal diagrams for black hole and cosmological solutions. Surfaces of constant $r$ are hyperbola and surfaces of constant $t$ are straight lines. Also included are the ingoing (blue) and outgoing (red) null geodesics which are future-pointing.

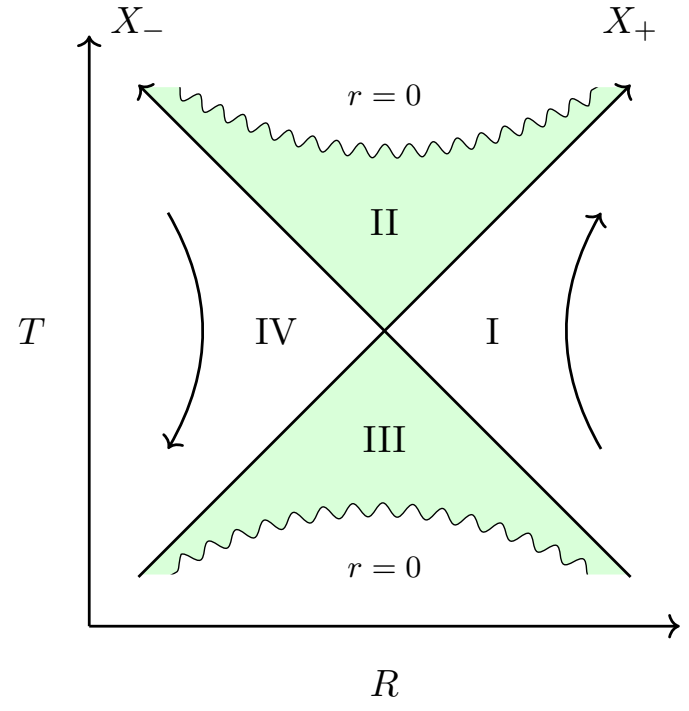

(a) Black hole solutions

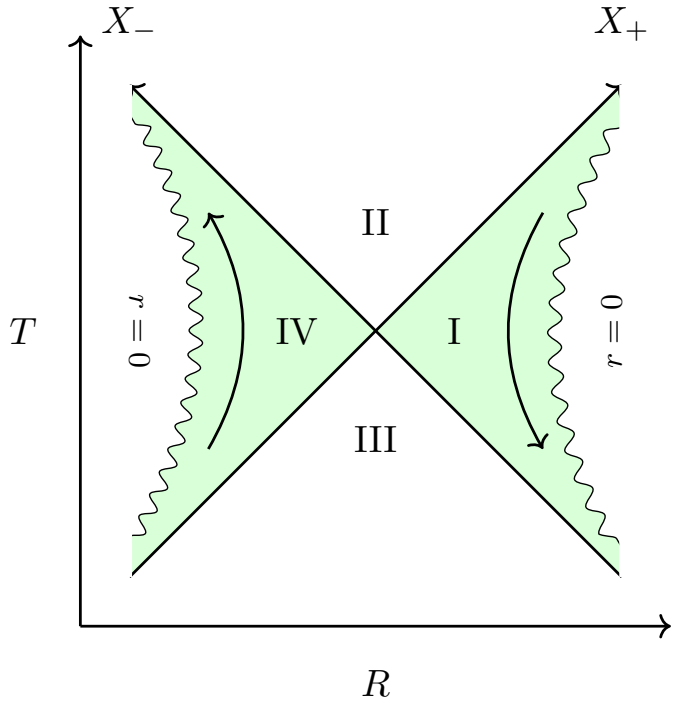

(b) Cosmological Solutions

Figure 6. Comparison of the Kruskal diagrams for black hole and cosmological solutions. Shaded regions correspond to the interior regions, curved lines show the direction of the Killing vector field in the static patches of the spacetime.

The Kruskal extension is obtained by dropping the constraints $X_{-}<0, X_{+}>0$, resulting in the four regions of figure 6 . In order for this extension to be well defined, we must make sure that the metric is non-degenerate at the horzions $r=r_{h}$, where $f(r)$ has its zero. It is for this purpose that factors of the surface gravity $\kappa$ have been included in the definition of $X_{ \pm}$. 
The line element in Kruskal coordinates is given by

$$
d s^{2}=-\frac{1}{\kappa^{2}} f(r) e^{-2 \kappa r_{\star}} d X_{+} d X_{-}+r^{2} d \vec{X}^{2},
$$

where $r\left(X_{+}, X_{-}\right)$is determined implicitly by

$$
X_{+} X_{-}=-e^{2 \kappa r_{\star}} .
$$

We can also implicitly define the coordinate $t\left(X_{+}, X_{-}\right)$from

$$
\frac{X_{+}}{X_{-}}=-e^{2 \kappa t}
$$

To show that (D.3) is regular on the horizon, we look at the Taylor expansion (D.2), and integrate to obtain $r_{\star}$,

$$
-2 \kappa r_{\star}=\int\left(-\frac{2 \kappa}{2 \kappa\left(r-r_{h}\right)}+\mathcal{O}\left(r-r_{h}\right)\right) d r=-\log \left(r-r_{h}\right)+\mathcal{O}\left(\left(r-r_{h}\right)^{2}\right) .
$$

We see that the zeros are cancelled,

$$
-f(r) e^{-2 \kappa r_{\star}}=-2 \kappa\left(r-r_{h}\right) e^{-\log \left(r-r_{h}\right)}=-2 \kappa+\mathcal{O}\left(\left(r-r_{h}\right)^{2}\right),
$$

and thus, on the horizon we find the line element

$$
d s^{2}=-\frac{2}{\kappa} d X_{+} d X_{-}+r_{h}^{2} d \Omega^{2} .
$$

As a result, one can consider the metric (D.3) with the coordinates extended to

$$
-\infty<X_{ \pm}<\infty
$$

subject to the constraint that $r\left(X_{+}, X_{-}\right)>0$. This produces the four regions of the diagram in figure 6 .

With our choice of signs in (D.3), the static region we started with is identified with region I. The Kruskal extension contains a second static region, region IV. In Region IV the null Kruskal coordinates take values $X_{-}>0$ and $X_{+}<0$. One can introduce null coordinates $x_{ \pm}$by

$$
\begin{array}{lrl}
X_{-}=+e^{-x_{-} \kappa}, & 0<X_{-}<\infty & \leftrightarrow \infty>x_{-}>-\infty . \\
X_{+}=-e^{x_{+} \kappa}, & -\infty<X_{+}<0 & \leftrightarrow \infty>x_{+}>-\infty .
\end{array}
$$

Observe that $x_{+}, x_{-}$are directed opposite to $X_{+}, X_{-}$in Region IV. If we go back from $x_{ \pm}$, to $t, r_{\star}$ and further to $t, r$, using the same relations as in Region I, the metric assumes the same local form (D.3) as in Region I. But globally, there is a difference compared to Region $\mathrm{I}$, as $t, r$ point the opposite way: $t$ downwards, $r$ leftwards. As a result, ingoing lightfronts move in positive $X_{+}=$negative $x_{+}$direction. Outgoing lightfronts move in the positive $X_{-}=$negative $x_{-}$direction. The association of $X_{-}, X_{+}$with in/out-moving lightfronts is reversed compared to Region I, see figure 7. 


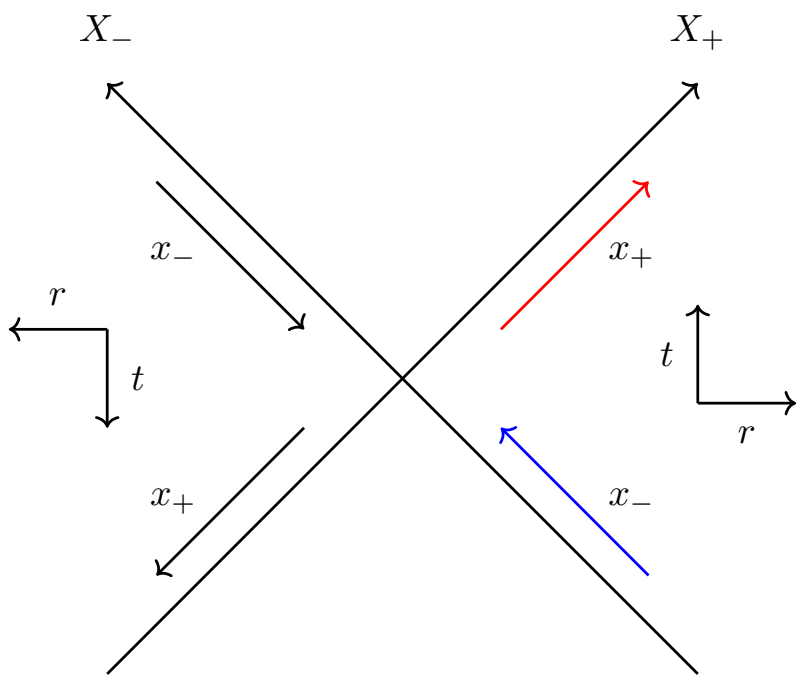

Figure 7. Flow of coordinates in the static regions of the Kruskal diagram for black hole solutions. The red arrow denotes future directed outgoing null geodesics, the blue arrow denotes future directed ingoing null geodesics.

The global spacetime is time-orientable and time-reversal symmetric. We should not conclude that time is flowing backwards in Region IV. If we choose a global time orientation that points in the same direction as $t$ in Region I, then physical time in Region IV is measured by $-t$. We refer to Region I as the 'standard' static region, and the definition of Kruskal coordinates as the 'standard embedding' of the static region into its Kruskal extension.

\section{D.1.2 Calculation of expansions}

With the global causal structure understood, we are now in a position to write down futuredirected ingoing and outgoing null geodesics and then calculate their expansions. Before this, we perform some intermediate calculations.

For $X_{ \pm}$in region $\mathrm{I}$, we can calculate $d r$ from

$$
X_{+} d X_{-}+X_{-} d X_{+}=-2 \kappa e^{2 \kappa r_{\star}} d r_{\star}=2 \kappa X_{+} X_{-} d r_{\star},
$$

which allows us to write

$$
d r=\frac{f(r)}{X_{+} X_{-}} \frac{1}{2 \kappa}\left(X_{+} d X_{-}+X_{-} d X_{+}\right) .
$$

Similarly, we can write down

$$
d t=-\frac{1}{X_{+} X_{-}} \frac{1}{2 \kappa}\left(X_{+} d X_{-}-X_{-} d X_{+}\right) .
$$

In the original coordinates, the Killing vector field is given by $\xi=\frac{\partial}{\partial t}$, and using the metric tensor we can write down the co-vector $\xi^{b}=-f(r) d t .{ }^{20}$ Using the results from above, we

\footnotetext{
${ }^{20}$ We use 'musical' notation to distinguish between vectors and the corresponding co-vectors (one-forms).
} 
can rewrite the Killing co-vector field in terms of our new Kruskal-like coordinates

$$
\xi^{b}=-f(r) d t=\frac{f(r)}{X_{+} X_{-}} \frac{1}{2 \kappa}\left(X_{+} d X_{-}-X_{-} d X_{+}\right) .
$$

The corresponding vector field is

$$
\xi=\kappa\left(-X_{-} \frac{\partial}{\partial X_{-}}+X_{+} \frac{\partial}{\partial X_{+}}\right) .
$$

To calculate the expansions we choose the following normal null co-vector fields for our two null congruences:

$$
N_{+}^{b}=-\frac{1}{\kappa} d X_{-}, \quad N_{-}^{b}=-\frac{1}{\kappa} d X_{+}
$$

or, as vector fields,

$$
N_{+}=-2 \kappa \frac{X_{+} X_{-}}{f(r)}\left(\frac{\partial}{\partial X_{+}}\right), \quad N_{-}=-2 \kappa \frac{X_{+} X_{-}}{f(r)}\left(\frac{\partial}{\partial X_{-}}\right) .
$$

The normalisation and overall sign has been set in (D.5) such that

$$
\xi \cdot N_{+}=X_{-}, \quad \xi \cdot N_{-}=-X_{+},
$$

which ensure that the normal null vectors $N_{ \pm}$are future directed, $\xi \cdot N_{ \pm}<0$, in region I where the Killing vector flows in the direction of global time. Their expansions are calculated

$$
\theta_{ \pm}=\nabla_{\mu} N_{ \pm}^{\mu}=\frac{1}{\sqrt{-g}} \partial_{\mu}\left(\sqrt{-g} N_{ \pm}^{\mu}\right), \quad \sqrt{-g}=-\frac{f(r) \sqrt{h}}{X_{+} X_{-}} \frac{r^{2}}{2 \kappa^{2}}
$$

where we use that $h=\operatorname{det}\left(d \vec{X}^{2}\right)$ for either the two-sphere or the two-plane depending on the symmetry of the solution. This results in

$$
\theta_{ \pm}=-\frac{4 \kappa}{r} \frac{X_{+} X_{-}}{f(r)} \frac{\partial r}{\partial X_{ \pm}}
$$

To calculate the sign, we use (D.4) to find that

$$
\frac{\partial r}{\partial X_{ \pm}}=\frac{f(r)}{X_{+} X_{-}} \frac{X_{\mp}}{2 \kappa}
$$

which gives the result

$$
\theta_{+}=-\frac{2 X_{-}}{r}, \quad \theta_{-}=-\frac{2 X_{+}}{r} .
$$

Thus the expansions change signs across horizons, and the resulting pattern already completely determines the nature of each horizon. For completeness, we calculate the Lie derivatives of expansions vanishing at the horizon, though we stress that their signs are completely determined by the sign change of the corresponding expansion. We find that

$$
\mathcal{L}_{N_{-}} \theta_{+}=-2 \kappa \frac{X_{+} X_{-}}{f(r)} \partial_{-}\left(-\frac{2 X_{-}}{r}\right)=\frac{4 \kappa}{r}\left(\frac{X_{+} X_{-}}{f(r)}\right)-\frac{2 X_{+} X_{-}}{r^{2}},
$$




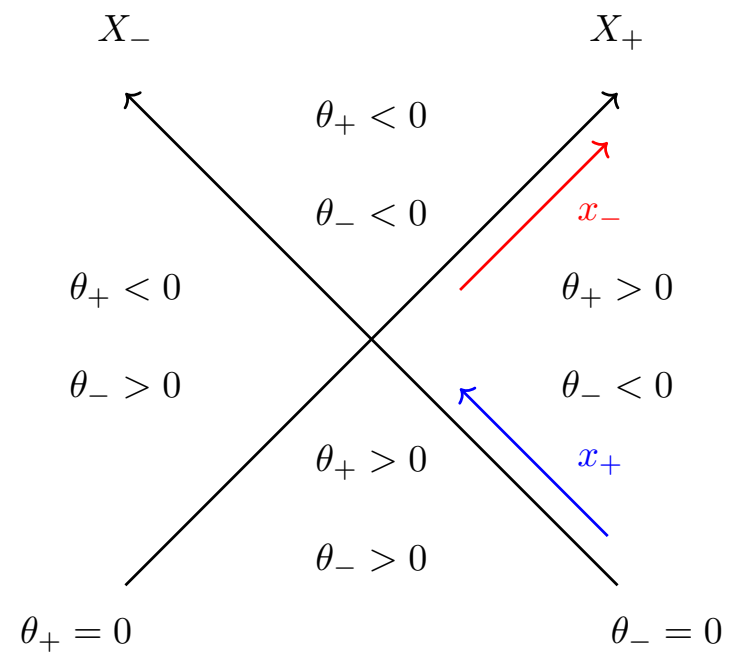

Figure 8. Signs of the expansions $\theta_{ \pm}$in the four quadrants of the Kruskal diagram for a our black hole solutions for which $\kappa>0$.

which evaluated on the horizon, gives

$$
\left.\mathcal{L}_{N_{-}} \theta_{+}\right|_{r=r_{h}}=\frac{4 \kappa}{r_{h}} \cdot\left(-\frac{1}{2 \kappa}\right)=-\frac{2}{r_{h}} .
$$

By symmetry, we can see that

$$
\left.\mathcal{L}_{N_{+}} \theta_{-}\right|_{r=r_{h}}=-\frac{2}{r_{h}}
$$

We can now look at the two horizons in region I, where we have $X_{-}<0$ and $X_{+}>0$. For the horizon given by $X_{+}=0$, we have:

$$
\theta_{+}>0, \quad \theta_{-}=0, \quad \mathcal{L}_{N_{+}} \theta_{-}<0,
$$

which is a past outer horizon. For the horizon set by $X_{-}=0$, we have that

$$
\theta_{+}=0, \quad \theta_{-}<0, \quad \mathcal{L}_{N_{-}} \theta_{+}<0,
$$

which is a future outer horizon. The expansions for all four regions are illustrated in figure 8.

Since the causal and expansion properties of the Kruskal diagram do not depend on details of the function $f$, the interpretation is the same as for the Kruskal-Schwarzschild solution, for all members of this class. The future outer horizon is the event horizon of a black hole, and the past outer horizon is the horizon for the white hole region. For thermodynamics we use the horizon where causal geodesics cross from the exterior to the interior, which is the future outer horizon between Regions I and II. This horizon has positive temperature, $T_{H} \propto \kappa>0$. 


\section{D.2 Cosmological solutions}

We now turn our attention to the cosmological solutions in this paper. The line element takes the same form

$$
d s^{2}=-f(r) d t^{2}+f(r)^{-1} d r^{2}+r^{2} d \vec{X}^{2}
$$

as before, but now $f(r)$ is positive for $r_{\text {sing }}<r<r_{h}$, where $r_{\text {sing }}$ is the position of the singularity. The metric is static in this region, so that compared to the previous class the roles of exterior and interior are exchanged, that is, the interior region is static. We assume that $f(r)$ has a simple zero and therefore changes sign at $r=r_{h}$. Since the Killing vector field $\partial_{t}$ becomes spacelike for $r>r_{h}$, the outside region is dynamical. We assume that $f(r)$ is negative for $r_{h}<r<\infty$, with $r \rightarrow \infty$ at infinite distance. Thus the horizon at $r=r_{h}$ is a cosmological horizon. Under the conditions we have imposed on $f$ the surface gravity is negative

$$
\kappa=\left.\frac{1}{2} \partial_{r} f(r)\right|_{r=r_{h}}<0 .
$$

Explicit choices for $f(r)$, which we consider in this paper, are

1. De Sitter solution

$$
f(r)=1-\frac{r^{2}}{L^{2}}, \quad \kappa=-\frac{1}{L} .
$$

2. Planar Einstein-Maxwell solution

$$
f(r)=\left(-\frac{2 M}{r}+\frac{q^{2}}{r^{2}}\right), \quad \kappa=-\frac{4 M^{3}}{q^{4}} .
$$

3. Planar STU solution

$$
f(r)=\frac{1-\alpha r}{2 \sqrt{\mathcal{H}_{0} \mathcal{H}_{1} \mathcal{H}_{2} \mathcal{H}_{3}}}, \quad \mathcal{H}_{a}=\beta_{a}+\gamma_{a} r, \quad \kappa=-\frac{\alpha^{3}}{4} \frac{\sqrt{\gamma_{0} \gamma_{1} \gamma_{2} \gamma_{3}}}{Q_{0} P^{1} P^{2} P^{3}} .
$$

For the remainder of the discussion, we will keep $f(r)$ general and sometimes use its Taylor expansion near the horizon:

$$
f(r)=2 \kappa\left(r-r_{h}\right)+\mathcal{O}\left(\left(r-r_{h}\right)^{2}\right) .
$$

\section{D.2.1 Defining Kruskal coordinates}

As in the black hole case we start by defining a Tortoise coordinate

$$
r_{\star}=\int f(r)^{-1} d r, \quad 0<r_{\star}<\infty .
$$

Then we introduce future-pointing null coordinates

$$
x_{+}=t+r_{\star}, \quad x_{-}=t-r_{\star}, \quad-\infty<x_{ \pm}<\infty, \quad r\left(x_{+}, x_{-}\right)>r_{\text {sing }} .
$$

The line element takes the form

$$
d s^{2}=-f(r) d x_{+} d x_{-}+r^{2} d \vec{X}^{2},
$$


where $r$ is an implicitly defined function of $x_{ \pm}$. Note that $x_{+}$is future- and outwardpointing while $x_{-}$is future- and inward-pointing in the interior region. This is the same assignment as in black hole solutions considered previously. With coordinates fixed in this way, we can clearly see what is the difference compared to the static patch of the black hole solutions. Since $r$ points in the opposite direction, the roles of interior and exterior are exchanged, where interior means $r<r_{h}$. While $x_{+}$points outwards in both cases, it points away form the horizon for the black hole solutions, but towards the horizon for the cosmological ones. This makes it natural to define Kruskal coordinates such that the standard static region, which we use to fix the overall time orientation, is Region IV, rather than Region I.

We start with the static line element, rewritten using null coordinates $x_{ \pm}$, where $x_{+}$ is future-pointing and outward-pointing, while $x_{-}$is future-pointing and inward-pointing, relative to the local coordinates $t, r$. This fixes the definitions of the expansion $\theta_{ \pm}$, and the direction of physical time. Next, we define global null Kruskal coordinates $X_{ \pm}$such that they point in the same direction as $x_{ \pm}$.

$$
\begin{array}{lrll}
X_{+}=-e^{\kappa x_{+}} & -\infty<X_{+}<0 & \Leftrightarrow-\infty<x_{+}<\infty, \\
X_{-}=e^{-\kappa x_{-}} & 0<X_{-}<\infty & \Leftrightarrow-\infty<x_{-}<\infty,
\end{array}
$$

where the factors of the surface gravity have been included to make manifest that the metric is regular at $r=r_{h}$ where $f(r)$ has its zero. We have also used that $\kappa<0$. The standard static patch is Region IV, and it is illustrative to compare the black hole case and the cosmological case in figures 5 and 6 .

The line element in Kruskal coordinates is given by

$$
d s^{2}=-\frac{f(r) e^{-2 \kappa r_{\star}}}{\kappa^{2}} d X_{+} d X_{-}+r^{2} d \vec{X}^{2} .
$$

We can show this is regular on the horizon using the same method as previously. By expanding at the horizon, we find

$$
-2 \kappa r_{\star}=\int\left(-\frac{2 \kappa}{2 \kappa\left(r-r_{h}\right)}+\mathcal{O}\left(r-r_{h}\right)\right) d r=-\log \left(r_{h}-r\right)+\mathcal{O}\left(\left(r-r_{h}\right)^{2}\right),
$$

where we note that the $\log \left(r_{h}-r\right)$ is different from the black hole case, as in the static patch we have $r<r_{h}$. Putting this together, we obtain

$$
-f(r) e^{-2 \kappa r_{\star}}=-2 \kappa\left(r-r_{h}\right) e^{-\log \left(r_{h}-r\right)}=2 \kappa+\mathcal{O}\left(\left(r-r_{h}\right)^{2}\right),
$$

such that on the horizon, the line element is given by

$$
d s^{2}=\frac{2}{\kappa} d X_{+} d X_{-}+r_{h}^{2} d \vec{X}^{2} .
$$

We see that we can extend the Kruskal null coordinates such that

$$
-\infty<X_{ \pm}<\infty
$$

subject to the constraint that $r\left(X_{+}, X_{-}\right)>r_{\text {sing }}$, where we implicitly write

$$
X_{+} X_{-}=-e^{2 \kappa r_{\star}}, \quad \frac{X_{+}}{X_{-}}=-e^{2 \kappa t} .
$$

The direction of various coordinates in the respective regions is shown in figure 9 . 


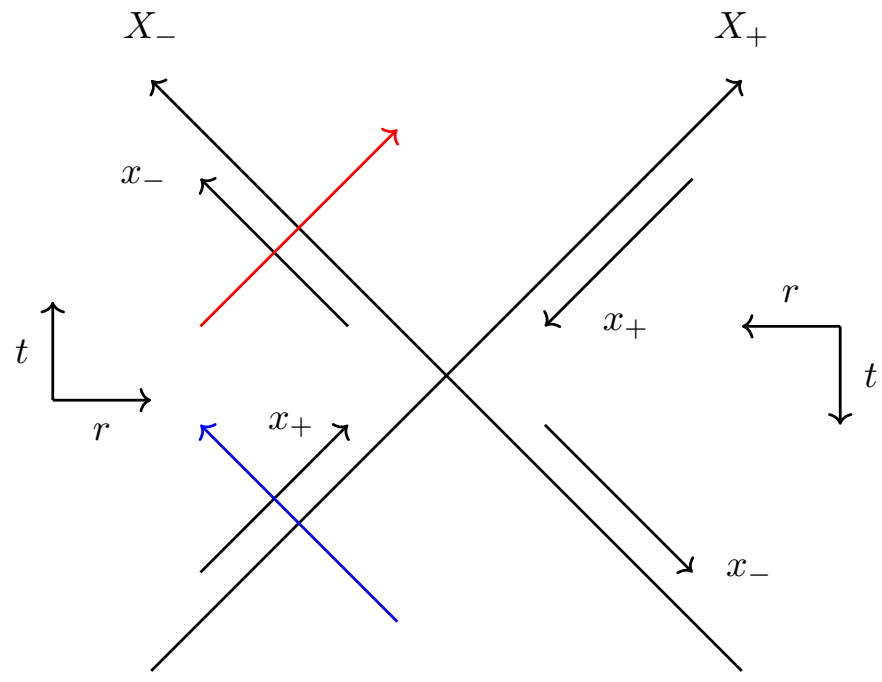

Figure 9. Flow of coordinates in the static regions of the Kruskal diagram for cosmological solutions. The red arrow denotes future directed outgoing null geodesics, the blue arrow denotes future directed ingoing null geodesics.

\section{D.2.2 Calculating the expansions}

As before, we precalculate a few useful relations for our calculations, which we find are identical to the results for the black hole solutions, namely

$$
\begin{aligned}
d r & =\frac{f(r)}{X_{+} X_{-}} \frac{1}{2 \kappa}\left(X_{+} d X_{-}+X_{-} d X_{+}\right), \\
d t & =-\frac{1}{X_{+} X_{-}} \frac{1}{2 \kappa}\left(X_{+} d X_{-}-X_{-} d X_{+}\right) .
\end{aligned}
$$

Again as in the calculation for black hole solutions, we start with the Killing vector field in our original coordinates: $\xi=\frac{\partial}{\partial t}$. Using the metric tensor write down the co-vector field $\xi^{b}=-f(r) d t$, which we can rewrite with the results above to write down the Killing co-vector field in terms of the Kruskal-like coordinates

$$
\xi^{b}=-f(r) d t=\frac{f(r)}{X_{+} X_{-}} \frac{1}{2 \kappa}\left(X_{+} d X_{-}-X_{-} d X_{+}\right),
$$

which the corresponding vector given by

$$
\xi=\kappa\left(-X_{-} \frac{\partial}{\partial X_{-}}+X_{+} \frac{\partial}{\partial X_{+}}\right) .
$$

We now write down the geodesics which are future-pointing within region IV, where the normalisation of the co-vectors is handpicked to ensure this property:

$$
N_{+}^{b}=\frac{1}{\kappa} d X_{-}, \quad N_{-}^{b}=\frac{1}{\kappa} d X_{+},
$$

or as vectors

$$
N_{+}=2 \kappa \frac{X_{+} X_{-}}{f(r)}\left(\frac{\partial}{\partial X_{+}}\right), \quad N_{-}=2 \kappa \frac{X_{+} X_{-}}{f(r)}\left(\frac{\partial}{\partial X_{-}}\right)
$$


Double checking that the normals are future-pointing, we look at the inner product of these with the Killing vector field

$$
\xi \cdot N_{+}=-X_{-}, \quad \xi \cdot N_{-}=X_{+},
$$

which we see obeys $\xi \cdot N_{ \pm}<0$ in region IV. The expansions are calculated in the same manner, and we find that the relative sign imposed in the normalisation by considering region IV, rather than region I introduces a relative sign in the expansions compared to the black hole case,

$$
\theta_{+}=\frac{2 X_{-}}{r}, \quad \theta_{-}=\frac{2 X_{+}}{r} .
$$

While this already determines the types of all horizons, we also calculate the Lie derivative at the horizon explicitly. We find that

$$
\mathcal{L}_{N_{-}} \theta_{+}=2 \kappa \frac{X_{+} X_{-}}{f(r)} \partial_{-}\left(\frac{2 X_{-}}{r}\right)=\frac{4 \kappa}{r}\left(\frac{X_{+} X_{-}}{f(r)}\right)-\frac{2 X_{+} X_{-}}{r^{2}},
$$

and on the horizon, we find

$$
\left.\mathcal{L}_{N_{-}} \theta_{+}\right|_{r=r_{h}}=\frac{4 \kappa}{r_{h}} \cdot \frac{1}{2 \kappa}=\frac{2}{r_{h}},\left.\quad \mathcal{L}_{N_{+}} \theta_{-}\right|_{r=r_{h}}=\frac{2}{r_{h}} .
$$

Let us look at the left part of the Kruskal diagram that is regions III, IV and II. The physics of the sequence III, I, IV is equivalent, but parametrized differently since $\partial_{t}$ is pastpointing in region I. In region IV of the Kruskal diagram, we have $X_{-}>0$ and $X_{+}<0$. On the horizon given by $X_{+}=0$, we have:

$$
\theta_{+}>0, \quad \theta_{-}=0, \quad \mathcal{L}_{N_{+}} \theta_{-}>0
$$

which shows that this is a past inner horizon. For the horizon set by $X_{-}=0$, we have that

$$
\theta_{+}=0, \quad \theta_{-}<0, \quad \mathcal{L}_{N_{-}} \theta_{+}>0
$$

which is a future inner horizon. The expansions for all four regions is illustrated in figure 10.

When considering future-directed causal geodesics which pass through a horizon from the exterior to the interior, we must consider the future inner horizon between region III and region IV. For a future inner horizon, we have that $T_{H} \propto \kappa<0$, which aligns with the signs of the temperatures we consider throughout this paper while employing the triple Wick rotation for our cosmological solutions. Overall the sequence III, IV (or I), II, describes a cosmic bounce, since the solution is a contracting cosmology in III and an expanding cosmology in II. We expand on the global interpretation of the cosmological solution in D.4.

\section{D.3 Eddington-Finkelstein coordinates}

For completeness we also derive expressions for advanced and retarded EddingtonFinkelstein coordinates. We start with the line element

$$
d s^{2}=-f(r) d t^{2}+f(r)^{-1} d r^{2}+r^{2} d \vec{X}^{2},
$$




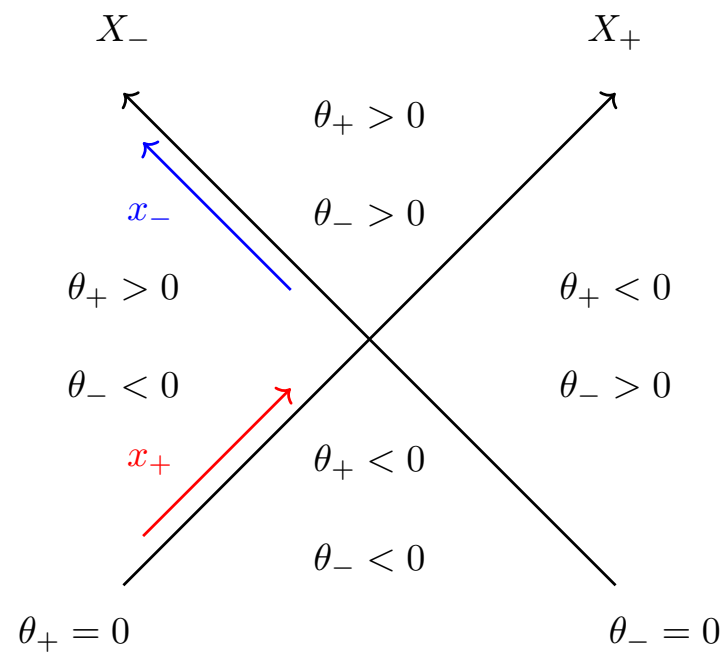

Figure 10. Signs of the expansions $\theta_{ \pm}$in the four quadrants of the Kruskal diagram for a cosmological solution where $\kappa<0$.

where we assume that there is some interval for $r$ such that $f(r)>0$, so that the line element is static, with Killing vector field $\partial_{t}$. We also assume that $f(r)$ has a simple zero at $r=r_{h}$, so that there is a second region where the Killing vector field becomes spacelike. At this point we do not specify whether the static region is $r>r_{h}$ or $r<r_{h}$, so that we can cover black holes and cosmological solutions simultaneously.

We first define advanced Eddington Finkelstein coordinates $\left(x_{+}, r, \ldots\right)$, where we omitted two further coordinates, which are $\theta, \phi$ for spherical and $x, y$ for planar symmetry. The null coordinate $x_{+}$is defined as before,

$$
d x_{+}=d t+f(r)^{-1} d r=d t+d r_{\star} .
$$

The line element takes the form

$$
d s^{2}=-f(r) d x_{+}^{2}+2 d x_{+} d r+r^{2} d \vec{X}^{2},
$$

which is manifestly regular at $r=r_{h}$, so that we can cover both the static and the non-static domain. Now we consider radial/transversal null geodesics, which must satisfy

$$
0=-f(r) d x_{+}^{2}+2 d x_{+} d r \Rightarrow d x_{+}=0 \text { or } \frac{d r}{d x^{+}}=\frac{f}{2} .
$$

Using $\lambda=x^{+}$as a curve parameter, the normal vector field for the second null congruence is

$$
U_{+}=\left(\frac{d x^{\mu}}{d \lambda}\right)=\left(U_{+}^{+}, U_{+}^{r}, \ldots\right)=\left(1, \frac{f}{2}, \ldots\right) .
$$

The corresponding co-vector has the form ${ }^{21}$

$$
U_{+}^{b}=\left(U_{+\mid+}, U_{+\mid r}, \ldots\right)=\left(-\frac{f}{2}, 1,0,0\right) .
$$

\footnotetext{
${ }^{21}$ We note here that the normal vector fields $U_{ \pm}$differ in their overall normalisation from $N_{ \pm}$defined in the previous section (D.6). The normalisation has been chosen to avoid carrying around an irrelevant numerical factor.
} 
The normal vector field $U_{-}$for the congruence $d x^{+}=0$ is a constant vector field. We normalize it such that $U_{+} \cdot U_{-}=-2$ :

$$
U_{-}=\left(U_{-}^{+}, U_{-}^{r}, \ldots\right)=(0,-2, \ldots), \quad U_{-}^{b}=\left(U_{-\mid+}, U_{-\mid r}, \ldots\right)=(-2,0, \ldots) .
$$

Both $U_{ \pm}$can be checked to be future-pointing. We could now proceed and compute the expansions $\theta_{ \pm}$but one nice feature of Eddington-Finkelstein coordinates is that they allow one to 'visualize' the expansion properties of geodesics, and the causal structure. To do this we introduce the advanced time coordinate

$$
\bar{t}:=x_{+}-r=t+r_{\star}-r \Leftrightarrow x_{+}=\bar{t}+r=t+r_{\star},
$$

and transform the null congruences $U_{ \pm}$from coordinates $\left(x_{+}, r, \ldots\right)$ to coordinates $\left.\bar{t}, r, \ldots\right)$. The result is

$$
\begin{aligned}
& \left(U_{+}^{\bar{t}}, U_{+}^{r}, \ldots\right)=\left(1-\frac{f}{2}, \frac{f}{2}, \ldots\right), \\
& \left(U_{-}^{\bar{t}}, U_{-}^{r}, \ldots\right)=(2,-2, \ldots) .
\end{aligned}
$$

Now it is manifest that $U_{-}$is future and inward-pointing for all $r$, while $U_{+}$is futurepointing, but will switch between outward-pointing and inward-pointing at $r=r_{h}$. Further details depend on whether $f$ is positive for $r>r_{h}$ or for $r<r_{h}$. Since the black hole case $r>r_{h}$ is familiar, we focus on the cosmological case, $r<r_{h}$. Then $U_{+}$is pointing outwards on the inside $r<r_{h}$, but pointing inwards on the outside $r>r_{h}$. At the horizon, $r=r_{h}$, $U_{+}$points 'upwards', that is light rays are stuck at the horizon. Drawing the lightcones associated with $U_{ \pm}$we see that future-pointing null and timelike geodesics can cross from the outside to the inside, but not the other way. This shows that $r<r_{h}$ corresponds to the standard static region IV in the cosmological Kruskal diagram, while $r>r_{h}$ corresponds to region III (and not to region II). In particular, the limit $r \rightarrow \infty$ corresponds to past timelike infinity and the coordinate $r$ is timelike and past-pointing for $r>r_{h}$.

Outside the horizons $r=r_{h}$ we can use the expression (D.7) in all regions of the Kruskal diagram. In the main part of the paper our convention is to relabel coordinates $r \leftrightarrow t$ in non-static patches, so that the timelike coordinate is always denoted $t$. We also multiply $f$ by a minus sign in non-static regions, so that $f$ remains positive. Let us here be more explicit and define $\tilde{f}(x)=-f(x)$. If we extend our solution from the static region IV to the non-static region III, then $t \rightarrow \infty$ corresponds to past timelike infinity, and $t$ is past-pointing. If we prefer to use a time coordinate in region III which is future pointing, we should relabel $t \rightarrow-t$, so that the line element in region III is ${ }^{22}$

$$
d s^{2}=-\frac{d t^{2}}{\tilde{f}(-t)}+\tilde{f}(-t) d r^{2}+t^{2} d \vec{X}^{2},-\infty<t<-r_{h} .
$$

Let us now briefly discuss retarded Eddington-Finkelstein coordinates $\left(x_{-}, r, \ldots\right)$, where, in the static patch, we replace $t$ by the ingoing future-pointing null coordinate $x_{-}$,

$$
d x_{-}=d t-f(r)^{-1} d r=d t-d r_{\star} .
$$

\footnotetext{
${ }^{22}$ Note that $f$ and $\tilde{f}$ are, in general, not even functions.
} 
The line element is

$$
d s^{2}=-f(r) d x_{-}^{2}-2 d x_{-} d r+r^{2} d \vec{X}^{2}
$$

with radial/transversal null geodesics

$$
d x_{-}=0, \quad \frac{d r}{d x_{-}}=-\frac{1}{2} f(r) .
$$

The future-pointing null normal vector fields of the null congruences are

$$
\begin{aligned}
& U_{+}=\left(U_{+}^{-}, U_{+}^{r}, \ldots\right)=(0,2, \ldots), \\
& U_{-}=\left(U_{-}^{-}, U_{-}^{r}, \ldots\right)=\left(1,-\frac{f}{2}, \ldots\right),
\end{aligned}
$$

which satisfy $U_{+} \cdot U_{-}=-2$. Introducing the new 'retarded' time coordinate $\bar{t}=x_{-}+r$, and transforming from coordinates $\left(x_{-}, r, \ldots\right)$ to coordinates $(\bar{t}, r, \ldots)$ they transform into

$$
\begin{aligned}
& U_{+}=\left(U_{+}^{\bar{t}}, U_{+}^{r}, \ldots\right)=(2,2, \ldots), \\
& U_{-}=\left(U_{-}^{\bar{t}}, U_{-}^{r}, \ldots\right)=\left(1-\frac{f}{2},-\frac{f}{2}, \ldots\right) .
\end{aligned}
$$

This shows that $U_{+}$is future and outward-pointing for all $r$ while $U_{-}$is future-pointing but changes between pointing inwards and pointing outwards at $r=r_{h}$. For black holes, where $f>0$ for $r>r_{h}$, this provides the extension into the white hole part of the Kruskal extension (region III). For cosmological solutions we see that $U_{-}$is pointing inwards for $r<r_{h}$, which we identify with the standard static region IV, and outwards for $r>r_{h}$. Future-pointing null and timelike geodesics can only cross from the inside to the outside, which shows that the outside region is region II in the cosmological Kruskal diagram. Relabeling coordinates $r \leftrightarrow t$, the metric in region II is

$$
d s^{2}=-\frac{d t^{2}}{\tilde{f}(t)}+\tilde{f}(t) d r^{2}+t^{2} d \vec{X}^{2}, \quad r_{h}<t<\infty,
$$

where $t$ is a future-pointing timelike coordinate, and where $t \rightarrow \infty$ corresponds to future timelike infinity. Comparing to (D.15) we see that the regions II and III are related by time reflection.

\section{D.4 Asymptotic limits of planar Reissner-Nordström-like solutions}

Evaluating (D.23) for the planar Reissner-Nordström solution in the asymptotic limit $t \rightarrow$ $\infty$ we obtain

$$
d s_{I I,(+\infty)}^{2}=-\frac{t}{2 M} d t^{2}+\frac{2 M}{t} d r^{2}+t^{2} d \vec{X}^{2},
$$

which is the 'positive mass' version of the planar (type D) A-III vacuum solution of pure Einstein gravity. ${ }^{23}$ Introducing a new time coordinate $t \propto \tau^{2 / 3}$ and absorbing numerical factors by rescaling $r, x, y$ this becomes

$$
d s_{I I,(+\infty)}^{2}=-d \tau^{2}+\tau^{-2 / 3} d r^{2}+\tau^{4 / 3} d \vec{X}^{2},
$$

\footnotetext{
${ }^{23}$ See [55] for background and original literature.
} 
which belongs to the class of type D Kasner solutions. These are the simplest homogeneous but anisotropic vacuum cosmological solution of pure Einstein gravity. The A-III/Kasner solution is defined for $0<t, \tau<\infty$ and describes a universe starting in a big bang at $t=\tau=0$ and then expanding in the $(x, y)$-directions while contracting in the transverse direction $r$. Its time-reversed version, which is the asymptotic solution for Region III,

$$
\begin{array}{rlr}
d s_{I I I,(-\infty)}^{2} & =\frac{t}{2 M} d t^{2}-\frac{2 M}{t} d r^{2}+t^{2} d \vec{X}^{2}, & -\infty<t<0, \\
& =-d \tau^{2}+\tau^{-2 / 3} d r^{2}+\tau^{4 / 3} d \vec{X}^{2}, & -\infty<\tau<0,
\end{array}
$$

describes a universe which contracts in the $(x, y)$ directions, expands transversally, and end in a big crunch at $t=\tau=0$. The planar Reissner-Nordström solution that has been obtained by adding non-trivial Maxwell fields describes a bouncing cosmology which interpolates between a contracting and an expanding Kasner cosmology. This removes the spacelike big crunch and big bang singularities at $t=\tau=0$ and replaces them by an intermediate region containing two timelike singularities which are shielded behind event horizons. These singularities can be interpreted as sources, and by embedding Einstein-Maxwell theory into the STU-supergravity and subsequently into string theory, these sources can be identified as certain brane configurations [24].

The asymptotic solution in Regions I and IV for the planar solution of the Einsteinanti-Maxwell theory is the 'negative mass', static version of the planar (type D) A-III vacuum solution of pure Einstein gravity, which was first described by Taub [45]. It was interpreted as the geometry outside an infinite static plane or domain wall, but the observation that neutral particles are repelled indicated a negative mass and made the physical interpretation problematic. In the planar solution to the Einstein-anti-Maxwell theory the naked timelike singularity is replaced by a spacelike singularity shielded by a horizon, so that the deformed solution describes a planar black hole. The negative mass issue becomes clearer in this description, since it is related to the negative kinetic energy of the Maxwell field in this flipped sign version of Einstein-Maxwell theory. It is an interesting question whether the embedding of this theory into type-II* string theory will allow one to give a viable physical interpretation of this solution.

\section{E Grand canonical ensemble (with fixed volume)}

In textbook thermodynamics, the internal energy $E$ (often denoted $U$ ) in the grand canonical ensemble depends on the extensive variables entropy $S$, volume $V$ and particle number $N$. In relativistic thermodynamics the particle number is not conserved, and therefore it is replaced by conserved charges. Let us consider the case of a single conserved charge $\mathcal{Q}$. We take the volume (which in black hole thermodynamics corresponds to angular momentum, or for planar solutions, linear momentum) to be fixed, so that the internal energy only depends on entropy and charge, $E=E(S, \mathcal{Q})$. The free energy $F(T, \mathcal{Q})=E-T S$ and the grand potential $\Omega(T, \mu)=E-T S-\mu \mathcal{Q}$ are related to $E(S, \mathcal{Q})$ by Legendre transformations which exchange the extensive variables $S, \mathcal{Q}$ with the intensive variables temperature $T=1 / \beta$ and chemical potential $\mu$. Various partial derivatives can be read off from the 
total differentials

$$
d E=T d S+\mu d \mathcal{Q}, d F=-S d T+\mu d \mathcal{Q}, d \Omega=-S d T-\mathcal{Q} d \mu .
$$

In particular, we obtain the following relations used in the main text:

$$
\mathcal{Q}=-\frac{\partial \Omega}{\partial \mu}, \mu=\frac{\partial F}{\partial \mathcal{Q}}, \quad \beta=\frac{1}{T}=\frac{\partial S}{\partial E}, \quad S=-\frac{\partial F}{\partial T}=\beta^{2} \frac{\partial F}{\partial \beta}
$$

and

$$
\frac{\partial(\beta F)}{\partial \beta}=F+T S=E .
$$

Open Access. This article is distributed under the terms of the Creative Commons Attribution License (CC-BY 4.0), which permits any use, distribution and reproduction in any medium, provided the original author(s) and source are credited.

\section{References}

[1] J.M. Bardeen, B. Carter and S.W. Hawking, The Four laws of black hole mechanics, Commun. Math. Phys. 31 (1973) 161 [inSPIRE].

[2] J.D. Bekenstein, Black holes and entropy, Phys. Rev. D 7 (1973) 2333 [InSPIRE].

[3] S.W. Hawking, Particle Creation by Black Holes, Commun. Math. Phys. 43 (1975) 199 [Erratum ibid. 46 (1976) 206] [INSPIRE].

[4] G.W. Gibbons and S.W. Hawking, Action Integrals and Partition Functions in Quantum Gravity, Phys. Rev. D 15 (1977) 2752 [INSPIRE].

[5] G.W. Gibbons and S.W. Hawking, Cosmological Event Horizons, Thermodynamics, and Particle Creation, Phys. Rev. D 15 (1977) 2738 [INSPIRE].

[6] R.L. Arnowitt, S. Deser and C.W. Misner, Dynamical Structure and Definition of Energy in General Relativity, Phys. Rev. 116 (1959) 1322 [INSPIRE].

[7] A. Ashtekar and A. Magnon-Ashtekar, On conserved quantities in general relativity, J. Math. Phys. 20 (1979) 793.

[8] J.D. Brown and J.W. York, Jr., Quasilocal energy and conserved charges derived from the gravitational action, Phys. Rev. D 47 (1993) 1407 [gr-qc/9209012] [INSPIRE].

[9] R.M. Wald, Black hole entropy is the Noether charge, Phys. Rev. D 48 (1993) R3427 [gr-qc/9307038] [INSPIRE].

[10] V. Iyer and R.M. Wald, Some properties of Noether charge and a proposal for dynamical black hole entropy, Phys. Rev. D 50 (1994) 846 [gr-qc/9403028] [INSPIRE].

[11] I. Papadimitriou and K. Skenderis, Thermodynamics of asymptotically locally AdS spacetimes, JHEP 08 (2005) 004 [hep-th/0505190] [INSPIRE].

[12] O.S. An, M. Cvetič and I. Papadimitriou, Black hole thermodynamics from a variational principle: Asymptotically conical backgrounds, JHEP 03 (2016) 086 [arXiv:1602.01508] [INSPIRE].

[13] A. Anabalón, N. Deruelle and F.-L. Julié, Einstein-Katz action, variational principle, Noether charges and the thermodynamics of AdS-black holes, JHEP 08 (2016) 049 [arXiv: 1606.05849] [INSPIRE]. 
[14] M. Cárdenas, O. Fuentealba and J. Matulich, On conserved charges and thermodynamics of the $A d S_{4}$ dyonic black hole, JHEP 05 (2016) 001 [arXiv:1603.03760] [INSPIRE].

[15] D. Cassani and L. Papini, The BPS limit of rotating AdS black hole thermodynamics, JHEP 09 (2019) 079 [arXiv:1906.10148] [INSPIRE].

[16] A. Ashtekar, S. Fairhurst and B. Krishnan, Isolated horizons: Hamiltonian evolution and the first law, Phys. Rev. D 62 (2000) 104025 [gr-qc/0005083] [INSPIRE].

[17] J. Maldacena, D. Stanford and Z. Yang, Conformal symmetry and its breaking in two dimensional Nearly Anti-de-Sitter space, PTEP 2016 (2016) 12C104 [arXiv:1606.01857] [INSPIRE].

[18] A. Almheiri and B. Kang, Conformal Symmetry Breaking and Thermodynamics of Near-Extremal Black Holes, JHEP 10 (2016) 052 [arXiv:1606.04108] [INSPIRE].

[19] M. Cvetič and I. Papadimitriou, AdS $S_{2}$ holographic dictionary, JHEP 12 (2016) 008 [Erratum ibid. 01 (2017) 120] [arXiv: 1608.07018] [INSPIRE].

[20] A. Gaikwad, L.K. Joshi, G. Mandal and S.R. Wadia, Holographic dual to charged SYK from $3 D$ Gravity and Chern-Simons, JHEP 02 (2020) 033 [arXiv: 1802.07746] [INSPIRE].

[21] P. Nayak, A. Shukla, R.M. Soni, S.P. Trivedi and V. Vishal, On the Dynamics of Near-Extremal Black Holes, JHEP 09 (2018) 048 [arXiv: 1802.09547] [INSPIRE].

[22] L. Li, On Thermodynamics of AdS Black Holes with Scalar Hair, Phys. Lett. B 815 (2021) 136123 [arXiv:2008.05597] [INSPIRE].

[23] R. Ruzziconi and C. Zwikel, Conservation and Integrability in Lower-Dimensional Gravity, arXiv:2012.03961 [INSPIRE].

[24] J. Gutowski, T. Mohaupt and G. Pope, From static to cosmological solutions of $\mathcal{N}=2$ supergravity, JHEP 08 (2019) 172 [arXiv: 1905.09167] [INSPIRE].

[25] D. Errington, T. Mohaupt and O. Vaughan, Non-extremal black hole solutions from the c-map, JHEP 05 (2015) 052 [arXiv: 1408.0923] [INSPIRE].

[26] C.M. Hull, Timelike $T$ duality, de Sitter space, large $N$ gauge theories and topological field theory, JHEP 07 (1998) 021 [hep-th/9806146] [INSPIRE].

[27] V. Cortés, L. Gall and T. Mohaupt, Four-dimensional vector multiplets in arbitrary signature (I), Int. J. Geom. Meth. Mod. Phys. 17 (2020) 2050150 [arXiv: 1907.12067] [InSPIRE].

[28] S.W. Hawking and S.F. Ross, Duality between electric and magnetic black holes, Phys. Rev. D 52 (1995) 5865 [hep-th/9504019] [INSPIRE].

[29] J. York, Boundary terms in the action principles of general relativity, Found. Phys. 16 (1986) 249 [INSPIRE].

[30] J.W. York, Jr., Role of conformal three geometry in the dynamics of gravitation, Phys. Rev. Lett. 28 (1972) 1082 [INSPIRE].

[31] M.K. Parikh and F. Wilczek, Hawking radiation as tunneling, Phys. Rev. Lett. 85 (2000) 5042 [hep-th/9907001] [INSPIRE].

[32] R.M. Wald, General relativity, Chicago University Press, Chicago U.S.A. (1984).

[33] S.A. Hayward, Unified first law of black hole dynamics and relativistic thermodynamics, Class. Quant. Grav. 15 (1998) 3147 [gr-qc/9710089] [InSPIRE].

[34] P. Binétruy and A. Helou, The Apparent Universe, Class. Quant. Grav. 32 (2015) 205006 [arXiv: 1406.1658] [INSPIRE]. 
[35] A. Helou, Dynamics of the four kinds of Trapping Horizons and Existence of Hawking Radiation, arXiv: 1505.07371 [INSPIRE].

[36] H. Kodama, Conserved Energy Flux for the Spherically Symmetric System and the Back Reaction Problem in the Black Hole Evaporation, Prog. Theor. Phys. 63 (1980) 1217 [INSPIRE].

[37] N.F. Ramsey, Thermodynamics and Statistical Mechanics at Negative Absolute Temperatures, Phys. Rev. 103 (1956) 20 [INSPIRE].

[38] M. Spradlin, A. Strominger and A. Volovich, Les Houches lectures on de Sitter space, in Les Houches Summer School: Session 76: Euro Summer School on Unity of Fundamental Physics: Gravity, Gauge Theory and Strings, 10, 2001 [hep-th/0110007] [INSPIRE].

[39] P. Dempster, D. Errington, J. Gutowski and T. Mohaupt, Five-dimensional Nernst branes from special geometry, JHEP 11 (2016) 114 [arXiv: 1609.05062] [INSPIRE].

[40] S.A. Hartnoll, Lectures on holographic methods for condensed matter physics, Class. Quant. Grav. 26 (2009) 224002 [arXiv:0903.3246] [INSPIRE].

[41] T. Mohaupt, Black hole entropy, special geometry and strings, Fortsch. Phys. 49 (2001) 3 [hep-th/0007195] [INSPIRE].

[42] P. Dempster, D. Errington and T. Mohaupt, Nernst branes from special geometry, JHEP 05 (2015) 079 [arXiv: 1501.07863] [INSPIRE].

[43] W.A. Sabra, Phantom Metrics With Killing Spinors, Phys. Lett. B 750 (2015) 237 [arXiv: 1507.04597] [INSPIRE].

[44] M. Bu Taam and W.A. Sabra, Phantom space-times in fake supergravity, Phys. Lett. B 751 (2015) 297 [arXiv: 1507.07721] [INSPIRE].

[45] A.H. Taub, Empty space-times admitting a three parameter group of motions, Annals Math. 53 (1951) 472.

[46] W.A. Sabra, Special geometry and space-time signature, Phys. Lett. B 773 (2017) 191 [arXiv: 1706.05162] [INSPIRE].

[47] M. Médevielle, T. Mohaupt and G. Pope, Timelike T-duality for type-II/II* compactifications on Calabi-Yau threefolds and the black hole/cosmology correspondence, work in progress.

[48] C.M. Hull, Duality and the signature of space-time, JHEP 11 (1998) 017 [hep-th/9807127] [INSPIRE].

[49] D. Klemm and M. Nozawa, Geometry of Killing spinors in neutral signature, Class. Quant. Grav. 32 (2015) 185012 [arXiv: 1504.02710] [INSPIRE].

[50] J.B. Gutowski and W.A. Sabra, Real Killing Spinors in Neutral Signature, JHEP 11 (2019) 173 [arXiv: 1905.01910] [INSPIRE].

[51] W.A. Sabra, Kasner Branes with Arbitrary Signature, Phys. Lett. B 809 (2020) 135694 [arXiv: 2005. 03953] [INSPIRE].

[52] D.Z. Freedman and A. Van Proeyen, Supergravity, Cambridge University Press, Cambridge U.K. (2012).

[53] E. Poisson, A Relativist's Toolkit. Cambridge University Press, Cambridge U.K. (2004).

[54] G.L. Cardoso and T. Mohaupt, Special geometry, Hessian structures and applications, Phys. Rept. 855 (2020) 1 [arXiv: 1909.06240] [INSPIRE].

[55] J.B. Griffiths and J. Podolsky, Exact Space-Times in Einstein's General Relativity, Cambridge University Press, Cambridge U.K. (2009). 